TEMPERATURE INSENSITIVE FIBER OPTIC PRESSURE SENSOR WITH A PI-PHASE SHIFTED FBG ON MICROSTRUCTURED FIBER

\author{
by \\ Mohammad Hossein Mahlooji \\ Master of Applied Science \\ K. N. Toosi University of Technology, 2004 \\ Bachelor of Applied Science \\ Shiraz University, 2001
}

\begin{abstract}
A thesis presented to Ryerson University
in partial fulfillment of the

requirements for the degree of

Master of Applied Science

in the program of

Electrical and Computer Engineering
\end{abstract}

Toronto, Ontario, Canada, 2018

(C) Mohammad Hossein Mahlooji, 2018 


\section{Author's Declaration}

I hereby declare that I am the sole author of this thesis. This is a true copy of the thesis, including any required final revisions, as accepted by my examiners.

I authorize Ryerson University to lend this thesis to other institutions or individuals for the purpose of scholarly research.

I further authorize Ryerson University to reproduce this thesis by photocopying or by other means, in total or in part, at the request of other institutions or individuals for the purpose of scholarly research.

I understand that my thesis may be made electronically available to the public. 


\title{
Abstract \\ TEMPERATURE INSENSITIVE FIBER OPTIC PRESSURE SENSOR WITH A PI-PHASE SHIFTED FBG ON MICROSTRUCTURED FIBER
}

\author{
Mohammad Hossein Mahlooji \\ Ryerson University - Electrical and Computer Engineering \\ Master of Applied Science - 2016
}

Fiber optic sensing technology has become a competitive device for strain measurement in many applications such as structural health monitoring and machine condition monitoring. Such success is achieved due to its advantages such as lightweight, electrically non-conductive, electromagnetic field and harsh environment immune, relatively high sensitive to strain change and the compatibility with wavelength division multiplexing method to measure many points from just one fiber cable. However, the use of fiber optic sensors in pressure measurement in gas and fluid media encounters some challenges such as temperature cross-talk to the pressure measurement, low sensitivity, and slow response in gas medium. In this work, I demonstrate both analytically and by experiments that an fiber Bragg grating (FBG) pressure sensor, inscribed in a microstructured fiber with two side holes in its cladding, can be used to measure pressure and temperature simultaneously to remove the temperature effect on measurements. The sensor has a $\boldsymbol{\pi}$-phase shifted FBG which intrinsically has a much narrower linewidth than that of the conventional FBGs and can significantly improve the sensitivity of the pressure measurement. The microstructured fiber has two different refractive indices along their two principal axes caused by its birefringence. Two FBG peaks in the measured spectra related to two principal axes change with different rates when the pressure and/or temperature is applied which makes it possible to measure the change of pressure and temperature. My results also show that the sensor responds to the pressure change instantaneously if the separation of two FBG polarization peaks is used as the measurand, which makes it suitable for dynamic pressure measurements. In addition, the sensor uses the FBG only with no transducer required and its size is extremely small, only one to two centimeter long and with a diameter of about 0.3 millimetre. 


\section{Acknowledgements}

First and above all, I praise God, the almighty for everything he has granted me.

I wish to offer my sincere thanks to several people who have assisted and guided me throughout my journey as a Master of Applied Science student. I would like to express my special appreciation and thanks to my supervisor Professor Xijia Gu for his continuous support, his patience, motivation and immense knowledge. Dr. Gu gave me the opportunity to pursue my dreams and ambitions in the field of electrical engineering. Also, I would like to extend my appreciation to my committee members, Dr. Kaamran Raahemifar and Dr. Hua $\mathrm{Lu}$ for their insightful comments and encouragement.

In particular, I would like to thank Dr. Hua Lu for providing me access to his mechanical engineering lab facilities such as temperature chamber and high pressure gas cylinder.

My time at Ryerson was made enjoyable in large part due to the many friends that became a part of my life. I would like to thank my lab mate Dr. Pan Xu for the friendly environment he provided at the lab. I am also thankful to Jiang Li for providing Fiber Optic Sensors I needed for my experiments. 


\section{Table of Contents}

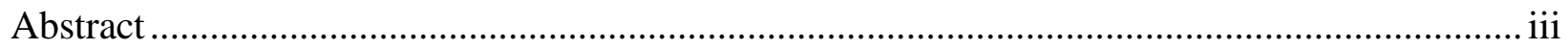

List of Abbreviations ......................................................................................................... vii

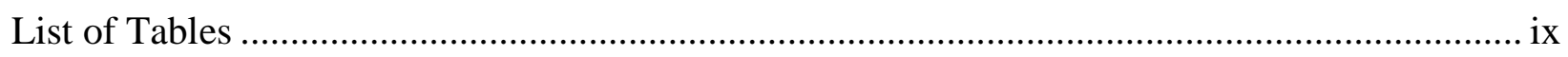

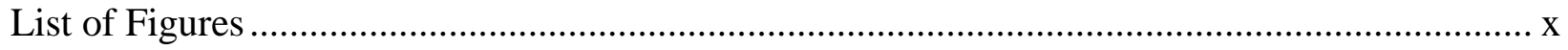

1 Introduction ........................................................................................................................................... 1

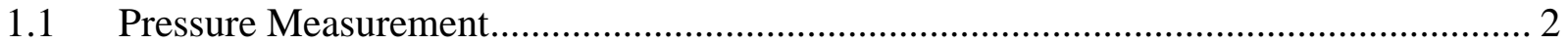

1.1.1 Mechanical Pressure Sensors .................................................................... 2

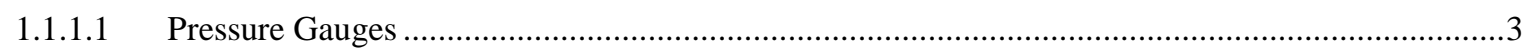

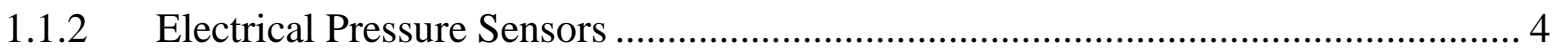

1.1.2.1 Diaphragm Pressure Transducer (Diaphragm Strain Gauges) …………………………………..

1.1.2.2 Piezoelectric Pressure Sensors ...........................................................................................................6

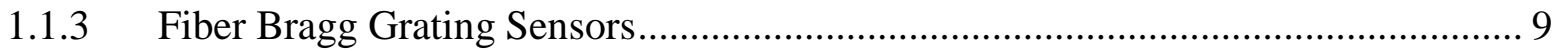

1.1.3.1 Fundamentals of Fiber Grating Sensors ................................................................................

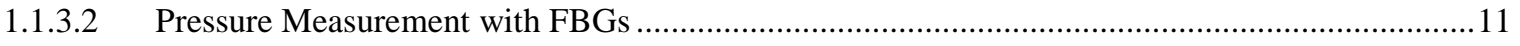

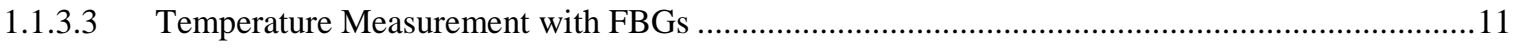

1.1.3.4 Multiplexing Measurement Using WDM Technology ………………………………………...12

$1.2 \quad$ A Review of The FBG Pressure Sensing …………............................................. 13

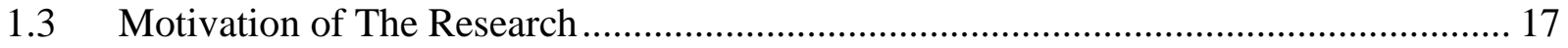

2 Simultaneous Multi-Parameter Measurement ........................................................................... 18

2.1 Multi-Parameter Measurement Applications ……………......................................... 18

2.2 Multi-Parameter Measurement Methods................................................................... 18

2.2.1 Compensation Method ...................................................................................... 19

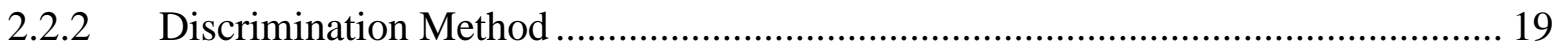

3 Finite Element Analysis Simulation of FBG …............................................................. 23

3.1 Finite Element Method Principles................................................................................ 23

3.2 FBGs Mechanical Behavior ........................................................................... 25

3.3 ANSYS and COMSOL Simulation Results ……………........................................ 28 
3.3.1 Force-Displacement Relationship of FBG Under Pressure ............................... 32

3.3.2 Effect of Fiber Optic Cross Sectional Dimension and Setup Configuration .......... 38

4 Pressure and Temperature Measurement Experiment ................................................... 43

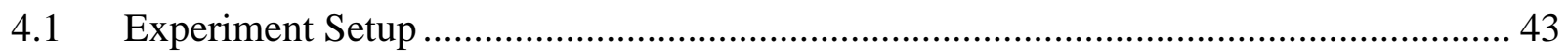

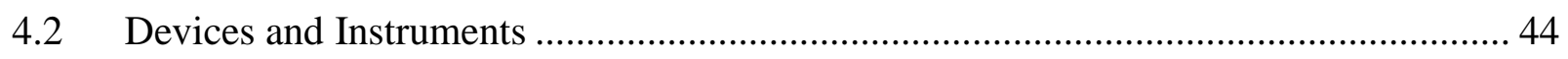

4.3 Configuration of The FBG Pressure Sensor............................................................ 45

5 Experimental Results and Relative Simulation Errors................................................... 48

5.1 Pressure Measurement at Room Temperature ..................................................... 48

5.2 Temperature Measurement at Room Pressure …..................................................... 51

5.3 Simultaneous Pressure and Temperature Measurement............................................ 52

5.4 Time response of Pressure Measurement .......................................................... 54

5.5 Pressure Measurement Versus Simulation .......................................................... 56

6 Conclusion and Future Works .................................................................................. 59

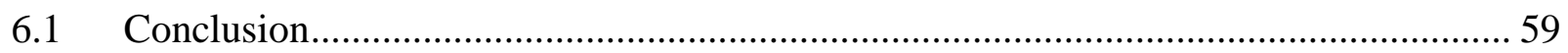

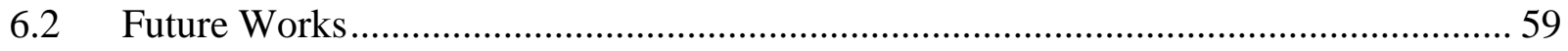

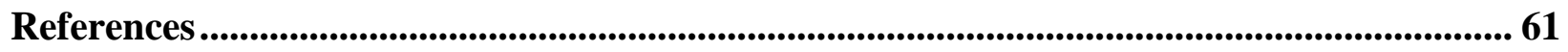




\section{List of Abbreviations}

Abbreviation

Explanation

Unit

$\varepsilon_{R c} \quad$ Radial strain at the center of a diaphragm

$m / m$

$\varepsilon_{T c} \quad$ Tangential strain at the center of a diaphragm

$m / m$

$P \quad$ Pressure

$P a$

$R_{0} \quad$ Radius of diaphragm

m

$v \quad$ Poisson's ratio

$t$

Thickness

$m$

E Young's modulus (modulus of elasticity)

$N / m^{2}$

$q_{p} \quad$ Piezoelectric charge

C

$C_{p} \quad$ Piezoelectric capacitance

F

$C_{c} \quad$ Cable capacitance

F

$R_{f}$ and $R_{g} \quad$ Op-amp resistors values

$\Omega$

$V_{c c} \quad$ Operating voltage

V

T Temperature

K

$R \quad$ Resistance

$\Omega$

$\lambda_{B} \quad$ Central wavelength

$n m(m)$

$n \quad$ Refractive index

$\Lambda \quad$ Grating period or pitch

$n m(m)$

L Length

$m$

$\alpha \quad$ The coefficientof thermal expansion

$\Delta T \quad$ Temperature change

$K$

$\emptyset_{(x, y)} \quad$ Value of field function in point with $\mathrm{x}$ and $\mathrm{y}$ coordinate 


$\begin{array}{clr}\emptyset_{\mathrm{i}} & \text { Amount of field function in boundary nodes } \\ N_{i_{(x, y)}} & \text { Shape functions or blending functions } & \\ \sigma_{i} & \text { Stress component } & \mathrm{N} / \mathrm{m}^{2} \\ C_{1}, C_{2} & \text { Stress-optic coefficients } & \mathrm{Pa}^{-1} \\ v & \text { Poisson's ratio } & -\end{array}$




\section{List of Tables}

Table 1 - summary of some FBG pressure sensor reported ............................................... 16

Table 2 - simulation results summary, wavelength shift due to pressure ................................ 38 


\section{List of Figures}

Figure 1 - (a) and (b) simple mechanical pressure gauge, (c) pressure gauge cross section, (d)

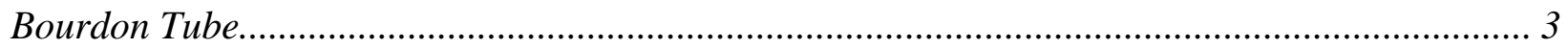

Figure 2 -(a) real strain gauge, (b) foil strain gauge structure, (c) only measuring axial strain 5

Figure 3 - (a) diaphragm pressure transducer, (b) foil strain gauge configuration, $(c)$ radial and

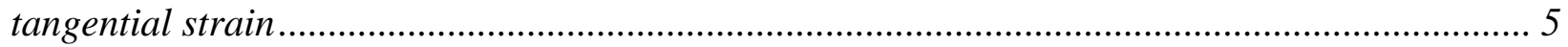

Figure 4-(a) ion displacement, (b) commercial piezoelectric, (c) piezoelectric pressure sensor 7

Figure 5-a sample circuit for piezoelectric sensors and its frequency response offered by Texas

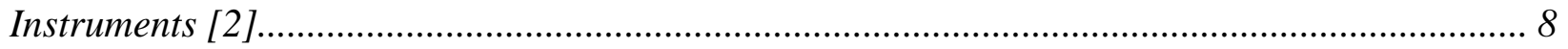

Figure 6 - FBG transmitted signal and reflected component at central wavelength................. 10

Figure 7 - fibers cross section of Chung Wu et al. experiment [6] ....................................... 14

Figure 8 - fibers cross section of Kevin P. Chen et al. experiment [7]................................. 15

Figure 9 - fibers with suspended core and cladding [8] .................................................. 15

Figure 10 - fibers cross section of Sanne Sulejmani et al. experiment [9] ............................... 16

Figure 11 - (a) gridiron pendulum (b) bimetal temperature compensator $(c)$ conceptual pressure transducer with a temperature compensator

Figure 12 - (a) triangle simulation of a circle (b) meshing of a circle with triangular elements (bring the Figure on the same page as the content) ............................................................ 24

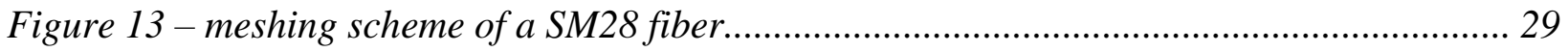

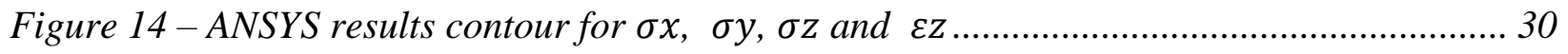

Figure 15-COMSOL results of the electric field of light waves in a microstructured FBG..... 31

Figure 16 - wavelength shift of SM28 FBG under hydrostatic pressure ................................. 32

Figure 17 - (a) side pressure increase the length (b) end pressure reduce the length of fiber.... 33 
Figure 18 - wavelength shifts of SM28 due to (a) side pressure strain (b) side pressure refractive index change, $(c)$ end pressure strain, $(d)$ end pressure refractive index change ........................ 34

Figure 19 - geometry of two FBGs used for simulation...................................................... 36

Figure 20 - total wavelength shifts in small hole FBG .................................................... 37

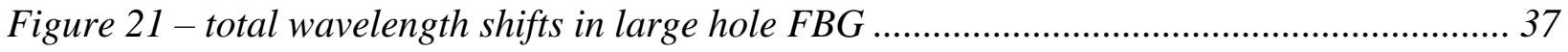

Figure 22 - the effect of core aspect ratio on central wavelength shift ................................... 39

Figure 23 - effect of holes diameter on central wavelength shift .......................................... 40

Figure 24 - distance of holes from the core effect on central wavelength shift ........................ 40

Figure 25 - three different types of FBG pressure sensor setup ........................................... 41

Figure 26 - microstructured fiber cross section used for the experiment ................................. 43

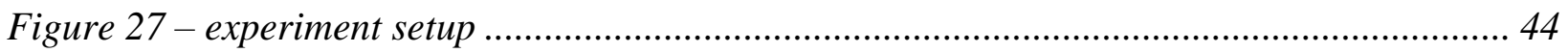

Figure 28 - A reflection spectrum of a typical conventional FBG (red) versus........................ 46

Figure 29 - raw spectra of large hole fiber $\pi$-phase shifted $F B G$......................................... 46

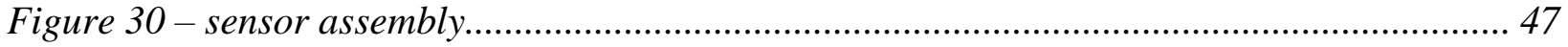

Figure 31 - reflected spectra shift due to rising pressure (a) standard $F B G$ (b) $\pi$-phase shifted

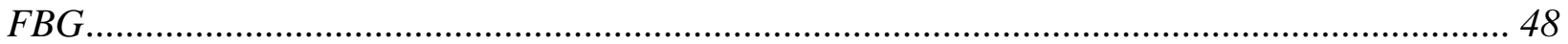

Figure 32 - standard and $\pi$-phase shifted SPF-1550 FBG pressure response ......................... 49

Figure 33 - large hole fiber (a) spectra shift (b) central wavelength shift due to rising pressure 50

Figure 34 - large hole fiber (a) spectra shift (b) central wavelength shift due to rising temperature 51

Figure 35 - pressure measurements in 3 different temperature level (a) fast axis (b) slow axis. 52

Figure 36 - temperature measurements in 4 different pressure level (a) fast axis (b) slow axis. 53 
Figure 37 - time dependency of gas and water pressure measurement in different temperatures

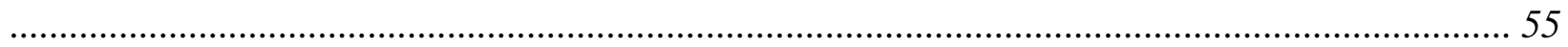

Figure 38 - peaks separation measurement method (a) time dependency $(b)$ pressure measurement 56

Figure 39 - FBG pressure measurement at room temperature simulation and experimental results 57 


\section{Introduction}

Measurement of hydrostatic pressure is a crucial part of many processing lines in the industry from the food and pharmaceutical to Oil and Gas. Pressure measuring devices are used to control the performance of our home heating and cooling systems as well as water supply networks. These devices also play a significant role in the aerospace industry, where the propulsion comes from gas pressure. However, not all of these wide ranging applications require the same accuracy and precision of pressure measurement, since the effect of pressure change is different in each of them. For instance, a small pressure change inside a pressure vessel, which is negligible in an airconditioning system, may cause a substantial change in product yield in a processing line or lead to a devastating explosion in a nuclear reactor. For this reason, a pressure measuring device must be selected based on the characteristic of each application.

Many different types of pressure measuring device have been designed, developed and used based on their application and accuracy requirements. Among these, the low precision mechanical pressure gauge is the simplest and most common type while the electrical one is more complicated and performs with higher precision and accuracy. Different types of electrical pressure sensors are available, such as load cell based sensors, which requires pressure transducer. In addition to the mechanical and electrical, another kind of pressure sensor has been deployed in recent years is fiber optic sensor. Most of the sensors in other measuring applications such as temperature and flow measurement also can be categorized under these three main types of sensors. Each of these technologies has its own advantages and disadvantages. For example, the main advantage of piezoelectric pressure sensors is their high precision and light weight while their main limitation is low sensitivity to static and low frequency loading, and the main drawback of the pressure transducer is their large shape and heavy weight while they are cheaper than many other complicated electrical sensors.

It is not just the technology used in sensor design and manufacturing that determines the precision and accuracy of measurement; environmental effects can also impose a significant impact on the measurement outcomes. Temperature changes, for instance, can degrade the results of pressure or flow measurement, regardless of the sensor type, by causing a change in mechanical and electrical properties of materials which are used both in the sensors and in the corresponding measuring 
instruments. Hence, a reliable measuring system must be able to remove any kind of cross-talk and provide appropriate results that reflect the changes in the main parameter under investigation. This functionality can be achieved by considering some provisions during design and manufacturing of sensing devices and later by applying corrections on the results. One method which is widely used to remove the effect of temperature on other parameters measurement is compensation technique. Temperature compensator is a metallic part designed and attached to the sensor to make it insensitive to temperature changes. In the other approach, an additional temperature sensor is installed in pair with the main sensor and the adjustment is made based on its reading. The former method expands the dimension and weight of the sensor and the later one requires a data processing which means additional time and hardware. There is a third method of environmental effects removal in which both parameters, pressure and temperature for instance, are measured simultaneously. In this technique, which is called discrimination method, the sensor has an ability to respond to the different measuring parameters at the same time and provide separate measerement data based on its mechanical and physical properties. Temperature discriminator sensor has the same dimension as a single sensor and does not need any data processing during and after measurement. A specific type of fiber optic sensors can work as temperature discriminator and measure temperature and pressure at the same time. The following sections contain first an overview of todays sensing technologies and later on, a discussion about the advantages of using fiber optic sensors in pressure measurement.

\subsection{Pressure Measurement}

Sensors can be categorised in several types according to the principles that they use to transfer a physical parameter to the reading data. They can do it either mechanically or electrically or even by a complex combination of mechanical and electrical devices.

\subsubsection{Mechanical Pressure Sensors}

Mechanical sensors use physical phenomena and solid or fluid mechanical rules to measure pressure. They mainly consist of rudimentary parts such as springs, tubes, mechanical counters, levers, etc. 


\subsubsection{Pressure Gauges}

Pressure gauges are the simplest most frequently used pressure measuring instruments that use the elasticity rules to measure pressure. Their pressure sensitive element is known as Bourdon tube named after French engineer Eugène Bourdon who first used this method for pressure measurement. Today we can see these gauges everywhere in industries, in HVAC systems, or even as a gauge for vehicle tires air pressure checking. Figure 1 shows two types of pressure gauge used in the industries such as automotive, as well as a cross section of these devices to illustrate the fundamentals behind this gauges.
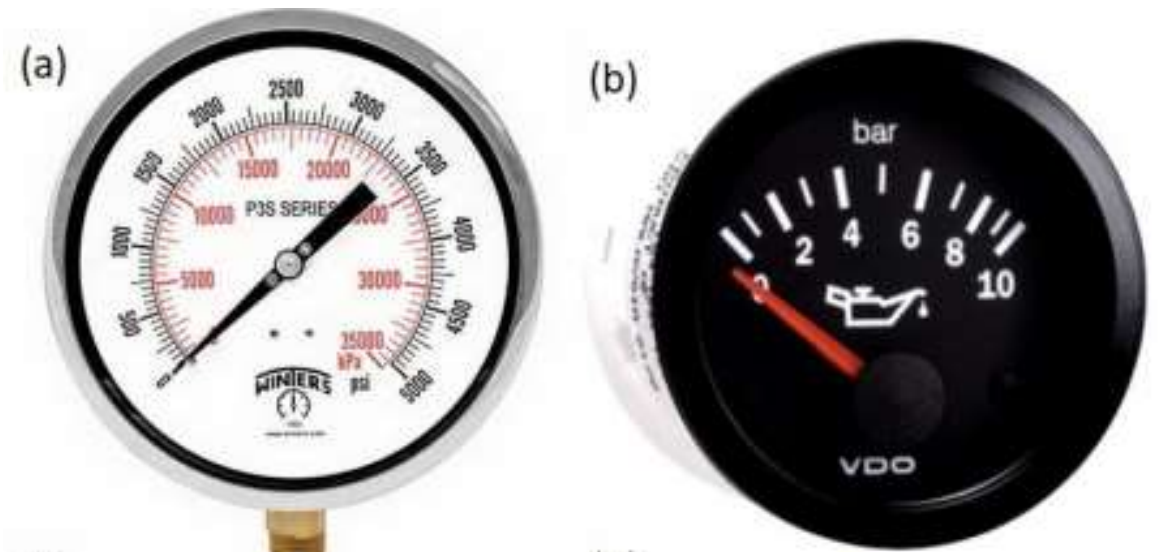

(c)

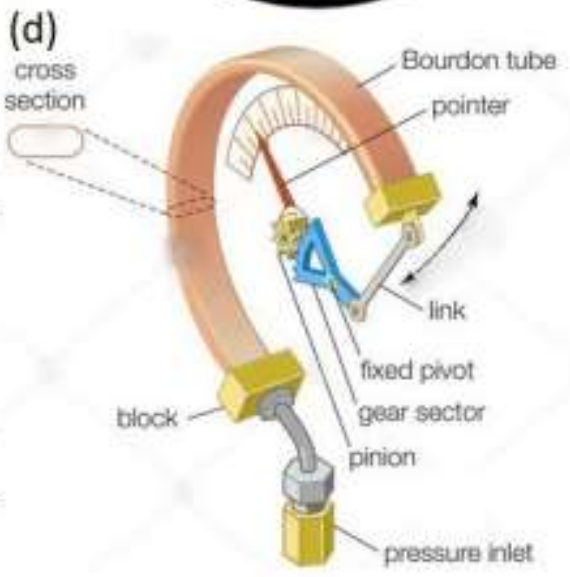

Figure 1 - (a) and (b) simple mechanical pressure gauge, (c) pressure gauge cross section, (d) Bourdon Tube

As it can be seen in Figure 1(d), a Bourdon tube has an elliptical cross section. When the pressure inside this tube increases, its diameters aspect ratio decreases and the structure of the C-shaped tube softens against applied load since the moment of inertia declines along with its strong axis. As a result, the radius of the Bourdon tube curve increases which transfers to the reading pointer by the means of some mechanical parts. Since all parts remain in the elastic domain when the 
pressure value is in the range of gauge manufacturer specification, the measurement has an acceptable linear relationship with applied pressure at a controlled temperature. However, the accuracy of reading is not too high as the values must be read on a scaled surface by eyes. Beside their accuracy and precision limitation, pressure gauges have some other drawbacks prevent them to be used in a wider range of applications. For example, they are sensitive to temperature change, vibration and shock, they are vulnerable to corrosion, fatigue and impact, and they have moving mechanical parts inside them that may affect their durability.

\subsubsection{Electrical Pressure Sensors}

Electrical sensors have dominated our world of technology. They have been made in several types and each of them works based on specific principles of measuring. Furthermore, electrical sensors can be paired with an appropriate data acquisition and data logging system that makes them a preferred choice for monitoring applications such as machine condition monitoring in mechanical equipment. In compare with mechanical sensors, electrical sensors are more accurate and higher precise, more lightweight, smaller, and in many cases more flexible to use. In addition to these advantages, electrical sensors are manufactured in a diverse range of specifications.

\subsubsection{Diaphragm Pressure Transducer (Diaphragm Strain Gauges)}

Before introducing the diaphragm pressure transducer, we must explain how a foil strain gauge works. Foil strain gauges are mainly thin metal resistors with a specified length bent in grids or spiral shape. They are attached to the mechanical parts sustaining stress such that the deformation of the part transfers to them. As the length of the gauge changes due to deformation, its total electrical resistance differs proportional to the elongation or shortening. By measuring the electrical resistance change, the exact length changes of the gauge, i.e. the mechanical strain of the part under investigation, can be calculated. This special mechanism of measurement can cover only axial deformation or strain. However, other types of strain can be transformed to linear deformation by the mean of some mechanical devices called transducer. Figure 2 shows a real strain gauge, its structure, and its dominant measuring direction. 

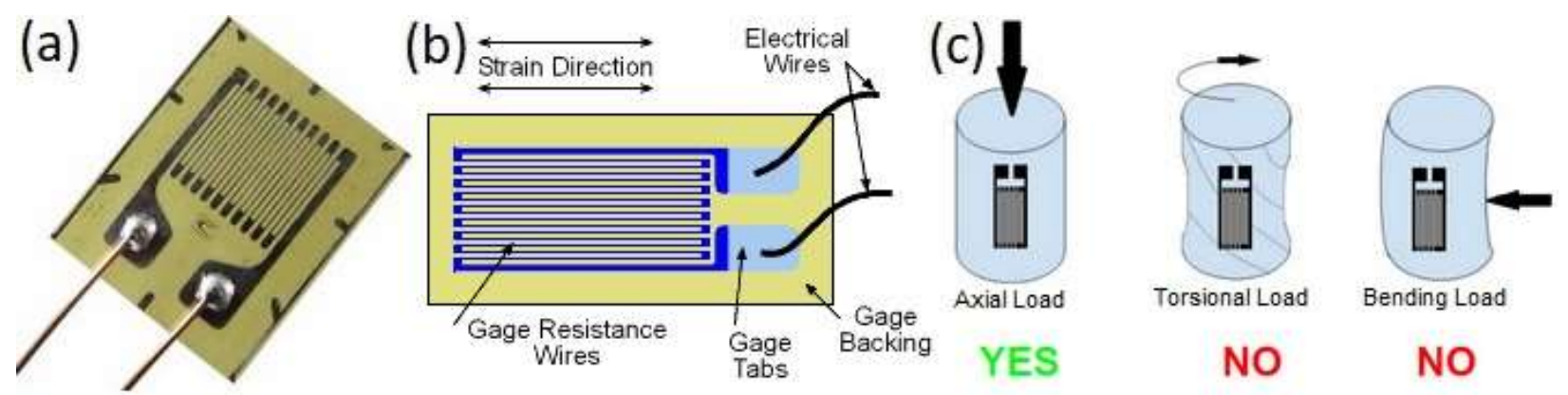

Figure 2 - (a) real strain gauge, (b) foil strain gauge structure, (c) only measuring axial strain

Diaphragm pressure transducer (Figure 3(a)) converts the pressure induced deformation to the strain components (axial deformation) measurable by foil strain gauges.

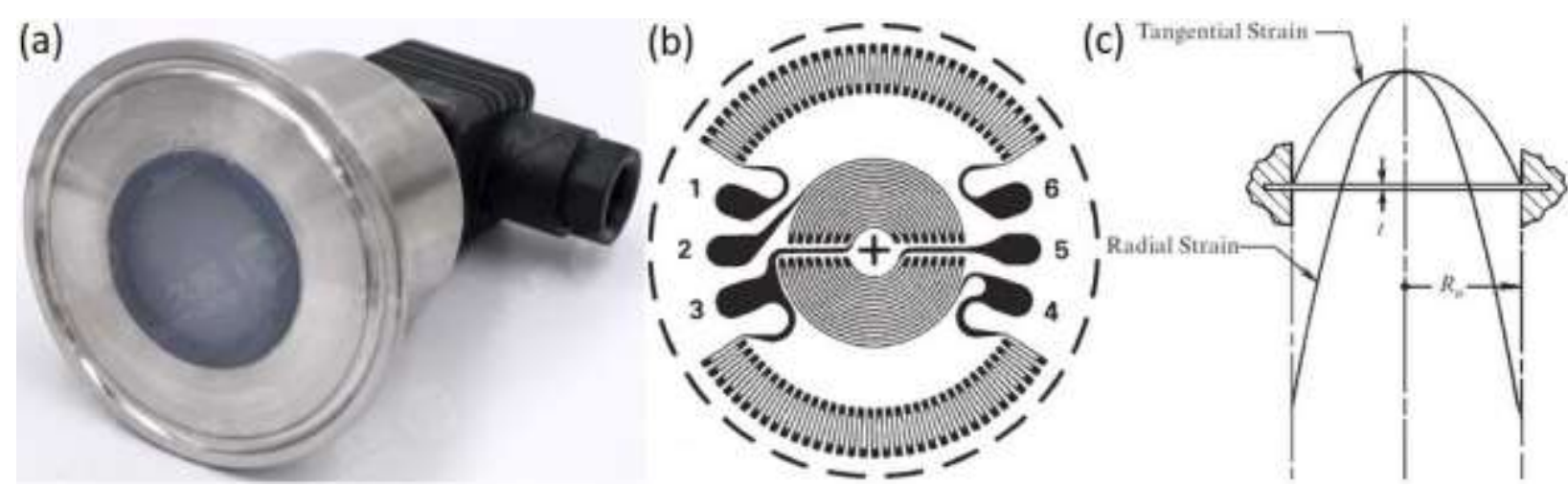

Figure 3 - (a) diaphragm pressure transducer, (b) foil strain gauge configuration, (c) radial and tangential strain

Certainly, the main sensing element of this kind of pressure sensor is a commonly used foil strain gauge, which is connected to the diaphragm exposed to the pressurised fluid. A sample configuration of strain gauges for diaphragm pressure transducer offered by Micro-Measurements is shown in Figure 3(b). Since the outer circumference of the diaphragm is fixed, applied pressure causes a deflection in the diaphragm surface proportional to the pressure. Attached strain gauges measure the strain components of this deformation and the outcomes are used for pressure calculation. Radial and tangential components of the strain in a diaphragm are shown in Figure 3(c) with two separate curves. If the thickness of diaphragm is uniform, the fixing support (clamping) is infinitely rigid, the behaviour of material is perfectly elastic, and if attaching strain gauge to the diaphragm has no effect on its mechanical properties then in small deformations radial and tangential strain of the center point of diaphragm are the same and equal to [1]:

$$
\varepsilon_{R c}=\varepsilon_{T c}=\frac{3 P R_{0}^{2}\left(1-v^{2}\right)}{8 t^{2} E}
$$


where $\varepsilon_{R c}$ is radial strain and $\varepsilon_{T c}$ is tangential strain at the center of diaphragm, $P$ is pressure, $R_{0}$ is diaphragm radius, $v$ is Poisson's ratio, $t$ is diaphragm thickness, and $E$ is Young's modulus or modulus of elasticity. Equation (1) shows that the radial and tangential strain have linear relationship with pressure value and can be used for the purpose of pressure measurement. To do so, the strain gauges that are extended all around the area of diaphragm measure the strain and provide an average value and this value can be considered as $\varepsilon_{R c}$ or $\varepsilon_{T c}$ in equation (1) to calculate applied pressure. In addition to this calculation, equation (1) illustrates that the main principles of this method of measurement come from mechanical relationships. Though, this sensor is not purely electrical and its mechanical mechanism imposes some limitations to its applications quite similar to the mechanical sensor.

Beside the disadvantages come from mechanical parts of diaphragm pressure transducer and the limitations resulting from several assumptions that were made to simplified the calculation of main equation (1), these sensors inherit the drawbacks of ordinary foil strain gauges. Foil strain gauges are made from metals that makes them extremely sensitive to electromagnetic fields. For this reason, outcomes of any strain gauge measurement become totally unreliable in the areas where the electromagnetic radiation exists. Furthermore, the electrical resistance variation due to small strain changes is very low and particularly difficult to detect as a result of the signal to noise ratio in the corresponding electrical circuits. Another limitation of foil strain gauges is originated from a long-time behavior of materials called creep. As time passes and the gauge experiences loading and unloading cycles, a small permanent deformation starts appearing due to creep in gauge material. This deformation adversely affects the accuracy of measurement and can not be easily calculated and compensated. It also must be emphasised that diaphragm pressure transducers tend to change the exact volume of the fluid under investigation to measure the pressure. Without this change, the diaphragm does not deflect and the strain gauge can not read any result. Inappropriately, this volume change causes fluctuation in gas temperature and liquid pressure and consequently reduces the accuracy of measurement.

\subsubsection{Piezoelectric Pressure Sensors}

Piezoelectric crystal has become a popular component in electrical engineering especially in the field of sensors and actuators where electrical and mechanical effects come together. This special 
crystal generates electricity when subjected to mechanical force. That is why it was named "Piezo" which is a Greek term means "to squeeze". Back in 1880, the piezoelectric property of quartz was discovered by the Curie brothers. They saw that the dimensions of a sample quartz crystal change when the electric voltage is applied to it and in the opposite, it produces an electric voltage when its dimensions are changed forcibly [2]. Although crystalline quartz in its natural or processed form is fairly sensitive and reliable piezoelectric material, hundreds of other ceramic and plastic material have acceptable piezoelectric properties for sensing application and piezo actuators production. Some of piezoelectric materials are also sensitive to temperature and radiation.

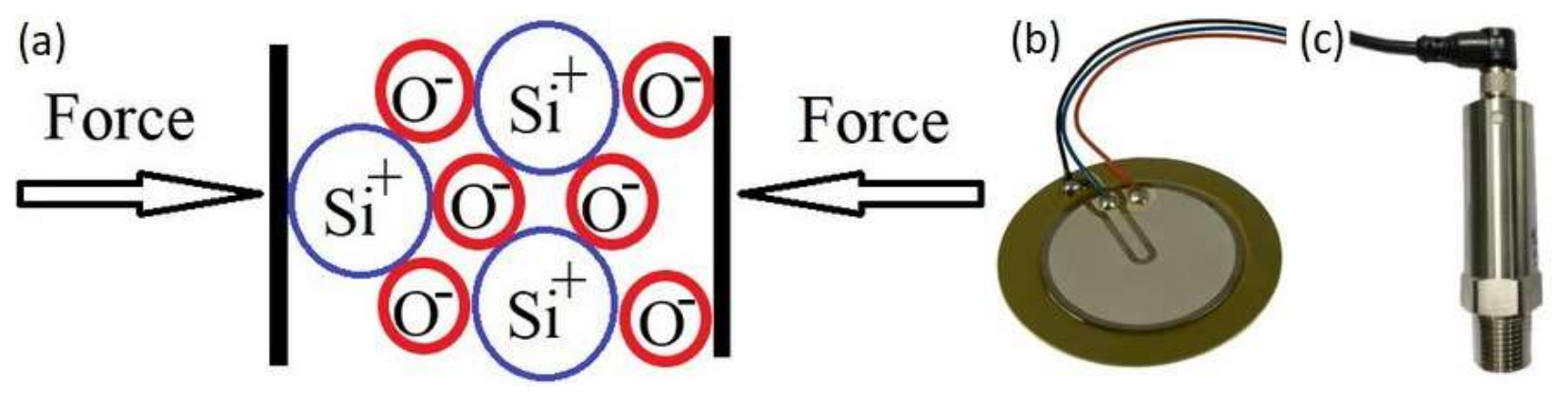

Figure 4 - (a) ion displacement, (b) commercial piezoelectric, (c) piezoelectric pressure sensor

To explain the piezo effect in material, we must look at the molecular level of the crystals. At this level, the structure consists of an ionic bonded crystal. Electrical charges from the ions inside the crystal structure form an electrical dipole. In the neutral condition when the crystal does not experience any load or electric field, its structure is symmetric and the pole shaped by positive ions is exactly similar to that formed by negative ions and they cancel each other. When a mechanical load is applied to the crystal, its dimensions change based on Hooke's law and as a result, its structure becomes asymmetric. This asymmetry creates a net dipole moment and a resulting electric field. The value of this electric charge across the material is proportional to the applied mechanical load [2]. Figure 4(a) shows a quartz crystal consists of silicon and oxygen atoms sustaining axial pressure. As the crystal experiences external stress, electrical charges inside the crystal structure accumulate on two opposite surfaces [3]. Silicon atoms represented by large circles carry positive charges in the lattice and oxygen shown as small circles carry negative charges. Since the electric charge decays rapidly by time due to the internal impedance of both the sensor itself and the instrumentation circuitry, piezoelectric sensors are not suitable for static load measurement. However, they generate an appropriate AC signal in reciprocal loading applications. 
Figure 4(b) shows a commercial piezoelectric and Figure 4(c) depicts a piezoelectric based pressure sensor.

To make a piezoelectric work as a sensor, the electric charge generated by squeezed crystal must be amplified to become a measurable signal. For this purpose, the piezoelectric sensor is considered as a charge source with a shunt capacitor and resistor. The resistance and capacitance of these resistor and capacitor must be selected such that their equivalent impedance become similar to the impedance of the piezoelectric material. The capacitance of piezoelectric sensor can be calculated when its area, width, and dielectric constant are determined. Its resistance also can be estimated based on the dissipation of charge. And finally, the electric charge created by piezoelectric can be evaluated by a parameter called piezoelectric constant.

Figure 5 shows a sample circuit for piezoelectric sensors and its frequency response offered by Texas Instruments [2].

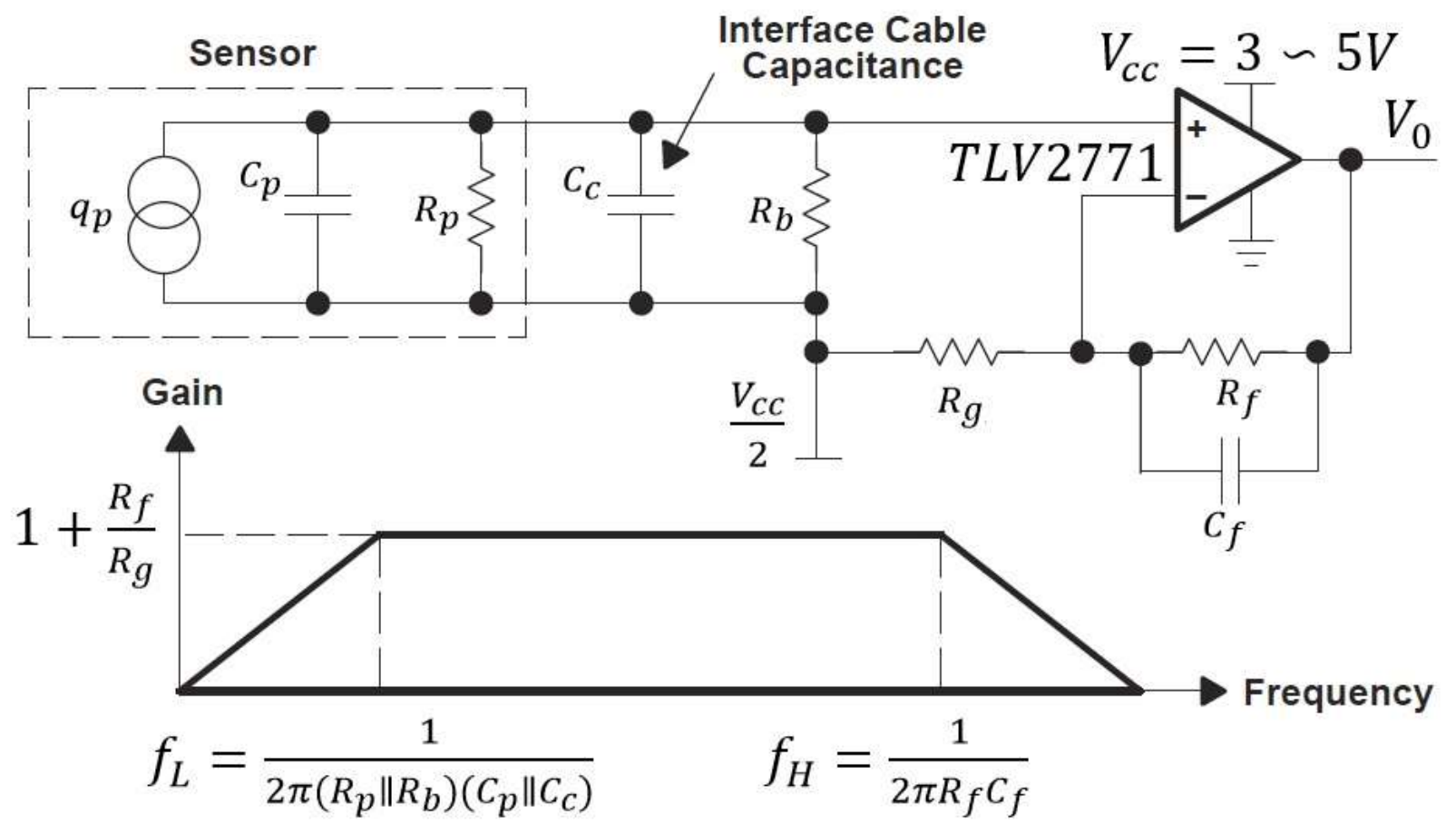

Figure 5 - a sample circuit for piezoelectric sensors and its frequency response offered by Texas Instruments [2]

As it mentioned earlier, in low frequencies the internal impedance of the sensor and circuit can mask the measuring signals. In this sample circuitry, the output voltage is: 


$$
V_{0}=\frac{q_{p}}{C_{p}+C_{c}}\left[1+\frac{R_{f}}{R_{g}}\right]+\frac{V_{c c}}{2}
$$

where $q_{p}$ and $C_{p}$ are piezoelectric charge and capacitance respectively, $C_{c}$ is interface cable capacitance, $R_{f}$ and $R_{g}$ are op-amp resistors and $V_{c c}$ is the operating voltage of circuit.

In addition to their limitation in static load measurement, piezoelectric sensors have other disadvantages such as temperature sensitivity and in some case solubility in water which makes them vulnerable to high humid environments [4]. And as they work based on electric signals, they are susceptible to electromagnetic field quite similar to ordinary strain gauges.

\subsubsection{Fiber Bragg Grating Sensors}

Fiber Bragg Grating (FBG) sensors have become a competitive replacement for ordinary mechanical and electrical sensors in many areas such as stress, temperature, pressure, and flow measurement. Strain measurement with FBGs, for instance, has been widely used in structural health monitoring. Such success cannot be achieved without some advantages like being lightweight, non-conductive, electromagnetic field immune, relatively high sensitive to strain change and the compatibility with wavelength division multiplexing method to measure or monitor several stations on just one line of the fiber optic cable. FBGs do not suffer from creep like foil strain gauge and resistance base thermometer, they can detect static loads unlike piezo sensors since light waves does not decay like electrical signals, they are made of glass and can sustain harsh environmental condition which can effect any other type of sensors, their response is fairly linear to strain and temperature change unlike electrical sensors, they do not have self current, and because of low dimension they respond to temperature changes very quickly. They also can be uses in fluid medium unlike IR temperature detectors. Most of these attributes come from the unique properties of optic fiber.

\subsubsection{Fundamentals of Fiber Grating Sensors}

The main principal of light wave sensing is to detect a particular wavelength shift in the spectra during the change in loading condition [5]. When a spectrum of light passes through an FBG, one particular wavelength is reflected by the grating and all others are transmitted to the other end of fiber. However, in its reflection spectrum, this particular wavelength, which is known as the Bragg 
wavelength of the FBG, can be measured (Figure 6). Equation (3) shows the relationship between the central wavelength and the period of grating:

$$
\lambda_{B}=2 n \Lambda
$$

where $\lambda_{B}$ is the central wavelength, $n$ is the refractive index of FBG core, and $\Lambda$ is the grating period or pitch.

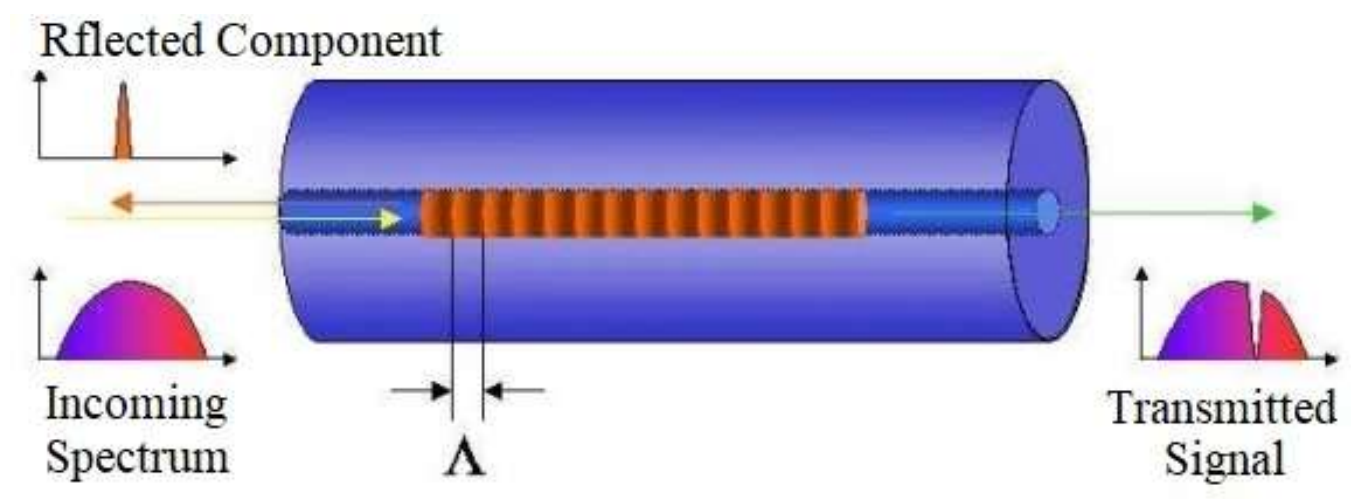

Figure 6-FBG transmitted signal and reflected component at central wavelength

FBGs are attached to mechanical parts for strain measurement. Appling load on a mechanical or structural component causes a change in its dimension according to the mechanical rules such as Hook's law or law of elasticity. This dimensional change transfers to the FBG attached to the element and the period of the FBG change due to strain resulting from deformation. As a consequence of optical parameter (refractive index in particular) variation and grating period change, the Bragg wavelength of the spectrum shifts in a linear relationship with longitudinal strain. To evaluate the shift of the Bragg wavelength due to refractive index and grating period change, by differentiating equation (3) we get:

$$
\Delta \lambda_{B}=2(\Delta n) \Lambda+2 n(\Delta \Lambda)
$$

and dividing both sides by $\lambda_{B}=2 n \Lambda$ we have:

$$
\frac{\Delta \lambda_{B}}{\lambda_{B}}=\frac{2(\Delta n) \Lambda}{2 n \Lambda}+\frac{2 n(\Delta \Lambda)}{2 n \Lambda} \quad \text { or } \quad \Delta \lambda_{B}=\lambda_{B}\left(\frac{\Delta n}{n}+\frac{\Delta \Lambda}{\Lambda}\right)
$$

It is clear from equation (5) that the variation in refractive index and grating pitch must be added together for the wavelength shift calculation in FBG measurement. This equation will be used to 
study the effect of strain, pressure, and temperature on the central wavelength of an FBG in the following sections.

\subsubsection{Pressure Measurement with FBGs}

Many companies offer FBGs pressure sensors based on strain measurement in a tube or diaphragm. As previously stated, FBG sensors can detect extremely low strain changes with high accuracy. If the measuring strain has a predictable relation with applied pressure, the strain value can be interpreted as a pressure level. The device that converts pressure to linear strain is a mechanical apparatus called transducer. Two types of pressure transducer, Bourdon tube and diaphragm transducer were represented in sections 1.1.1.1 and 1.1.2.1 respectively. An FBG pressure sensor consists of an FBG sensor attached to a Bourdon tube filled with pressurized fluid or a diaphragm facing pressurized environment. But not only does this pressure sensor inherit all disadvantages from the mechanical sensor, but also it is tremendously sensitive to ambient temperature change which is an intrinsic attribute of FBG sensors.

Simple FBG sensors can only measure elongation as a linear deformation in the specimen. Nevertheless, when pressure applied to a mechanical component, a complicated combination of other types of strain and stress shaped inside its body. Fortunately, hydrostatic pressure causes axial elongation or shortening in FBGs body that can be easily detected by this type of sensor. In fact, the resultant deformation of the fiber under pressure has a linear relation with the applied pressure which makes it a good parameter for pressure measurement similar to strain measurements with single FBG. This direct pressure sensing helps us to get rid of the drawbacks of using transducers for pressure measurement.

\subsubsection{Temperature Measurement with FBGs}

FBGs can measure temperature precisely since the changes in the terms of equation (5) are proportional to temperature variation. To evaluate the effect of temperature on FBG, we start from simple linear thermal expansion equation from physics and rearrange it to shape the second term in equation (5):

$$
\Delta L=\alpha L \Delta T \quad \text { or } \quad \frac{\Delta L}{L}=\alpha \Delta T
$$


where $L$ is length, $\alpha$ is coefficient of thermal expansion, and $\Delta T$ is temperature change. By considering grating pitch as the specimen length in (6) we will have:

$$
\frac{\Delta \Lambda}{\Lambda}=\alpha \Delta T
$$

which is represents the second term of equation (5). Now, by substituting (7) in (5) and some algebraic simplification we can get:

$$
\Delta \lambda_{B}=\lambda_{B}\left(\frac{\Delta n}{n}+\alpha \Delta T\right) \quad \text { or } \quad \Delta \lambda_{B}=\lambda_{B} \Delta T\left[\frac{\left(\frac{d n}{d T}\right)}{n}+\alpha\right]
$$

In silica fiber, the first term of equation (8) relating to refractive index change by temperature, is considerably higher than the second term which represents thermal expansion in the fiber core. In fact, it dominates the result by contributing to almost $95 \%$ of the central wavelength shift. The

total value of equation (8) brackets is estimated to be $6.67 \times 10^{-6} 1 /{ }^{\circ} \mathrm{C}$ for silica fiber. Having this constant and a wavelength measurement resolution of $1 \mathrm{pm}$, a temperature change of $0.1{ }^{\circ} \mathrm{C}$ can be measured at the central wavelength of $\lambda_{B}=1300 \mathrm{~nm}$ [5].

\subsubsection{Multiplexing Measurement Using WDM Technology}

One of the unique advantages of FBG sensors is their multiplexing capability. They can be multiplexed in a single fiber optic line to transfer data from several FBGs with different central wavelengths. By this technique, several parameters at multiple stations can be measured and transferred through only one fiber optic cable. This conventional method, known as wavelength division multiplexing (WDM), is widely being used in today's telecommunication infrastructure. Theoretically, 1000 different wavelengths can be distributed trough a single mode fibre, ranging from $1200 \mathrm{~nm}$ to $1600 \mathrm{~nm}$ separated $50 \mathrm{GHz}$ from each other. The WDM helps to transfer several independent wavelengths called optical channels through a single fibre optic. In terms of optical networking, wavelength is used to refer to frequency and WDM is similar to frequency division multiplexing (FDM) in radio communication. 


\subsection{A Review of The FBG Pressure Sensing}

As it stated earlier, single FBG sensor can be used to measure pressure without any transducer. In this way, several research and experiments had been reported to evaluate the response of the FBG in different fiber types - also known as microstructured fiber - under direct pressure.

Fibers with non homogeneous cladding known as Polarization Maintain Fibers (PMF) have been developed to provide better control over light wave polarization. However, it has been shown that FBGs written on these types of fiber are more sensitive to pressure than simple single mode fiber FBGs. To explain this effect, we should start from the mechanics of material. When we squeeze the fiber from its side surfaces, the length of fiber increases due to Poisson's effect. On the other side, the pressure effect on the end surface applies compression force that shortens fiber total length. Measuring the difference between these two displacements leads us to a precise pressure measurement. If the fiber has two holes inside its cladding, these holes make the material weaker against applied pressure and according to elasticity law, the fiber will have larger displacement under load. Larger displacement in certain amount of load means larger induced strain and therefore more accurate pressure measurement.

Beside the material properties and structure of fiber optic being used for FBG production, the method of FBG writing also has a considerable effect on pressure measurement. Apparently, more sensitive FBG sensor provides more accurate measurement results regardless of mechanism or type of loading.

As it mentioned earlier, temperature changes effect is another key factor in not only pressure measurement, but also in all kinds of FBG measurement. Therefore, it is important to calibrate the temperature during any experiment or to compensate final outcomes for thermal effects.

Based on these three important factors (structure of the fibre, the method of FBG writing, and temperature control or compensation method) involved in microstructured FBG pressure measurement, several research had been reported to study the reliability and sensitivity of microstructured fiber FBG pressure sensors. Using grapefruit microstructured fibers which has six holes inside its cladding, Chung $\mathrm{Wu}$ et al. compare three different structures of fiber in their experiment [6]. They compared the results of pressure measurement with simple SMF FBG and 
two other PMF FBGs, one had six small and the other had six large holes inside their cladding as shown in Figure 7.
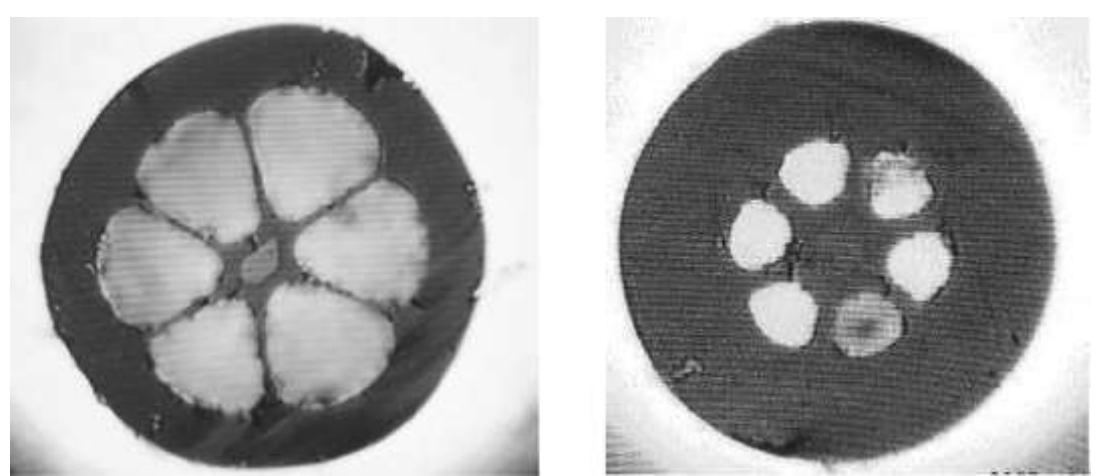

Figure 7 - fibers cross section of Chung Wu et al. experiment [6]

Their measurement was done in room temperature and their FBG writing technology was phase mask. Results of this experiment show -4.23 pm shift in central wavelength per each megapascal pressure change for SMF and $-6.29 \mathrm{pm} / \mathrm{MPa}$ and $-12.86 \mathrm{pm} / \mathrm{MPa}$ for small holes and large hole microstructured fiber respectively. The minus sign indicates that the overall length change of the fiber was negative which means the fiber shrinks under applied pressure. It is clear from their results that increasing the size of holes inside the structure of fiber increases the sensitivity of pressure response.

Another experiment conducted by Kevin P. Chen et al. reported the results of pressure measurement at two different temperatures [7]. The fiber structure consists of two large holes next to the core, and they have used the regenerated grating technique to write their FBG. At room temperature (24 degrees Celsius), they reported a pressure measurement sensitivity of $13.63 \mathrm{pm} / \mathrm{MPa}$. However, this value has been reduced by increasing temperature and reduced to $10.91 \mathrm{pm} / \mathrm{MPa}$ at 800 degrees Celsius. Figure 8 shows the cross section of the fiber used in this study. Different from other experimental results, they reported positive wavelength shift in response to applied pressure. The reason for the positive and negative wavelength shift in pressure measurement will be discussed in section 3.3.2. 


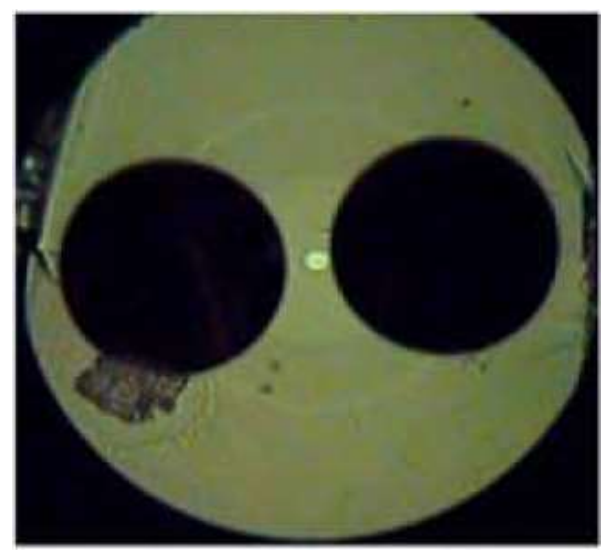

Figure 8 - fibers cross section of Kevin P. Chen et al. experiment [7]

Dr. Tam et al. used a novel fiber type for the purpose of pressure measurement, which consists of a core and cladding which are suspended by a ring inside a larger fiber [8]. As it is shown in Figure 9, this type of fiber provides relatively larger void (air hole) area compared to previous experiments. The measurement was done in controlled temperature condition. The result was $-18.62 \mathrm{pm} / \mathrm{MPa}$ which is obviously caused by shortening of FBG.

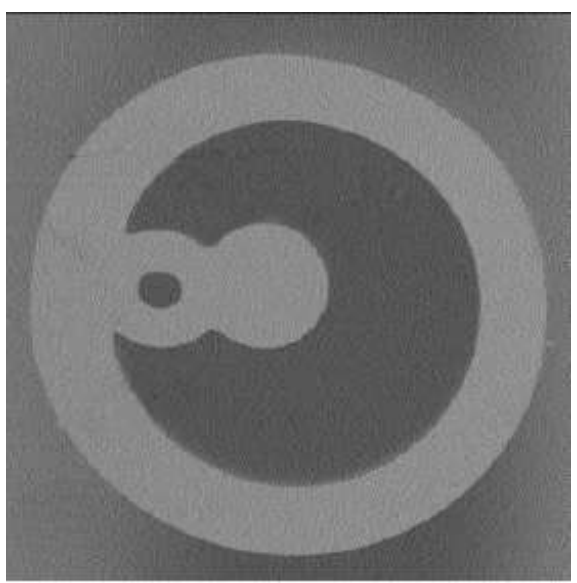

Figure 9 - fibers with suspended core and cladding [8]

FBG produced with different drawing speed is the last experiment we cite here. Sanne Sulejmani et al. used this method to write FBG sensors on small and large hole photonic crystal fibers for their controlled temperature experiment [9]. They reported $-15 \mathrm{pm} / \mathrm{MPa}$ wavelength shift for small hole fiber and $-33 \mathrm{pm} / \mathrm{MPa}$ for large holes fiber respectively. Figure 10 shows the crosssection of the fibers used in their study. 

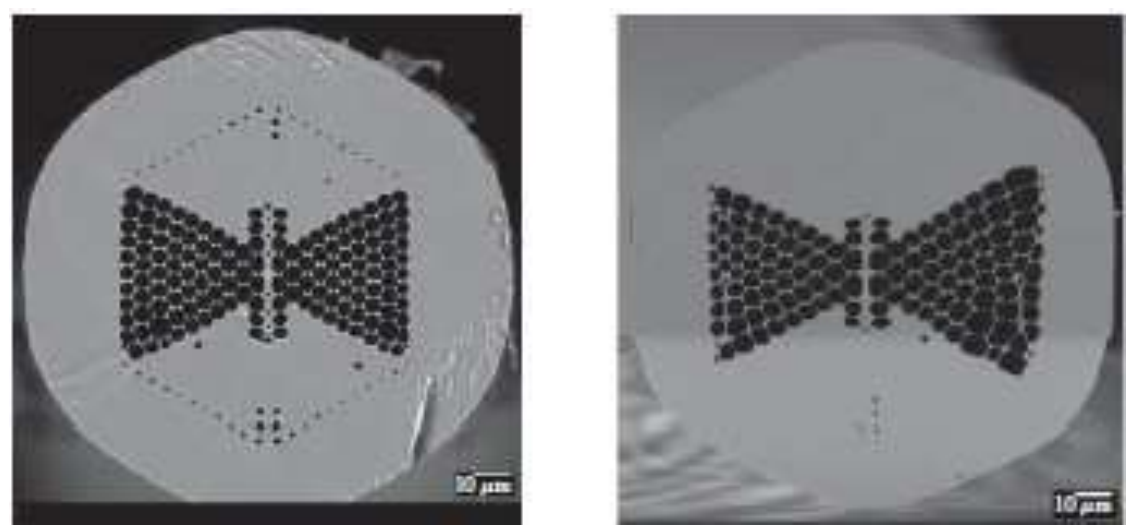

Figure 10 - fibers cross section of Sanne Sulejmani et al. experiment [9]

Table 1 summarizes the setup conditions and the results of these four experiments.

\begin{tabular}{|c|c|c|c|c|c|c|c|c|}
\hline Authors & \multicolumn{3}{|c|}{ Chung Wu et al. } & \multicolumn{2}{|c|}{ Kevin P. Chen et al. } & \multicolumn{2}{|c|}{ Sanne Sulejmani et al. } & Dr. Tam et al. \\
\hline \multirow{3}{*}{\begin{tabular}{|c|c|} 
& Cladding Dia. \\
Fiber Opteic & $(\mu \mathrm{m})$ \\
Specification: & $\begin{array}{c}\text { Hole Dia. } \\
(\mu \mathrm{m})\end{array}$ \\
\end{tabular}} & \multicolumn{3}{|c|}{8} & \multicolumn{2}{|c|}{$9.7 \times 7.5$} & $1.2 \times 3.2$ & $2.6 \times 7.5$ & $5 / 30$ core/cladding \\
\hline & \multicolumn{3}{|c|}{125} & \multicolumn{2}{|c|}{220} & $122 \sim 128$ & $114 \sim 128$ & 125 \\
\hline & 0 & $6 \times 17$ & $6 \times 33.8$ & \multicolumn{2}{|c|}{$2 \times 90$} & \multicolumn{2}{|c|}{-} & Suspended Fiber \\
\hline $\begin{array}{c}\text { Hole } \\
\text { Percentage }\end{array}$ & 0 & 11.1 & 43.9 & \multicolumn{2}{|c|}{33.5} & 35 & 43 & - \\
\hline Sensor Length (mm) & \multicolumn{3}{|c|}{ Not mentioned } & \multicolumn{2}{|c|}{200} & \multicolumn{2}{|c|}{ Not mentioned } & 6 \\
\hline FBG Length (mm) & \multicolumn{3}{|c|}{ Not mentioned } & \multicolumn{2}{|c|}{30} & \multicolumn{2}{|c|}{ Not mentioned } & 1 \\
\hline FBG Writing Technology & \multicolumn{3}{|c|}{ Phase Mask } & \multicolumn{2}{|c|}{ Regenerated Grating } & \multicolumn{2}{|c|}{$\begin{array}{l}\text { Different Drawing } \\
\text { Speed }\end{array}$} & Stack and Draw \\
\hline $\begin{array}{l}\text { Measurement Device } \\
\text { Resolution (pm) }\end{array}$ & \multicolumn{3}{|c|}{ Not mentioned } & \multicolumn{2}{|c|}{20} & \multicolumn{2}{|c|}{1} & 5 \\
\hline Ambient Fluid & \multicolumn{3}{|c|}{ Oil } & \multicolumn{2}{|c|}{ Nitrogen } & \multicolumn{2}{|c|}{ Oil } & Oil \\
\hline Pressure Range (Mpa / bar) & \multicolumn{3}{|c|}{$0.1-24 / 1-240$} & \multicolumn{2}{|c|}{$0.1-16.5 / 1-165$} & \multicolumn{2}{|c|}{$0.1-9 / 1-90$} & $0.1-40 / 1-400$ \\
\hline Temperature & \multicolumn{3}{|c|}{ Room Temp. } & $\begin{array}{c}24{ }^{\circ} \mathrm{C} \\
\text { (Controled) }\end{array}$ & $\begin{array}{c}800{ }^{\circ} \mathrm{C} \\
\text { (Controled) }\end{array}$ & \multicolumn{2}{|c|}{ Controled } & Controled \\
\hline Wavelength Shift (pm/Mpa) & -4.23 & -6.29 & -12.86 & 13.63 & 10.91 & -15 & -33 & -18.92 \\
\hline Regression $R^{2}$ & \multicolumn{3}{|c|}{ Not mentioned } & \multicolumn{2}{|c|}{ Not mentioned } & \multicolumn{2}{|c|}{ Not mentioned } & 0.9997 \\
\hline
\end{tabular}

Table 1 - summary of some FBG pressure sensor reported 


\subsection{Motivation of The Research}

FBG sensing technology can overcome some limitations existed in the mechanical and electrical sensors. It is electromagnetic field immune, light weight and easy to mount, electrically nonconductive, and resistant to harsh environments. Data from FBG sensors can be transfer to the distanced measuring or processing station since the loss in fiber optic is extremely lower than the loss of copper wires used for electrical sensors. Fatigue and time related response drift as a durability issue are negligible in FGB sensing technology.

Despite several advantages, the reported FBG pressure sensors have some drawbacks that prevented them from being widely used in certain applications. They are very sensitive to the ambient temperature. A small temperature fluctuation can deteriorate the pressure measurement results. They are also not adequately sensitive to pressure without using transducers. But as stated previously, using transducers for pressure measurement may introduce new problems to the measuring system since they mostly consist of mechanical components.

To make FBG sensors an acceptable alternative to commercial pressure sensors, I need to address the temperature cross-talk issue and inadequate pressure responsivity issue in this work. Using FBG written in microstructured fiber and measuring two polarization peaks, the temperature can be measured and its cross-talk to the pressure reading can be removed without the need of any mechanical transducer. By the use of $\pi$-phase shifted FBG, whose bandwidth is about 30 to 50 time narrower than that of conventional FBG, the sensitivity of FBG can be greatly improved. In brief, a $\pi$-phase shifted FBG written on a microstructured fibre can measure temperature and pressure simultaneously with acceptable precision and accuracy.

Since FBGs are very thin (less than $200 \mu \mathrm{m}$ in diameter) it does not take much time for the heat to transfer to their core. For this reason, the response time in an FBG temperature measurement should be relatively low. However, there is no study taken yet to evaluate time response of FBG pressure sensors caused by gas law. I examine the effect of time on pressure measurement with a microstructured $\pi$-phase shifted FBG in this work. 


\section{Simultaneous Multi-Parameter Measurement}

Many physical parameters are interactive with each other. In many situations, we need to keep track of multiple parameters to study or to control physical phenomenon. This can be the case in many industrial practices or even biomedical applications. Measuring several parameters at same time without cross-talk, requires appropriate equipment and approved methods.

\subsection{Multi-Parameter Measurement Applications}

Temperature, as an environmental parameter, can undesirably affect the results of mechanical load measurement in two ways. First, the magnitude of mechanical load can be changed at different temperatures. For instance, many materials expand due to rising temperature and this expansion as a length or volume change can increase (or decrease in opposite direction) the amount of mechanical load. Therefore, in industries dealing with fluid in their process line, pressure measurement can be subjected to undesired variation due to temperature fluctuation. The second thermal effect on load measurement is the response of the sensors themselves to the ambient temperature. Many electronics and even mechanical device in sensing procedure are thermal sensitive which means that their response change by temperature. For instance, the electrical resistance of foil strain gauges and the spring constant of mechanical gauges change by temperature.

Although the environmental condition effects such as temperature must be removed from any accurate measurement, in many applications we need to measure the exact value of these environmental parameters for other reasons. As an example, operators in the Oil and Gas industry want to know the pressure and temperature in their pressure vessels or flow rate and temperature in their gas pipes at the same time.

\subsection{Multi-Parameter Measurement Methods}

It is common to use two different sensors for the measurement of two different parameters such as pressure and temperature. This method is highly reliable and straight forward. However, it duplicates the number of sensors and relating instrument which can significantly increase the expenses of installation and maintenance. There are other methods that can measure both 
parameters at the same time or remove the effect of one parameter on the other without doubling the sensors. Compensation method and discrimination technique are two of the most popular choices in the industry and among the researchers.

\subsubsection{Compensation Method}

Compensation method is mainly used to remove the effect of temperature on another measurement. The temperature compensation technique uses the difference between the thermal expansion coefficient of two different materials to eliminate the effect of temperature on sensors. This method is invented by John Harrison in 1726 when he used it to remove thermal effects on his clocks pendulums. He was aware that the length of the pendulum would change due to thermal expansion when the temperature changes. So he used two different metals, steel and brass, which had two different thermal expansion coefficient in his Gridiron Pendulum such that they expanded in opposite directions to cancel out the total elongation. Harrison's invention of gridiron pendulum is considered to be one of the first automatic control systems in the world. A similar setup can remove the thermal effects from sensors using for other parameters measurement. Figure 11(a) shows the gridiron pendulum alongside with Figure 11(b), a bimetal compensator for linear sensors such as FBGs and Figure 11(c), a conceptual drum shaped pressure transducer combined with temperature compensator.

Even though temperature compensation is a simple solution for temperature insensitive measurement in many fields, it has some disadvantages similar to mechanical sensors. This method not only increases the expenses of manufacturing and expand the dimensions of sensors but also compromises the electromagnetic field immunity attribute of optic fiber based sensors since the materials used for the compensator are mainly metals. Metal compartment diminishes the reliability of the sensor in electromagnetic field and reduces its durability in harsh environments.

\subsubsection{Discrimination Method}

An emerging multi-parameter measurement technique called discrimination method has been used by many researchers to overcome the limitation of compensation or correction methods. In this technique, two different mechanical or physical responses of a sensor are considered as measurand for two different parameters. In temperature discriminator, for instance, each sensor has the 
capability of measuring temperature alongside with other parameters simultaneously without the need of any additional parts or any other sensor. This method can be used to measure load and temperature at the same time with a microstructured FBG.

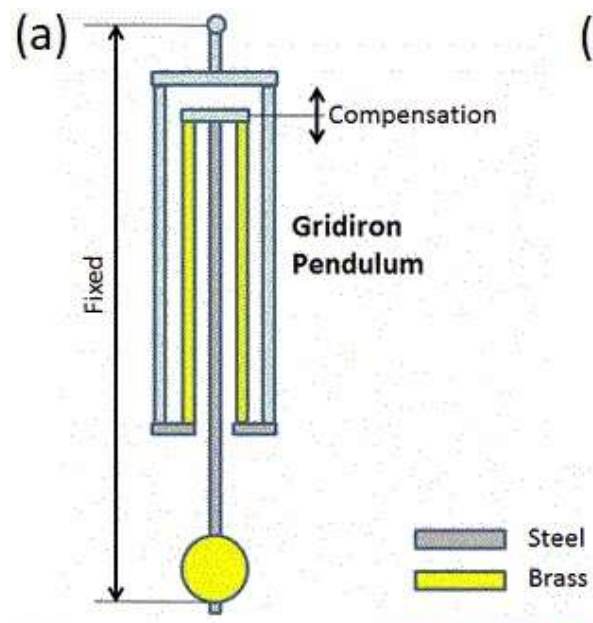

(b)
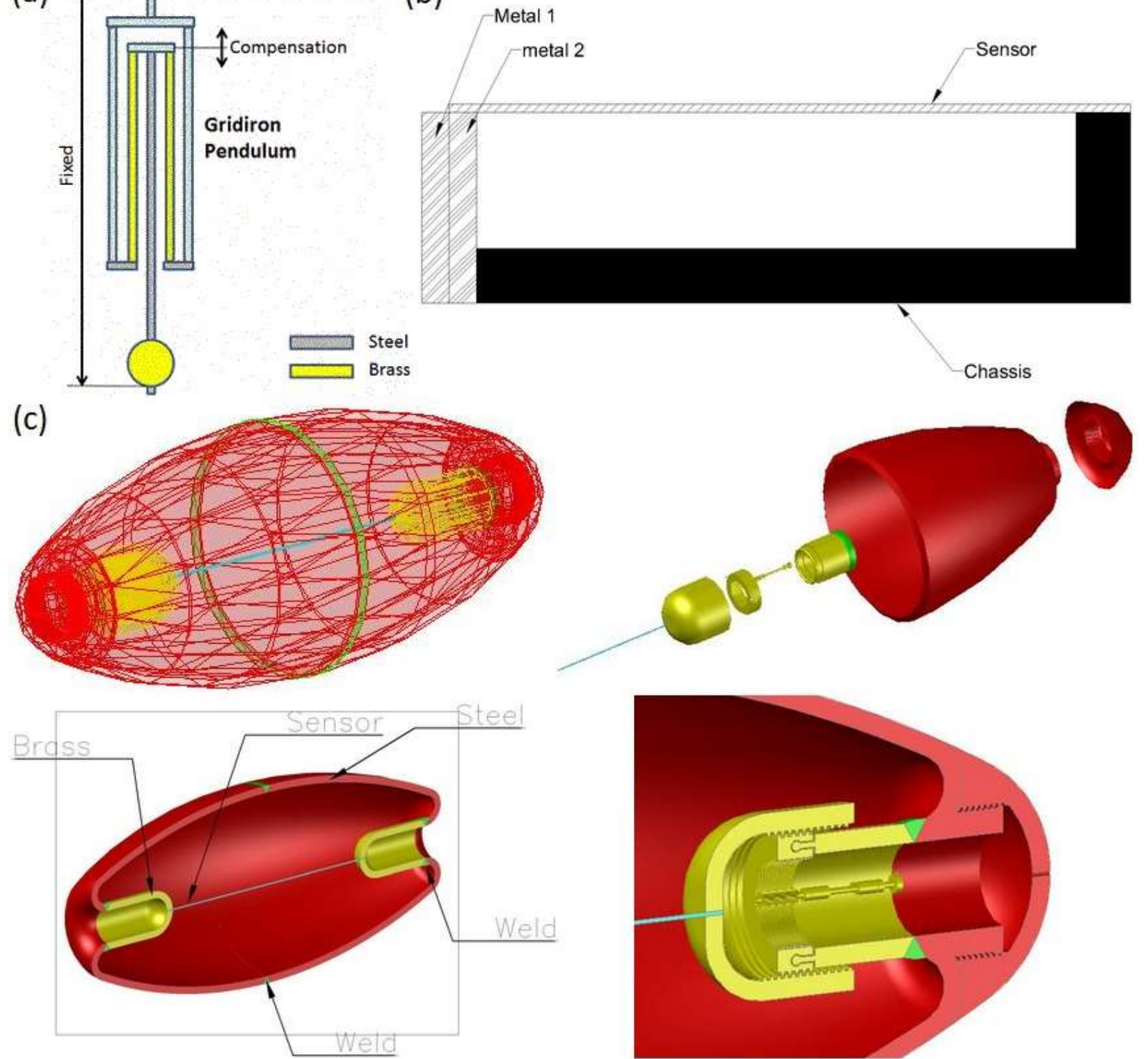

Figure 11 - (a) gridiron pendulum (b) bimetal temperature compensator (c) conceptual pressure transducer with a temperature compensator

In a microstructured fiber, mechanical stresses change differently along two principal axes due to temperature and pressure change (see section 3.2). To measure and calculate temperature and pressure change simultaneously by an FBG, we can combine equation (5) with equation (8): 


$$
\left(\Delta \lambda_{B}\right)=\lambda_{B}\left(\frac{\Delta n}{n}+\frac{\Delta \Lambda}{\Lambda}\right)+\lambda_{B}\left[\alpha+\frac{\left(\frac{(d n)}{d T}\right)}{n}\right] \Delta T
$$

At this time let's consider the term $\lambda_{B}\left(\frac{\Delta n}{n}+\frac{\Delta \Lambda}{\Lambda}\right)$ as wavelength response to pressure change and rewrite equation (9) for two principal axes:

$$
\begin{aligned}
& \left(\Delta \lambda_{B}\right)_{x}=\left(\frac{\left(\Delta \lambda_{B}\right)_{x}}{\Delta P}\right) \Delta P+\lambda_{B}\left[\alpha+\frac{\left(\frac{(d n)_{x}}{d T}\right)}{n_{x}}\right] \Delta T \\
& \left(\Delta \lambda_{B}\right)_{y}=\left(\frac{\left(\Delta \lambda_{B}\right)_{y}}{\Delta P}\right) \Delta P+\lambda_{B}\left[\alpha+\frac{\left(\frac{(d n)_{y}}{d T}\right)}{n_{y}}\right] \Delta T
\end{aligned}
$$

If the temperature change does not affect the response of FBG to stress and strain, which is known as second order effect, the first term of equations (10) can be considered as pressure related wavelength shift while the second term represents the shift due to temperature change. In this condition, these equations can be reduced to:

$$
\begin{aligned}
& \left(\Delta \lambda_{B}\right)_{x}=\frac{\partial\left(\Delta \lambda_{B}\right)_{x}}{\partial P} \Delta P+\frac{\partial\left(\Delta \lambda_{B}\right)_{x}}{\partial T} \Delta T \quad \text { and } \\
& \left(\Delta \lambda_{B}\right)_{y}=\frac{\partial\left(\Delta \lambda_{B}\right)_{y}}{\partial P} \Delta P+\frac{\partial\left(\Delta \lambda_{B}\right)_{y}}{\partial T} \Delta T
\end{aligned}
$$

or in matrix form:

$$
\left[\begin{array}{l}
\left(\Delta \lambda_{B}\right)_{x} \\
\left(\Delta \lambda_{B}\right)_{y}
\end{array}\right]=\left[\begin{array}{ll}
\frac{\partial\left(\Delta \lambda_{B}\right)_{x}}{\partial P} & \frac{\partial\left(\Delta \lambda_{B}\right)_{x}}{\partial T} \\
\frac{\partial\left(\Delta \lambda_{B}\right)_{y}}{\partial P} & \frac{\partial\left(\Delta \lambda_{B}\right)_{y}}{\partial T}
\end{array}\right]\left[\begin{array}{l}
\Delta P \\
\Delta T
\end{array}\right]
$$

and in a more general case when the second order effects are taken into consideration:

$$
\left[\begin{array}{l}
\left(\Delta \lambda_{B}\right)_{x} \\
\left(\Delta \lambda_{B}\right)_{y}
\end{array}\right]=\left[\begin{array}{lll}
\frac{\partial\left(\Delta \lambda_{B}\right)_{x}}{\partial P} & \frac{\partial\left(\Delta \lambda_{B}\right)_{x}}{\partial T} & \frac{\partial^{2}\left(\Delta \lambda_{B}\right)_{x}}{\partial P \partial T} \\
\frac{\partial\left(\Delta \lambda_{B}\right)_{y}}{\partial P} & \frac{\partial\left(\Delta \lambda_{B}\right)_{y}}{\partial T} & \frac{\partial^{2}\left(\Delta \lambda_{B}\right)_{y}}{\partial P \partial T}
\end{array}\right]\left[\begin{array}{c}
\Delta P \\
\Delta T \\
\Delta P \Delta T
\end{array}\right]
$$

using matrices algebra, we can calculate temperature and pressure changes from two polarization modes wavelength shift as: 


$$
\left[\begin{array}{l}
\Delta P \\
\Delta T
\end{array}\right]=[K]^{-1}\left[\begin{array}{l}
\left(\Delta \lambda_{B}\right)_{x} \\
\left(\Delta \lambda_{B}\right)_{y}
\end{array}\right]
$$

where the $[\mathrm{K}]$ is the sensitivity matrix in (12).

We can measure both wavelength shifts along two principal axes of FBG and use the results to solve equation (14) for pressure and temperature changes. 


\section{Finite Element Analysis Simulation of FBG}

Finite Element Analysis is a numerical solution for boundary value problem. Boundary value problem itself, is a differential equation with known values in their boundaries. By the definition, differential equations can have different variables in different fields of study which is called Field Variables. As a result, although FEA has a tremendous popularity among mechanical engineers, it can be used in other physics rather than mechanical stress and strain calculation such as heat transfer or electromagnetic wave propagation. Like any other numerical approaches, FEA results encounter with computational error which must be taken into account in any design and evaluation.

\subsection{Finite Element Method Principles}

The lines, plates or volumes which are considered for finite element analysis in single, two or three dimensional space are called the domain. In case of stress calculation with stress as a field variable, the differential equation represents mechanical properties of domain material and the results of the solution of this equation are the stresses. The exact solution output contains all stress components in every single point of the domain. However, it is almost impossible to calculate infinite numbers of point in a real problem using a mathematical or even computational approach. As a solution to this, FEM suggests to discretize the domain to a finite number of adequately small subdomains which are called elements. The points used for discretization are known as nodes and the line, area or volume between them are called elements. The values of field function are calculated explicitly on these nodes instead of all points inside the domain. This procedure is also known as simulation. For instance, we can simulate a circular disk with a triangle as shown in Figure 12(a) and solve the differential equation for three vertices of the triangle. These three nodes (vertices) are placed on the boundary line of the disk and can be affected by the outside-the-domain circumstances. For this reason, they are called exterior nodes or boundary nodes. A boundary condition problem should have known-values for field variable at its boundaries. In the simulation of a disk with a triangle, the values of field variable in all three nodes which are apparently exterior nodes must be known. Although the values are known at vertices of the triangle, most of the time an estimation of field variable for the points inside the element is required. To evaluate this, interpolation methods can be implemented. In the case of three nodes elements in Figure 12(a), the following interpolation formula can be used to calculate field variable in each point of a triangular element: 


$$
\emptyset_{(x, y)}=N_{1_{(x, y)}} \times \emptyset_{1}+N_{2_{(x, y)}} \times \emptyset_{2}+N_{3(x, y)} \times \emptyset_{3}
$$

In which $\emptyset_{(x, y)}$ is the value of field function at the point with $\mathrm{x}$ and y coordinate, $\emptyset_{1}, \emptyset_{2}$ and $\emptyset_{3}$ are the amount of field function in boundary nodes and $N_{1_{(x, y)}}, N_{2_{(x, y)}}$ and $N_{3_{(x, y)}}$ are interpolation functions which are also known as shape functions or blending functions. Shape functions are usually in the polynomial form of independent variables which are satisfy some conditions at the nodes. Although a good estimation can be achieved for any point inside the element by equation (15) and its relating shape functions, there are several points inside the domain but outside the element in which the field variable can not be calculated and evaluated since we simulate a circle with a triangle. To resolve this issue, we must use more nodes on boundary and more element inside the domain by increasing the number of discretization and reducing the size of triangular elements as illustrated in Figure 12(b). This procedure known as meshing. To increase the number of mesh elements, we require several nodes inside the domain which are called interior nodes. To solve an FEM problem, the value of field function must be calculated at all nodes. It should be noted that some field variables are not scalar and require more than one value to become determined.
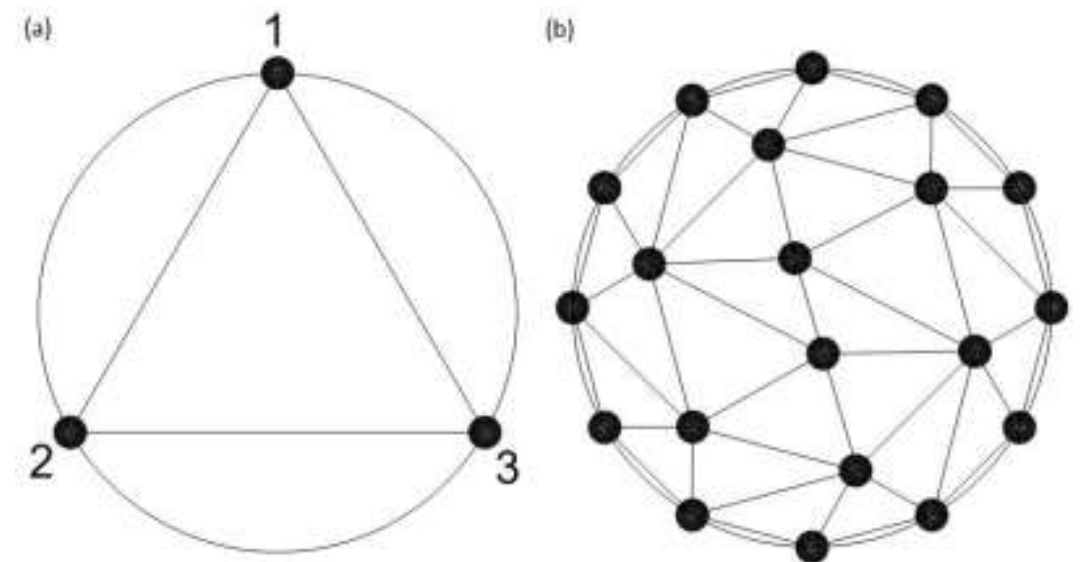

Figure 12 - (a) triangle simulation of a circle (b) meshing of a circle with triangular elements (bring the Figure on the same page as the content)

The total number of values that should be calculated in a finite element analysis is the multiplication of nodes by the number of values that the field function requires for determination. This is known as number of degree of freedom. For instance, in the single-triangle simulation of a circle, if the field variable is temperature which is a scalar of only one value, the number of degree of freedom will be the multiplication of number of nodes which is 3 and number of field variable value which is 1 . However, if we want to calculate the stress which has two components in a two- 
dimensional domain, the number of degree of freedom will be $2 \times 3=6$ [10]. Obviously, the number of degree of freedom increases by the number of mesh elements. In a three dimensional domains when a non-scalar variable like stress and strain, which have three value for each node, is calculated, we are facing a huge number of degree of freedom in real complicated geometries. Solving the differential equation for every single node of the domain using numerical method is a time consuming procedure which in most cases can not be done mathematically. There are several commercial computer applications available to solve the FEM problem in each particular area. ANSYS mechanical and ANSYS AIM are two main products which can simulate and calculate stress and strain in structural and mechanical problems. Ansys incorporated also has several products for other fields such as electromagnetics and fluid mechanics, all of them use FEM as the main approach for calculation of physical parameters across the domains. Other popular FEM software is COMSOL Multiphysics. COMSOL has a great advantage of containing several modules for different areas of physics in a single package which can be combined easily together to study more complicated phenomena. For instance, a mechanical part can be simulated by the solid module of COMSOL and calculated results can be used in another module such as electromagnetic wave at the same application to evaluate the effect of deformation due to mechanical load on the propagation of electromagnetic wave in a specimen. This makes this application very flexible for cross-disciplinary studies. Although these applications make the finite element analysis easy to use, in some cases when very complicated geometries or a high number of degree of freedom is the case, even powerful computers cannot handle the huge amount of data processing required for simulation. In these cases, some simplification based on theoretical or geometrical aspects must be considered to reduce the degrees of freedom. For example, in symmetrical geometries, half of the domain can be simulated for calculation purpose and the outcomes can be used for other half as well.

\subsection{FBGs Mechanical Behavior}

To evaluate the mechanical behaviour of the FBG sensor under pressure, let's start from the main equation (5). To estimate the change of central wavelength with this equation, both terms of $\Delta n / n$ and $\Delta \Lambda / \Lambda$ must be calculated. Changing in reflection index caused by stress inside the core material is called photoelastic effect and can be evaluated by [11]: 


$$
n_{i}-n_{0}=\left(C_{2}-C_{1}\right) \sigma_{i}-C_{2} \sum_{i} \sigma_{i}(i=x, y, z)
$$

in which $i$ represents the principal axes of applied stress, $n_{0}$ is effective refractive index of the core, $n_{i}$ is the refractive index of the core after applying stress in $i$ direction, $\sigma_{i}$ is stress component in $i$ direction, and $C_{1}$ and $C_{2}$ are stress-optic coefficients which are equal to $6.5 \times 10^{-13} \mathrm{~Pa}^{-1}$ and $4.2 \times 10^{-12} \mathrm{~Pa}^{-1}$ respectively for the silica. If the light wave travel along the $\mathrm{Z}$ axis, only $\mathrm{X}$ and $Y$ directional change of $n$ can affect wavelength shift and equation (16) becomes:

$$
\begin{aligned}
& \Delta n_{x}=n_{x}-n_{0}=-C_{1} \sigma_{x}-C_{2}\left(\sigma_{y}+\sigma_{z}\right) \quad \text { and } \\
& \Delta n_{y}=n_{y}-n_{0}=-C_{1} \sigma_{y}-C_{2}\left(\sigma_{x}+\sigma_{z}\right)
\end{aligned}
$$

On the other hand, changing in the Bragg grating period divided by grating pith means lengthchange divided by length which is the mechanical definition of strain. And again if light wave travels along the $\mathrm{Z}$ axis, $\Delta \Lambda / \Lambda$ refers to the $\mathrm{Z}$ axis component of strain. Combining (5) and (17), we have:

$$
\begin{aligned}
& \left(\Delta \lambda_{B}\right)_{x}=\lambda_{B}\left(\frac{-C_{1} \sigma_{x}-C_{2}\left(\sigma_{y}+\sigma_{z}\right)}{n_{0}}+\varepsilon_{z}\right) \quad \text { and } \\
& \left(\Delta \lambda_{B}\right)_{y}=\lambda_{B}\left(\frac{-C_{1} \sigma_{y}-C_{2}\left(\sigma_{x}+\sigma_{z}\right)}{n_{0}}+\varepsilon_{z}\right)
\end{aligned}
$$

Which means when a force is applied on FBG, its central wave length shifts in relation with its strain component in the direction of travelling light wave as well as its stress components in all three Cartesian directions. Equations (18) show that if stresses along fiber cross section main axes are different, wavelength shifts differently in $\mathrm{X}$ and $\mathrm{Y}$ polarization of light beam and causes birefringence in the fiber optic.

If the fiber structure, including cross section and material properties, is symmetric about $\mathrm{X}$ and $\mathrm{Y}$ axes and the load is applied similarly in both directions, neglecting the mechanical effect of cladding on stress distribution, the $\sigma_{x}$ and $\sigma_{y}$ become equal and equations (18) reduce to:

$$
\left(\Delta \lambda_{B}\right)_{x}=\left(\Delta \lambda_{B}\right)_{y}=\lambda_{B}\left(\frac{-\left(C_{1}+C_{2}\right) \sigma_{x}-C_{2} \sigma_{Z}}{n_{0}}+\varepsilon_{z}\right)
$$


from Hooke's law:

$$
\sigma_{z}=E \varepsilon_{z} \quad \text { and } \quad \sigma_{x}=E \varepsilon_{x}
$$

in which ' $E$ ' is Young's modulus.

Combining (19) and (20):

$$
\left(\Delta \lambda_{B}\right)_{x}=\left(\Delta \lambda_{B}\right)_{y}=\lambda_{B}\left(\frac{-\left(C_{1}+C_{2}\right) \sigma_{x}-C_{2} E \varepsilon_{z}}{n_{0}}+\varepsilon_{z}\right)
$$

In many cases, the FBG is used for measuring one dimensional (longitudinal) strain. When the FBG experiences longitudinal stress, according to Poisson's effect the strain in X or Y direction is related to strain in the $\mathrm{Z}$ direction by:

$$
\varepsilon_{x}=v \varepsilon_{z}
$$

where $v$ is known as Poisson's ratio. Putting (22) in (20) results in:

$$
\sigma_{x}=E \times \varepsilon_{x}=E \times v \times \varepsilon_{z}
$$

now by putting (23) in (21):

$$
\begin{aligned}
& \Delta \lambda_{B}=\lambda_{B}\left(\frac{-\left(C_{1}+C_{2}\right) E \times v \times \varepsilon_{z}-C_{2} E \varepsilon_{z}}{n_{0}}+\varepsilon_{z}\right) \quad \text { or } \\
& \Delta \lambda_{B}=\lambda_{B}\left(\frac{-\left(C_{1}+C_{2}\right) \times v-C_{2}}{n_{0}} \times E+1\right) \times \varepsilon_{z}
\end{aligned}
$$

Equation (24) represents the relation between wavelength shift and the longitudinal strain of FBG in symmetrical cross section fiber under pure one dimensional tension or compression. For ordinary single mode fibers, Young's modulus of the core is $69.5 \mathrm{GPa}$, Poisson's ratio is 0.165 and reflective index is 1.45 at $\lambda=1550 \mathrm{~nm}$. Substituting these values and $C_{1}$ and $C_{2}$ in (24) we have:

$$
\begin{array}{cc}
\Delta \lambda_{B}=\lambda_{B} \times 0.76 \times \varepsilon_{Z} \quad \text { or } \\
\frac{1}{\lambda_{B}} \times \frac{\Delta \lambda_{B}}{\Delta \varepsilon}=0.76 \times 10^{-6} \mu \varepsilon^{-1}
\end{array}
$$


which is exactly the same value mentioned in many literatures and approved by many experiments. For $\lambda=1550 \mathrm{~nm}$, the value of equation (25) becomes $\Delta \lambda_{B}=1.2 \mathrm{~nm} / 1000 \mu \varepsilon(\mathrm{pm} / \mu \varepsilon)$ This simple expression can be used for theoretical evaluation of wavelength shift due to one dimensional strain. Please note that the stiffness of the cladding is not considered for the sake of simplification. As it mentioned earlier, these calculations are valid when the cross section of fiber used for FBG is symmetric about its main axes and the load is applied in the direction of fiber as a tension or compression load. If these two requirements are not met, for instance if the cross section of fiber contain some asymmetric parts such as elliptical core or if the FBG is put under more complicated load sequences like hydrostatic pressure, then all parameters in equations (18) should be calculated separately. Calculation of stress and strain requires an appropriate method which can return accurate results in reasonable time. I use FEM for this purpose in the following section to analyze the effect of loads on FBGs.

\subsection{ANSYS and COMSOL Simulation Results}

As it mentioned in the previous section, implementation of equations (18) is impossible without using an FEM software in many cases. In this section, ANSYS AIM software is used to calculate required components of stress and strain inside the structure of FBG to evaluate the effect of load (displacement) on its optical behaviour. Let's get started with a simple FBG written on SMF-28 fiber sustaining a direct tension. The outcome of the simulation of this FBG must be comparable to the mathematical result represented in equation (25). The mechanical and optical properties of the material used for this simulation are as following: for silica cladding, Young's modulus ( $\left.E_{C l a d}\right)$ is 73.1 GPa, Poisson's ratio $\left(v_{\text {Clad }}\right)$ is 0.17 and for germanium doped core, Young's modulus $\left(E_{\text {Core }}\right)$ is $69.5 \mathrm{GPa}$, Poisson's ratio $\left(v_{\text {Core }}\right)$ is 0.165 and reflective index $\left(n_{\text {Core }}\right)$ is 1.46 at $\lambda=1550 \mathrm{~nm}$.

The first step in finite element simulation after geometry and material properties definition is meshing. As discussed earlier, simulation with finer mesh returns more accurate results while requires more physical memory and takes longer time to complete. However, using the symmetrical properties of the model, we can reduce the overall dimensions and decrease the number of mesh elements. Figure 13 shows the meshing scheme of an SMF-28 fiber. It can be seen that the number of mesh elements for the core is considered relatively higher than cladding 
relating to their dimension. This provides more accurate results inside the core which is the main area of interest in stress and strain calculation.

Since the tension inside a one-dimensional bar remains the same along its length, we can freely scale fiber and FBG length to achieve a reasonable number of elements. The fiber in this simulation has the total length of $10 \mathrm{~mm}$ with $3 \mathrm{~mm}$ FBG in the middle of it. There is $1000 \mu \varepsilon(1000 \mu \varepsilon \times$ $10 \mathrm{~mm}=0.01 \mathrm{~mm}$ of displacement) applied on the one end of fiber while other end considered as a fixed support. The simulation results of strain and stress components $\sigma_{x}, \sigma_{y}, \sigma_{z}$ and $\varepsilon_{z}$ in the core area are shown in Figure 14. The small negligible difference between $\sigma_{x}$ and $\sigma_{y}$ in FEM result comes from meshing and numerical calculation procedure error.

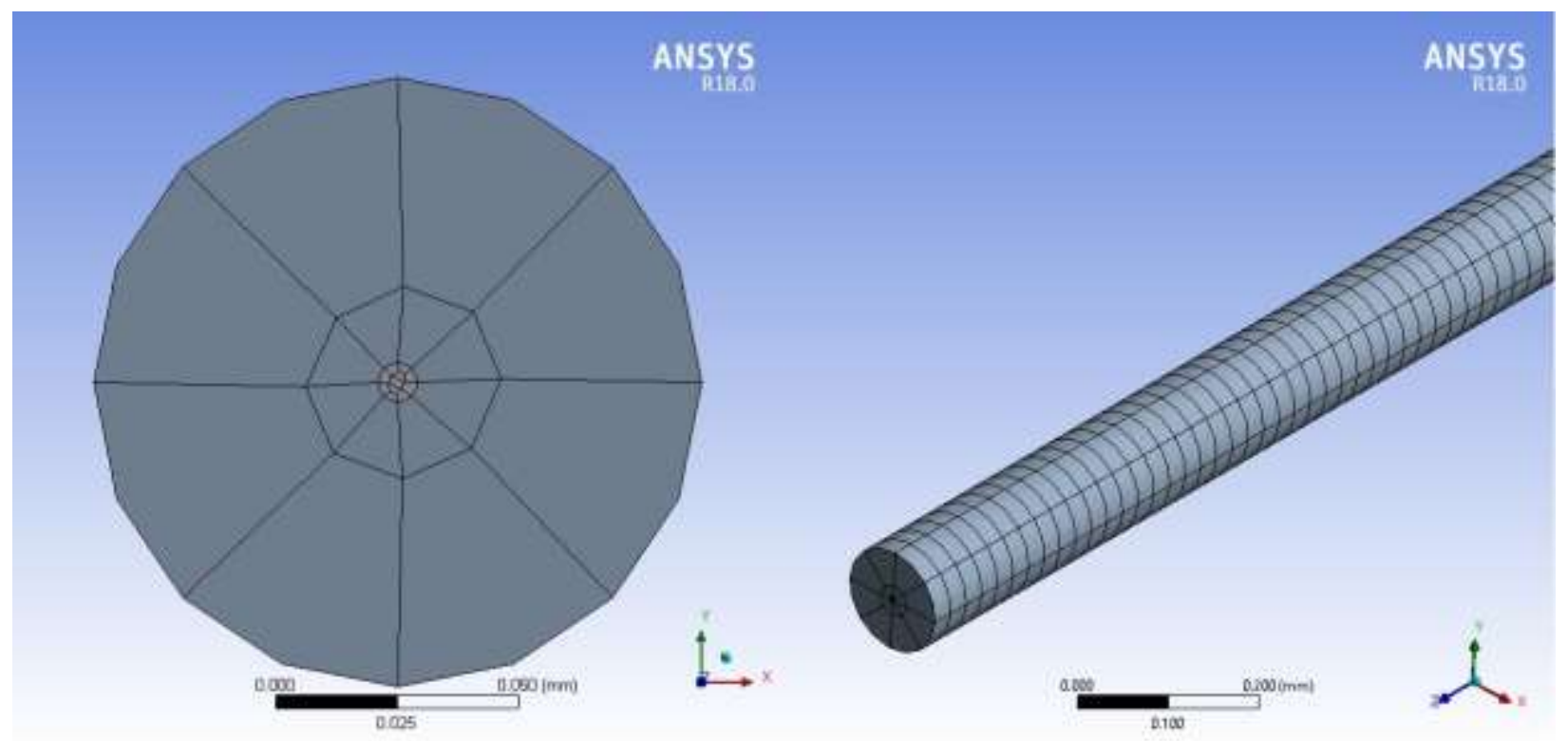

Figure 13 - meshing scheme of a SM28 fiber

Now we can use the FEM results to calculate the wavelength shift by the equation (19). Putting the results shown in Figure 14 into (19), we have:

$$
\begin{gathered}
\left(\Delta \lambda_{B}\right)_{x}= \\
1550 \times\left(\frac{-6.5 \times 10^{-13} \times-0.18 \times 10^{6}-4.2 \times 10^{-12} \times\left(-0.18 \times 10^{6}+69.45 \times 10^{6}\right)}{1.46}+\varepsilon_{z}\right) \\
=1550 \times\left(-1.9919 \times 10^{-4}+10 \times 10^{-4}\right)=1.241 \mathrm{~nm}
\end{gathered}
$$


for $1000 \mu \varepsilon$, which is in agree with the value of $\Delta \lambda_{B}=1.2 \mathrm{~nm} / 1000 \mu \varepsilon$ we obtained in the previous section from theoretical calculation.
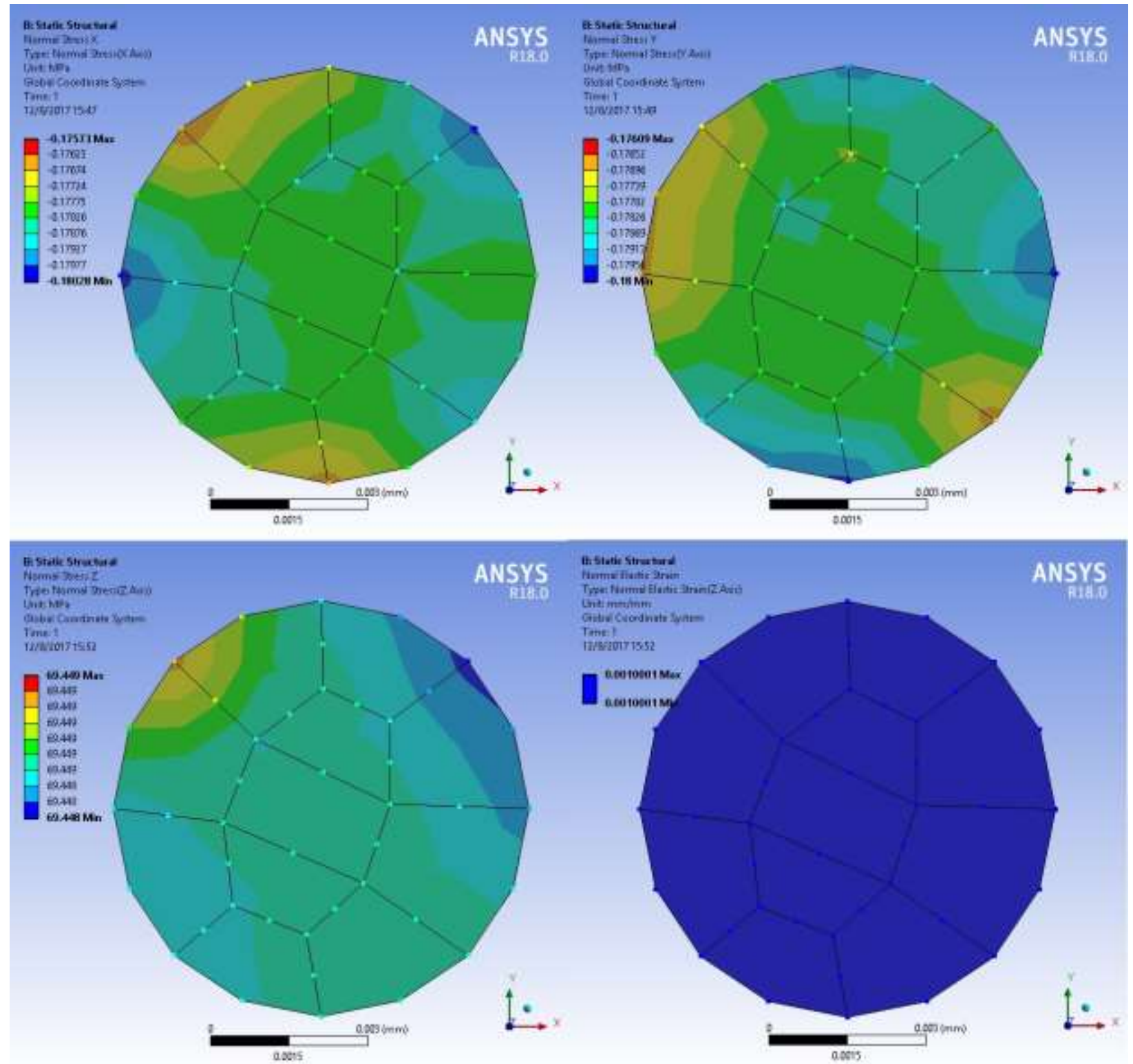

Figure 14 - ANSYS results contour for $\sigma_{x}, \sigma_{y}, \sigma_{z}$ and $\varepsilon_{z}$

It can be concluded from (26) that the contribution of absolute value of optoelastic effect is almost one-sixth of total wavelength shift. Obviously, it is comparable to strain effect and must be taken into consideration for any reliable wavelength shift calculation.

Nevertheless, this procedure is more reliable than the calculation led to the value of $1.2 \mathrm{~nm} / 1000 \mu \varepsilon$, because unlike the theoretical calculation which deliberates just the core of fiber, the FEM model includes both core and cladding, simulates their interaction, and considers 
their effect on each other. The value of $\left(\Delta \lambda_{B}\right)_{y}$ should be the same as $\left(\Delta \lambda_{B}\right)_{x}$ since both the cross section of fiber and the loading scheme are symmetric about the $\mathrm{X}$ and $\mathrm{Y}$ axes. To evaluate the contribution of refractive index changes and elastic strain in wavelength shift, first and the second term of equation (26) can be calculated separately. Doing so we will have:

$$
\begin{aligned}
& \text { 'n'contribution }=1550 \times-1.9919 \times 10^{-4}=-0.3087 \mathrm{~nm} \quad \text { and } \\
& { }^{\prime} \varepsilon^{\prime} \text { contribution }=1550 \times 0.001=1.55 \mathrm{~nm}
\end{aligned}
$$

Electromagnetic waves travelling in the core of fiber, also, can be simulated by COMSOL using FEM. Figure 15 shows the electric field of light waves confined in the core of a microstructured FBG. However, further calculation of EM is out of the scope of this study.

\section{freq $(20)=2.1269 \mathrm{E} 14 \mathrm{~Hz}$ Slice: Electric field norm $(\mathrm{V} / \mathrm{m})$}

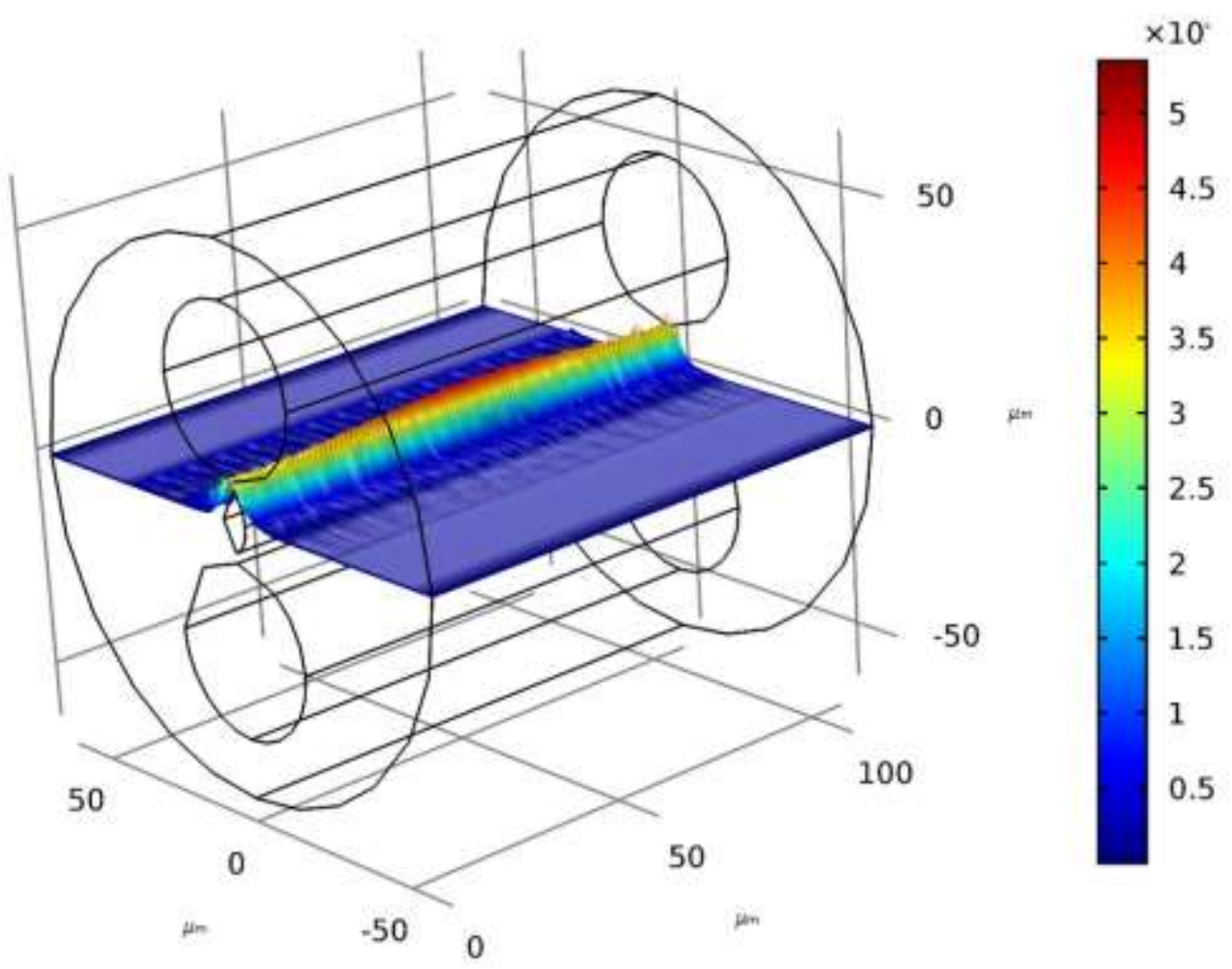

Figure 15 - COMSOL results of the electric field of light waves in a microstructured FBG 


\subsubsection{Force-Displacement Relationship of FBG Under Pressure}

Hydrostatic pressure is a more complex loading sequence which produces three-dimensional stress and strain components in a body, such as an FBG, subjected to it. It is difficult to mathematically calculate the effect of pressure on fiber and FBG similar to what was done to stablish equation (25). Instead, finite element simulation can be used to evaluate the effect of pressure on FBG.

As a simple case, SMF-28 FBG under hydrostatic pressure is simulated by ANSYS first. All geometrical and mechanical properties are exactly the same as used in the previous simulation for the axial strain. The only difference at this time is the applying of a 10 megapascal magnitude hydrostatic pressure on the side surface and end surface of fiber instead of axial strain. Preliminary results are represented in Figure 16. The average wavelength shifts due to 10 megapascal of hydrostatic pressure is $-48.2 \mathrm{pm}$.

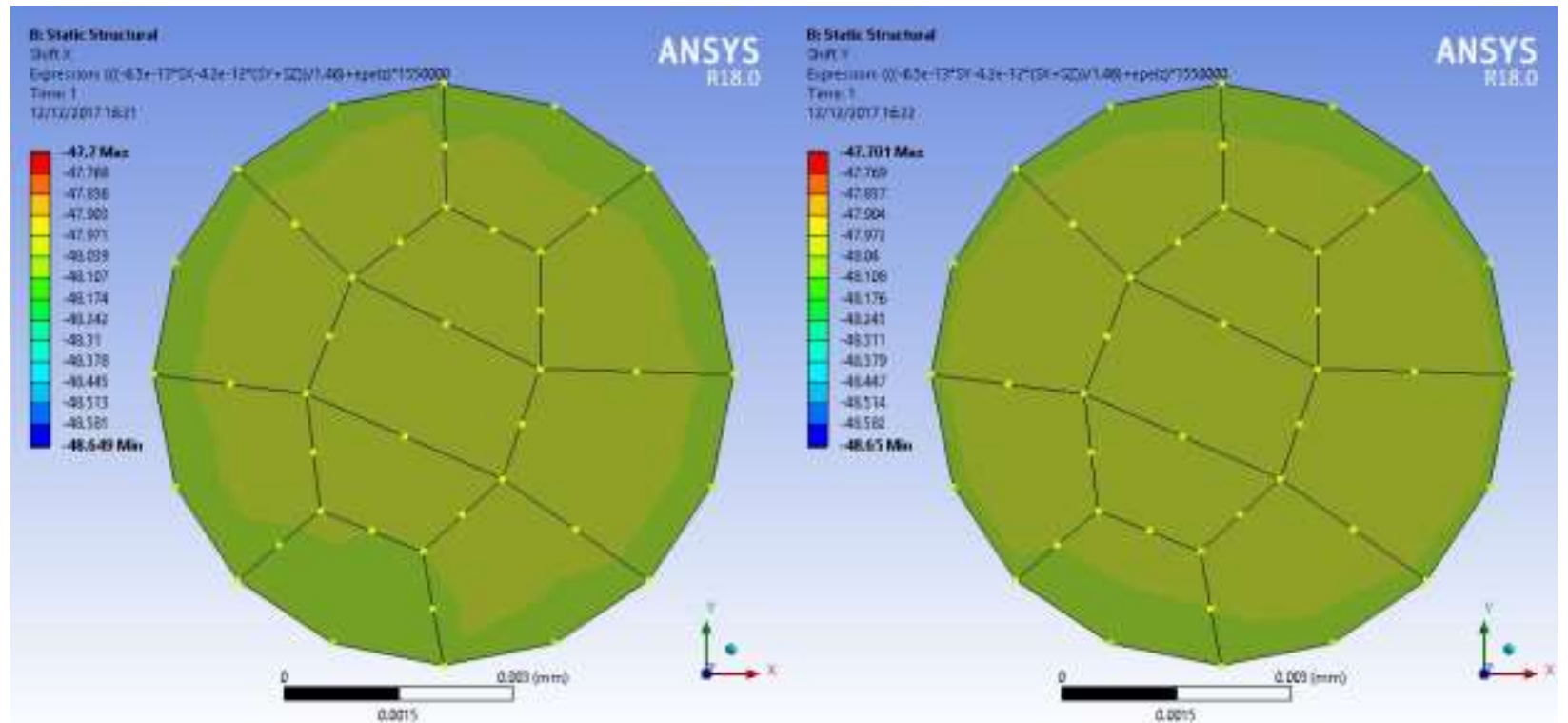

Figure 16 - wavelength shift of SM28 FBG under hydrostatic pressure

Since the geometry of FBG and loading condition are symmetric, the results are exactly the same in the $\mathrm{X}$ and $\mathrm{Y}$ directions. However, the error cased by meshing size induced some variation along the axes of fiber which is negligible compare to the level of pressure. The results in Figure 16 are also in good agreement with the experimental results represented by Chuang $\mathrm{Wu}$ et al. that is -42.3 for 10 megapascals pressure [6]. The minus sign of the final result means that the central 
wavelength of the FBG spectrum shifts to the lower range when the pressure increases. The reason behind negative wavelength shift is when an FBG is squeezed under hydrostatic pressure, its diameter reduces and because of Poisson's effect, its length becomes longer (Figure 17(a)). At the same time, ambient pressure, acting on the end of fiber, tends to decrease its length (Figure 17(b)). The resultant displacement is the superposition of these two displacement components, which is $\delta_{1}-\delta_{2}$ in Figure 17.

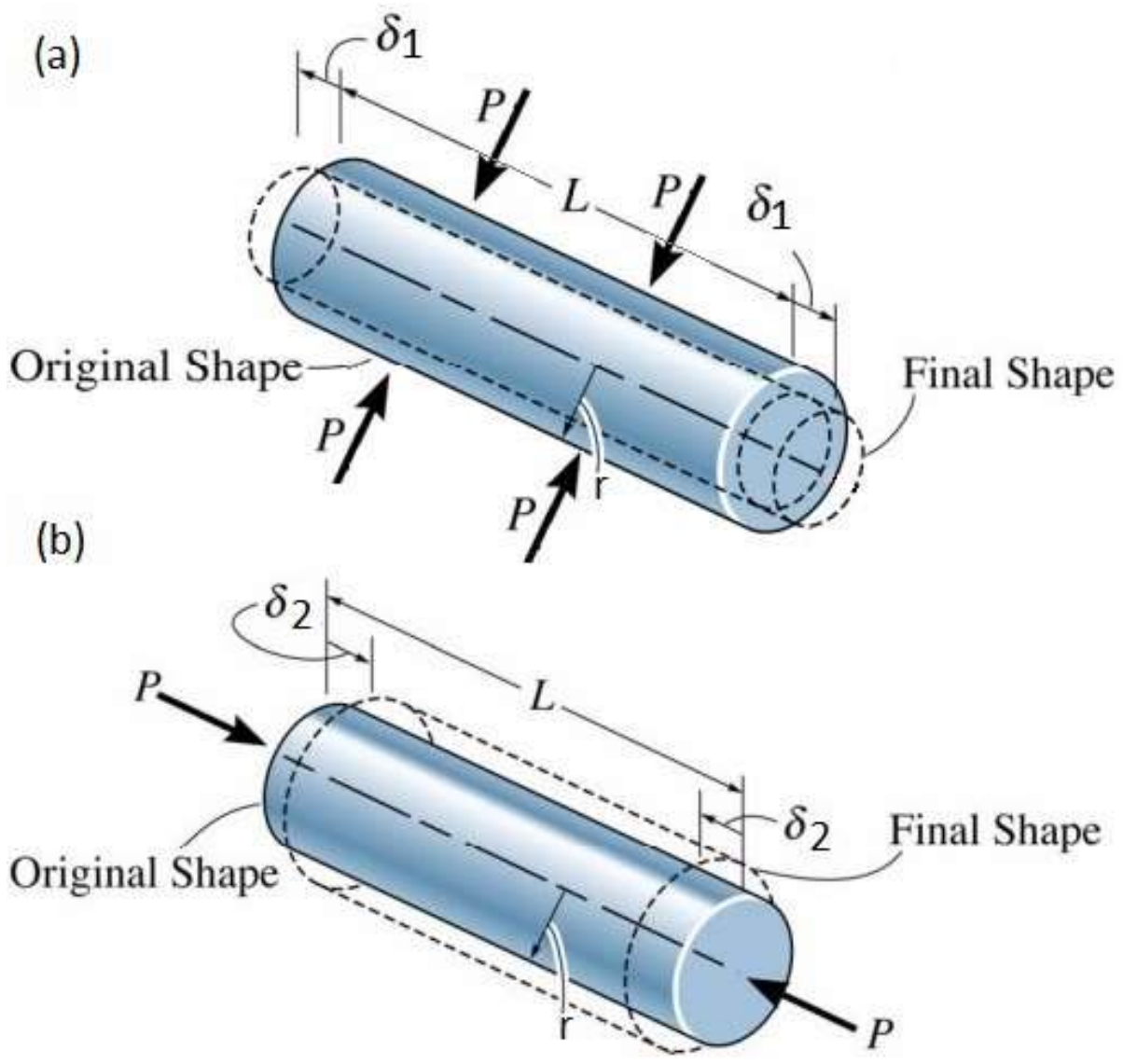

Figure 17 - (a) side pressure increase the length (b) end pressure reduce the length of fiber

Unlike one axis strain measurement applications in which the deformation of FBG is exactly the same as its corresponding mechanical element under investigation, the displacement in FBG experiencing hydrostatic pressure comes from its own deformation which is extremely low. This makes lower pressure difficult to be detected with FBGs. However, by increasing the absolute difference between two displacement components $\left(\left|\delta_{1}-\delta_{2}\right|\right)$ more accurate pressure measurement is feasible with a higher sensitive sensor. To do so, we must analyze these two displacements to find a way to increase their difference. 
For having a better estimation of the effects of side pressure, end pressure and contribution of refractive index and strain change on total wavelength shift, FEM analysis is used and the results are represented in Figure 18 for each of these parameters separately.


Figure 18 - wavelength shifts of SM28 due to (a) side pressure strain (b) side pressure refractive index change, (c) end pressure strain, (d) end pressure refractive index change

As it can be seen in these contours, side pressure causes $72.0 \mathrm{pm}$ wavelength shift from elongation of the FBG (positive strain) and $49.6 \mathrm{pm}$ from changes in the refractive index. End pressure, on the other hand, induces strain related wavelength shift and refractive index change wavelength shift of -212.1 and $42.2 \mathrm{pm}$ respectively. In total, the contribution of these parameters in wavelength shift is $-140.1 \mathrm{pm}$ for strain change (second term of equation (5)) and $91.9 \mathrm{pm}$ for 
refractive index change (first term of equation (5)). Using another method of superposition, we can see that side pressure shifts central wavelength by $121.6 \mathrm{pm}$ (positive shift) and end pressure moves it $-169.8 \mathrm{pm}$ (negative shift).

From the above simulation results, it can be concluded that increasing the deformation of FBG will result in a more sensitive pressure sensor. To increase this deformation as an elastic strain, we must either increase the stress or decrease Young's modulus of materials. Apparently, the material of fiber optic and its mechanical properties such as Young's modulus cannot be changed dramatically. However, stress can be increased by reducing the cross section area of the fiber. Since the overall dimensions of fiber optic are unchangeable, considering void area in its cladding during the manufacturing process becomes the only technique that can be used to decrease the cross sectional area of fiber. Although the holes increase the deformations caused by the side pressure elongation as well, the difference between end and side pressure strain become higher in perforated fiber. Air holes also change the stress distribution inside the core, which makes refractive index change. If the holes placement is unsymmetrical about the origin, stress distribution becomes different in two axes and the total wavelength shift should be calculated separately for each axis. In the other word, the light wave has different travel speed regarding $\mathrm{X}$ and $\mathrm{Y}$ axes and if the light source is considered as linearly polarized, the shift in central wavelength is totally different for two main axes.

Some fiber optics with air holes inside their cladding are available commercially which are used as Polarization Maintain fiber. The philosophy behind the design of these fibers is to achieve different refractive indices for main axes of fiber to transmit the light wave with slightly different speeds in two principal axes. Most of these PM fibers have an elliptical core to boost the refractive index variation. Nevertheless, elliptic shape imposes more asymmetry on the structure of fiber and increase the variation of stress components. To evaluate the effect of pressure on FBGs made on the fiber with voids in their cladding which is also called Microstructured fiber, two different types of them are simulated by ANSYS. The first type has two small holes along its core's minor axis and the second type has two large holes along its core's major axis. The geometry of these two FBGs is shown in Figure 19. The first fiber used for FBG has the minor and major axes of 2.78 and $7.57 \mu \mathrm{m}$ respectively. The cladding diameter of this fiber is $125 \mu \mathrm{m}$ and has two $10.5 \mu \mathrm{m}$ 
diameter voids inside it. The second one has the minor and major axis of 7 and $13.4 \mu \mathrm{m}$ respectively. The cladding diameter of this fiber is also125 $\mu \mathrm{m}$ and has two $40 \mu \mathrm{m}$ diameter voids.

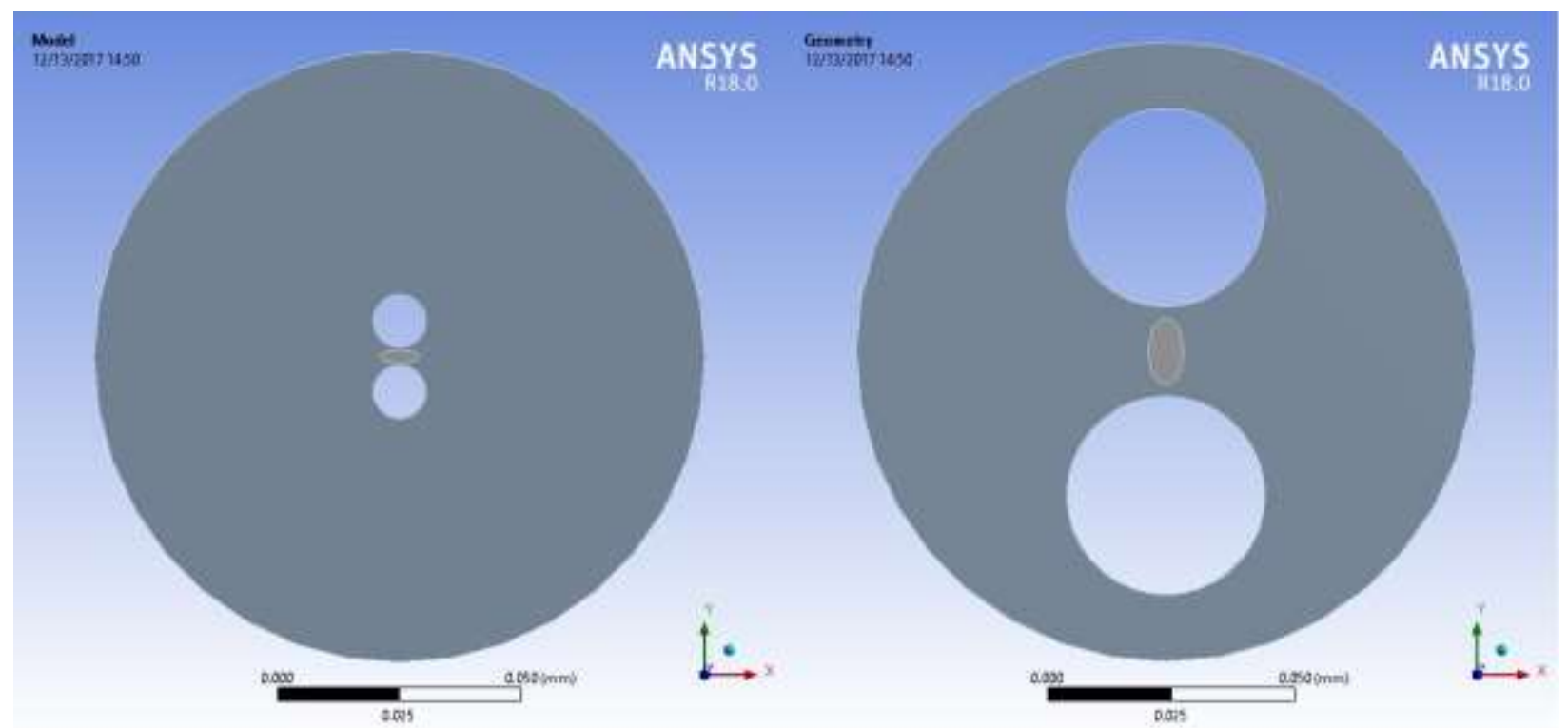

Figure 19 - geometry of two FBGs used for simulation

The results show that not only the values of stress and strain components become different in each axis, but also stress distribution over the core becomes inconstant, which means every single point of the core has its own distinct stress value. As a result, according to equations (17) we have different refractive indices in every single point of the core area. Consequently, for a highly precise simulation, we need to combine electromagnetic wave propagation calculation with mechanical evaluation of stress and strain to calculate the state of light wave according to resultant mechanical stress at each node. Unfortunately, such simulation procedure is extremely complicated and takes a long time to solve even with today's powerful computers. To avoid this complexity, we can simplify the calculation by considering the average value of refractive index change over the FBG's core as a single value. Figure 20 depicts total wavelength shifts caused by 10 megapascal of hydrostatic pressure in small holes fiber. The average shift of central wavelength is $60.1 \mathrm{pm}$ backward in the $\mathrm{X}$ polarization and $11.8 \mathrm{pm}$ forward in the $\mathrm{Y}$ polarization. It should be noted that the averaging value covers total length of the FBG core over its cross section area in order to reduce the effect of meshing error. Figure 21 shows the same results for large holes FBG. In this case, the negative wavelength shift is $79.9 \mathrm{pm}$ again relating to the $\mathrm{X}$ axis and the positive one is $1.7 \mathrm{pm}$ relating to the $\mathrm{Y}$ axis of polarization. 


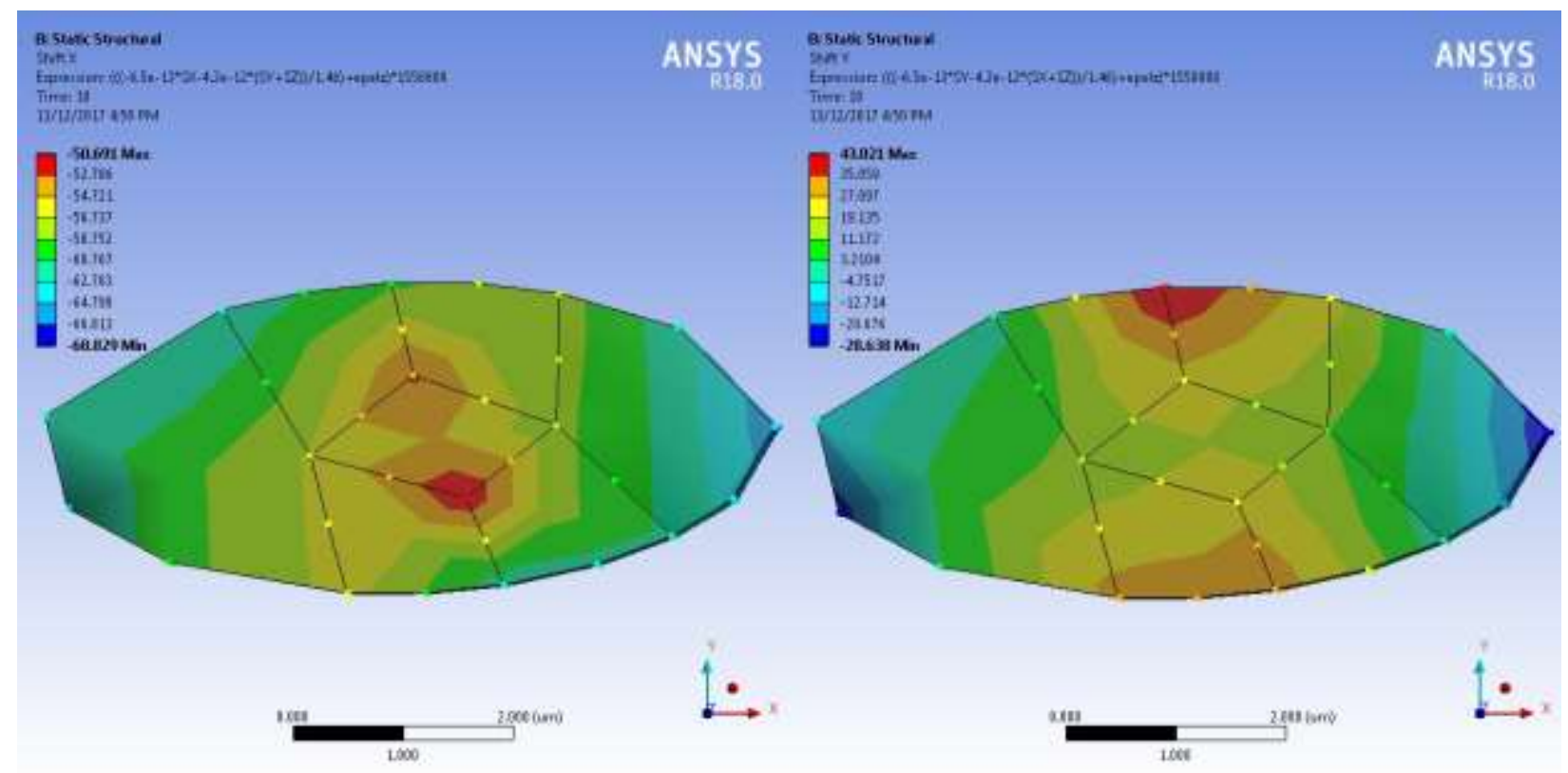

Figure 20 - total wavelength shifts in small hole FBG

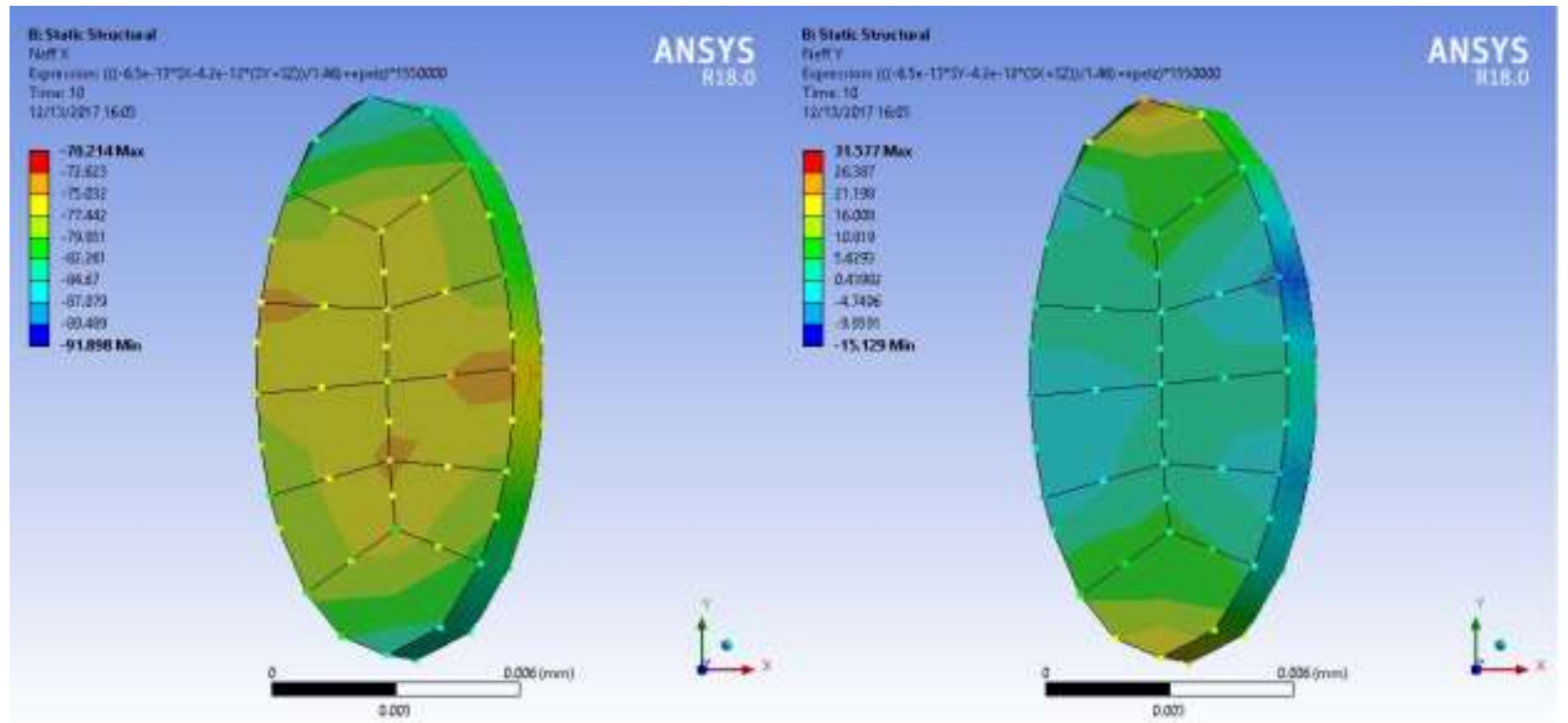

Figure 21 - total wavelength shifts in large hole FBG

The effects of side and end pressure, as well as the contribution of strain and refractive index change to the wavelength shift, are also calculated for both types of FBGs in this simulation. For small holes fiber FBG, side pressure shifts central wavelength of the $\mathrm{X}$ polarization $110.4 \mathrm{pm}$ forward which consists of $72.4 \mathrm{pm}$ from strain change and $37.9 \mathrm{pm}$ from the change in refractive index. At the same time, end pressure induces 170.5 backward shift in central wavelength with the values of $-213.1 \mathrm{pm}$ due to strain and $42.6 \mathrm{pm}$ because of the refractive index change. In order to give a better overview of the simulation results, all outcomes are summarized in Table 2. 


\begin{tabular}{|c|c|c|c|c|c|c|c|c|}
\hline & \multicolumn{4}{|c|}{$\begin{array}{l}\text { Wavelength Shift Due To Side and End } \\
\text { Pressure }\end{array}$} & \multicolumn{4}{|c|}{$\begin{array}{l}\text { Strain and Refractive Index Change Contribution } \\
\text { in Wavelength Shift }\end{array}$} \\
\hline & & SMF28 & $\begin{array}{l}\text { Small } \\
\text { Hole }\end{array}$ & $\begin{array}{l}\text { Large } \\
\text { Hole }\end{array}$ & 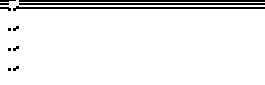 & SMF28 & $\begin{array}{c}\text { Small } \\
\text { Hole }\end{array}$ & $\begin{array}{c}\text { Large } \\
\text { Hole }\end{array}$ \\
\hline \multirow{3}{*}{ 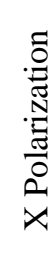 } & $\begin{array}{l}\text { side pressure } \\
\text { effect }\end{array}$ & 12.16 & 11.04 & 12.73 & $\begin{array}{l}\text { strain change } \\
\text { Contribution }\end{array}$ & -14.01 & -14.06 & -16.87 \\
\hline & $\begin{array}{l}\text { end pressure } \\
\text { effect }\end{array}$ & -16.99 & -17.05 & -20.46 & $\begin{array}{c}\text { refractive index } \\
\text { change } \\
\text { Contribution }\end{array}$ & 9.18 & 8.05 & 9.13 \\
\hline & Total & -4.83 & -6.01 & -7.73 & Total & -4.83 & -6.01 & -7.73 \\
\hline \multirow{3}{*}{ 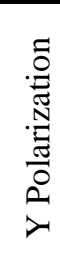 } & $\begin{array}{l}\text { side pressure } \\
\text { effect }\end{array}$ & \multirow{3}{*}{$\begin{array}{l}\text { same as } \\
\text { above }\end{array}$} & 18.24 & 20.62 & $\begin{array}{l}\text { strain change } \\
\text { Contribution }\end{array}$ & \multirow{3}{*}{$\begin{array}{l}\text { same as } \\
\text { above }\end{array}$} & -14.06 & -16.87 \\
\hline & $\begin{array}{l}\text { end pressure } \\
\text { effect }\end{array}$ & & -17.06 & -20.46 & $\begin{array}{l}\because \text { refractive index } \\
\because \text { change } \\
\because \text { Contribution }\end{array}$ & & 15.24 & 17.03 \\
\hline & Total & & 1.18 & 0.16 & Total & & 1.18 & 0.16 \\
\hline
\end{tabular}

Table 2 - simulation results summary, wavelength shift due to pressure

In this table, the wavelength shift values are represented for two main polarization axes. In each polarization axis, the contribution of FBG period change (strain) and refractive index change effect due to pressure change are shown in the right part of the table. To evaluate the amount of total wavelength shift caused by side or end pressure, strain related shift and refractive index change shift are added together in each case and shown in the left half of the table. As it can be seen, for the large holes fiber a total of $-7.73 \mathrm{pm} / \mathrm{MPa}$ wavelength shift is obtained which consists of $12.73 \mathrm{pm} / \mathrm{MPa}$ from side pressure and $-20.46 \mathrm{pm} / \mathrm{MPa}$ from end pressure respectively. On the other hand, we can see that period change (strain) causes central wavelength shift of $-16.87 \mathrm{pm} / \mathrm{MPa}$ while refractive index change causes $9.13 \mathrm{pm} / \mathrm{MPa}$ wavelength shift.

\subsubsection{Effect of Fiber Optic Cross Sectional Dimension and Setup Configuration}

To study the effect of cross sectional dimensions of FBG fiber on its pressure sensitivity, fiber with different cross sectional parameters such as core diameters or holes position is simulated. From the analysis of this calculation outcomes, some improvement can be suggested for a higher sensitive pressure sensor. In another word, simulation can be used as a design tool for high performance FBG pressure sensor.

The results of different cross section simulation show that as the aspect ratio (fast axis diameter/slow axis diameter) of the elliptical core increases, the sensitivity of the slow axis to the pressure decrease while the fast axis remains almost at the same sensitivity. However, the 
birefringence effect that represents the separation of two central wavelengths, become higher in larger aspect ratios. This phenomenon is illustrated in Figure 22.

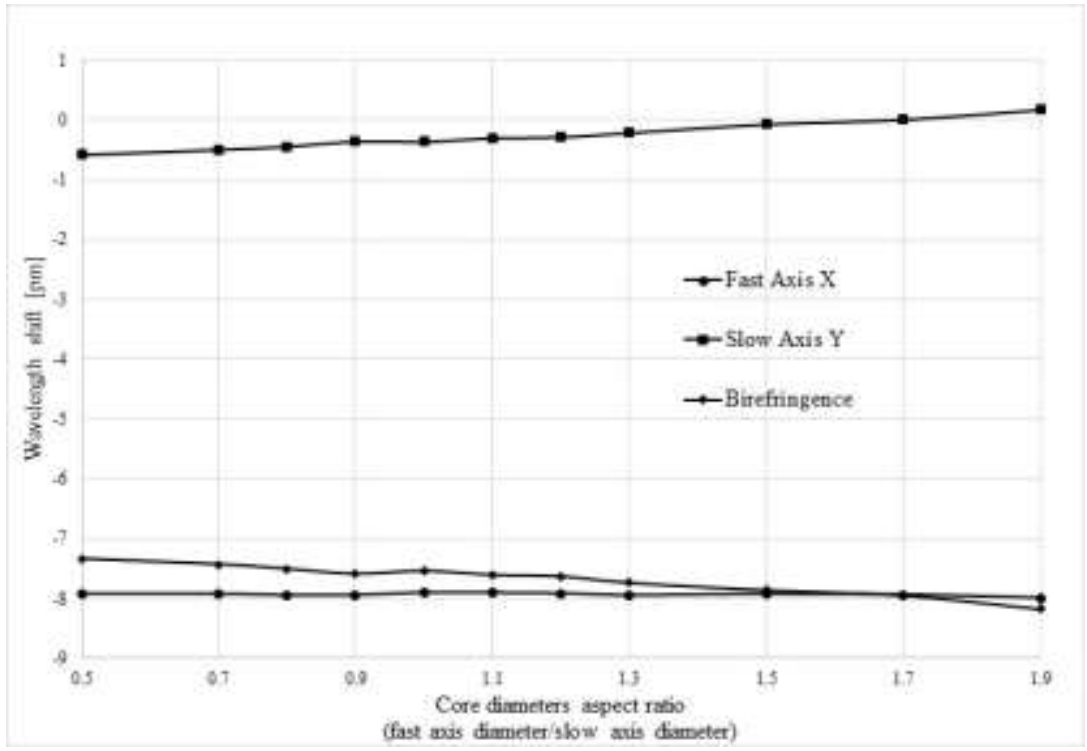

Figure 22 - the effect of core aspect ratio on central wavelength shift

Figure 23 shows the effect of holes' diameter on wavelength shift. As it can be seen in this figure, both axes become more sensitive to pressure change when the diameter of holes' increases. This also causes more birefringence in larger holes' size. Figure 24 depicts the effect of holes distance from the core on wavelength shift. The sensitivity reduces when the holes placed further from the core. This reduction is more obvious in the fast axis. Nevertheless, the birefringence effect becomes higher in more separated side holes' fiber.

The other parameter that has significant influences on the FBG pressure measurements result is the configuration of the sensor, the way that an FBG pressure sensor is terminated and placed in a pressure chamber. Three different configurations are studied theoretically and the results are compared with experimental data. The schematics of these three arrangements are shown in Figure 25. In the configuration shown as Type A, the microstructured fiber containing FBG is blocked by splicing a SMF-28 fiber optic at both ends. 


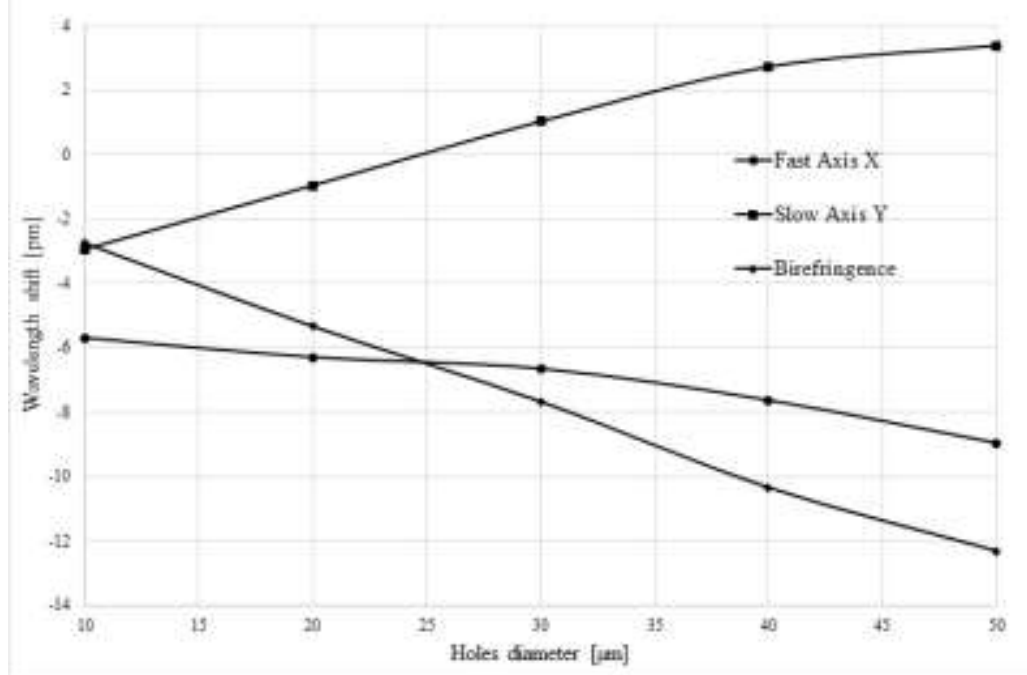

Figure 23 - effect of holes diameter on central wavelength shift

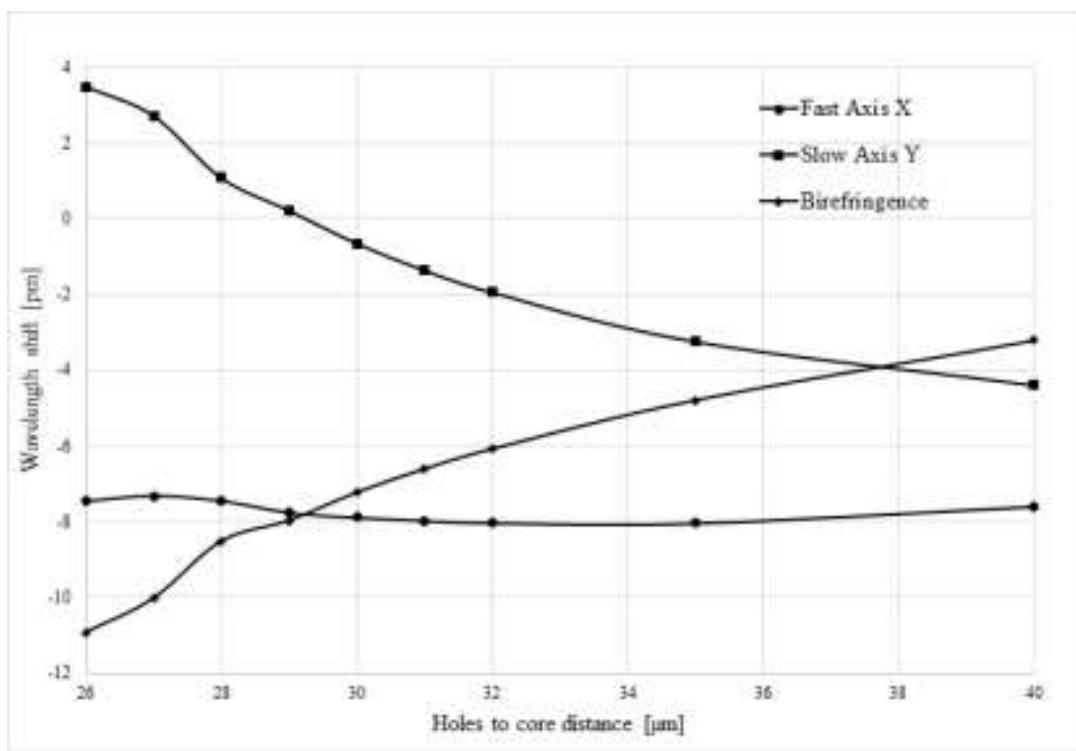

Figure 24 - distance of holes from the core effect on central wavelength shift

One end of the sensor is exposed to pressure in the pressure chamber (left end in Figure 25) and the other end is connected to a measuring fiber cable carrying optical signals out of the chamber with a fiber feedthrough. The simulation results described in the previous section, with the negative wavelength shift, are all calculated based on this configuration.

In Type B configuration, both ends of the FBG are blocked by SMF-28 fiber and left outside of the pressure chamber. This setup prevents the ends of sensor from the applied pressure; so the FBG sensor only experiences side pressure. 


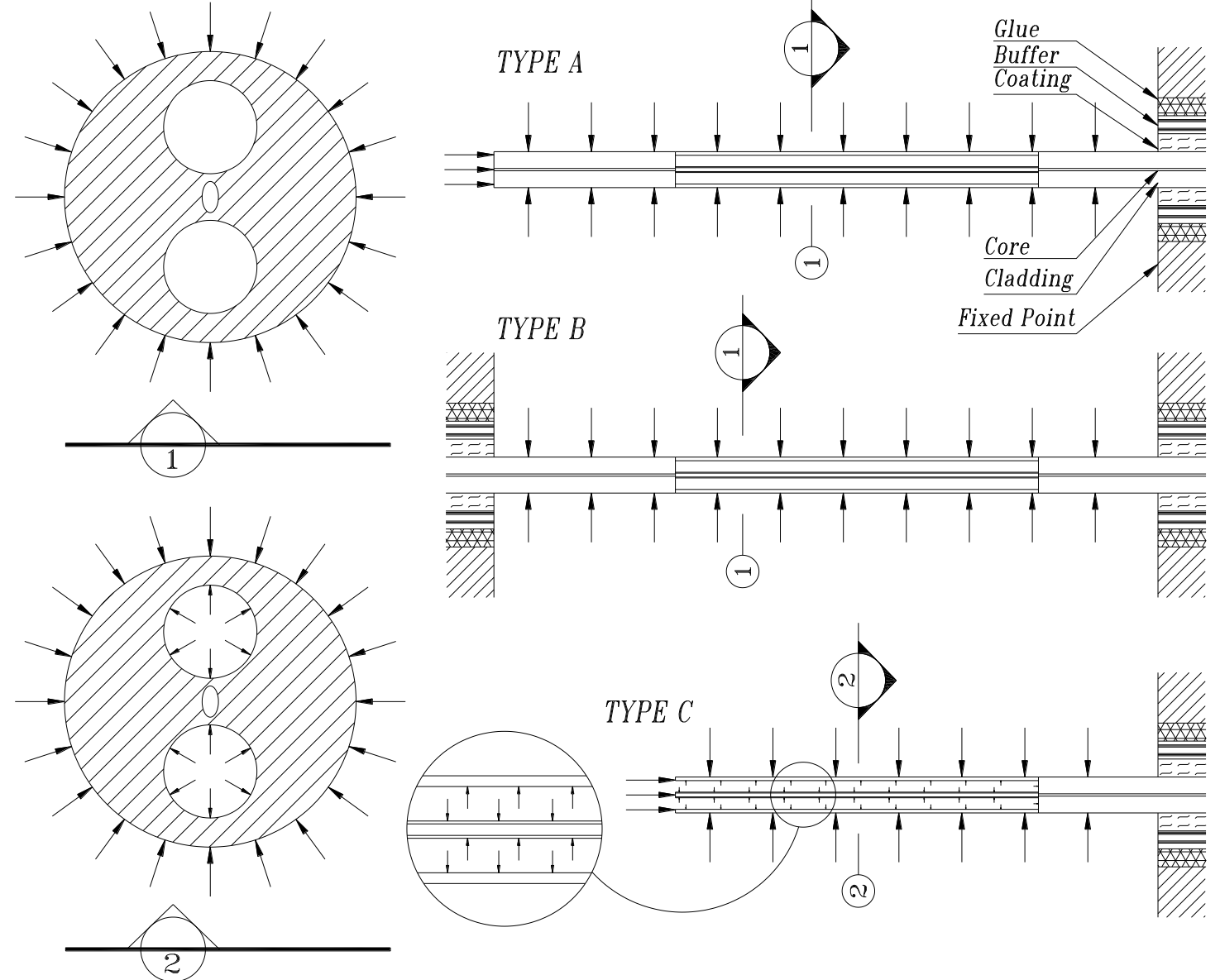

Figure 25 - three different types of FBG pressure sensor setup

Due to the flexibility of connections at both ends, this sensor becomes elongated when the pressure increases. Simulation result of this setup retunes two positive values of central wavelength shift, $8.748 \mathrm{pm} / \mathrm{MPa}$ for fast axes and $16.897 \mathrm{pm} / \mathrm{MPa}$ for slow axis, which means applying only side pressure causes elongation of the fiber due to Poisson's effect. I obtained different calculated values, but similar wavelength shifts directions as compare to Chen et al. experiment [7]. Obviously, the difference in values originated from the difference between dimensions and properties of their fiber and that of my fiber. However, this configuration of sensor setup shows a higher sensitivity than other two types.

The only different between the last configuration, Type $\mathrm{C}$, and Type $\mathrm{A}$ is that in Type $\mathrm{C}$ one end of microstructured fiber is open and the gas can flow inside its holes. In this case, both peaks shift to lower wavelength with the same trend, indicating lower birefringence. The fast and slow axis 
wavelength shift is $-4.69 \mathrm{pm} / \mathrm{MPa}$ and $-4.66 \mathrm{pm} / \mathrm{MPa}$ respectively. An experiment was conducted for this situation and the results were evaluated which were in agree with the simulation. 


\section{Pressure and Temperature Measurement Experiment}

To study the behaviour of microstructured FBG sensors under hydrostatic pressure in different ambient temperatures, I used two different types of fiber both have two voids in their cladding next to their core. Figure 26 shows the cross section of the fibers used for FBG writing which are similar to what simulated by FEM in previous section. The first fiber is an SPF-1550 from Corning Corp. The SPF-1550 fiber has an elliptical core with the major and minor axis of 7.57 and 2.78 microns respectively, two small holes in its cladding have the diameter of $10.5 \mu \mathrm{m}$, and the total diameter of 125 microns. The second fiber has larger holes with a 40 micron diameter and bigger core with a $13.4 \mu \mathrm{m}$ major axis length and $7 \mu \mathrm{m}$ minor axis length.

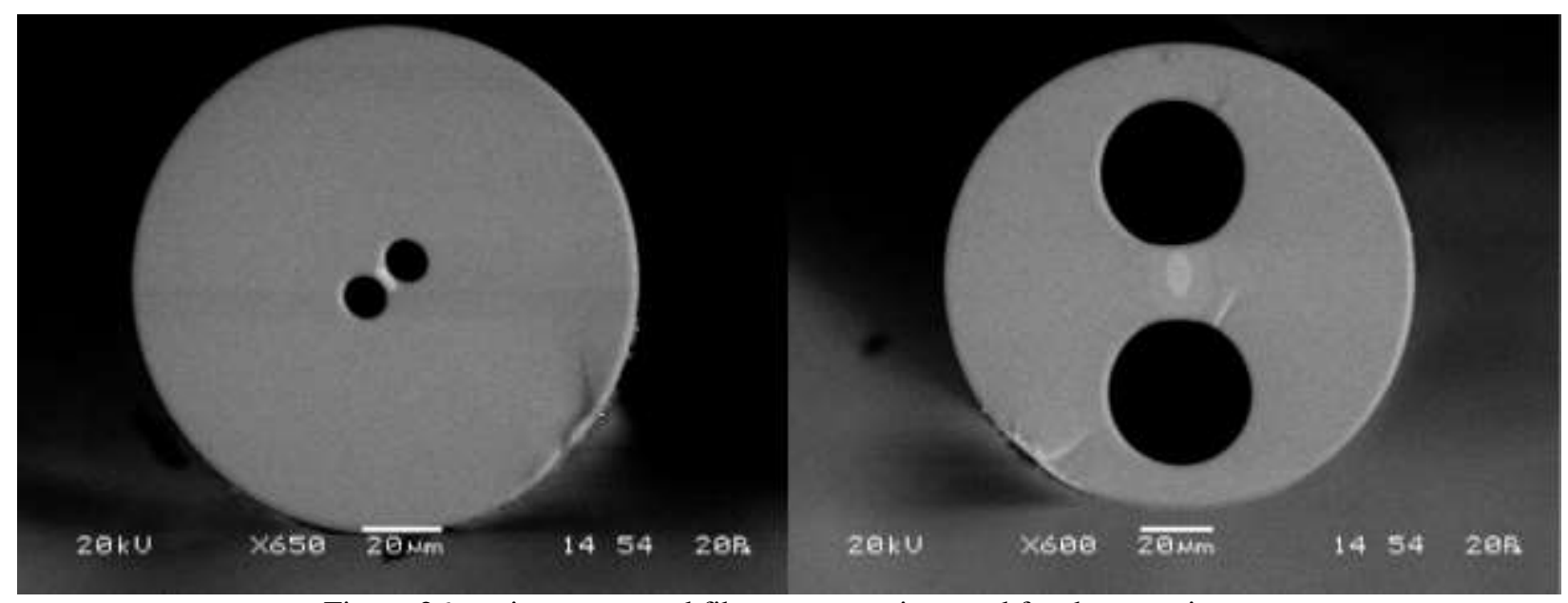

Figure 26 - microstructured fiber cross section used for the experiment

\subsection{Experiment Setup}

Since the physical dimensions of the FBG sensor are relatively small, it can be easily inserted inside a $1 / 4$ inch stainless steel tube. I used a fiber feedthrough to seal the end of the pressure tube and FBG sensor was spliced to this feedthrough. The pressure supplied by a Nitrogen or Hydrogen cylinder and controlled by a conventional gas pressure regulator. The regulator which its outlet was connected to the experiments tube reduced the gas pressure to a desired value. The tube then was put inside a temperature chamber with controlled ambient temperature. Figure 27 shows the whole setup with its parts as following:

1. Pressure regulator

2. Temperature chamber 
3. Nitrogen gas cylinder

4. The tube containing microstructured FBG sensor

5. Fiber optic cable

6. Tunable laser

7. Optical circulator

8. Thermocouple thermometer

9. Controlling and data collecting computer

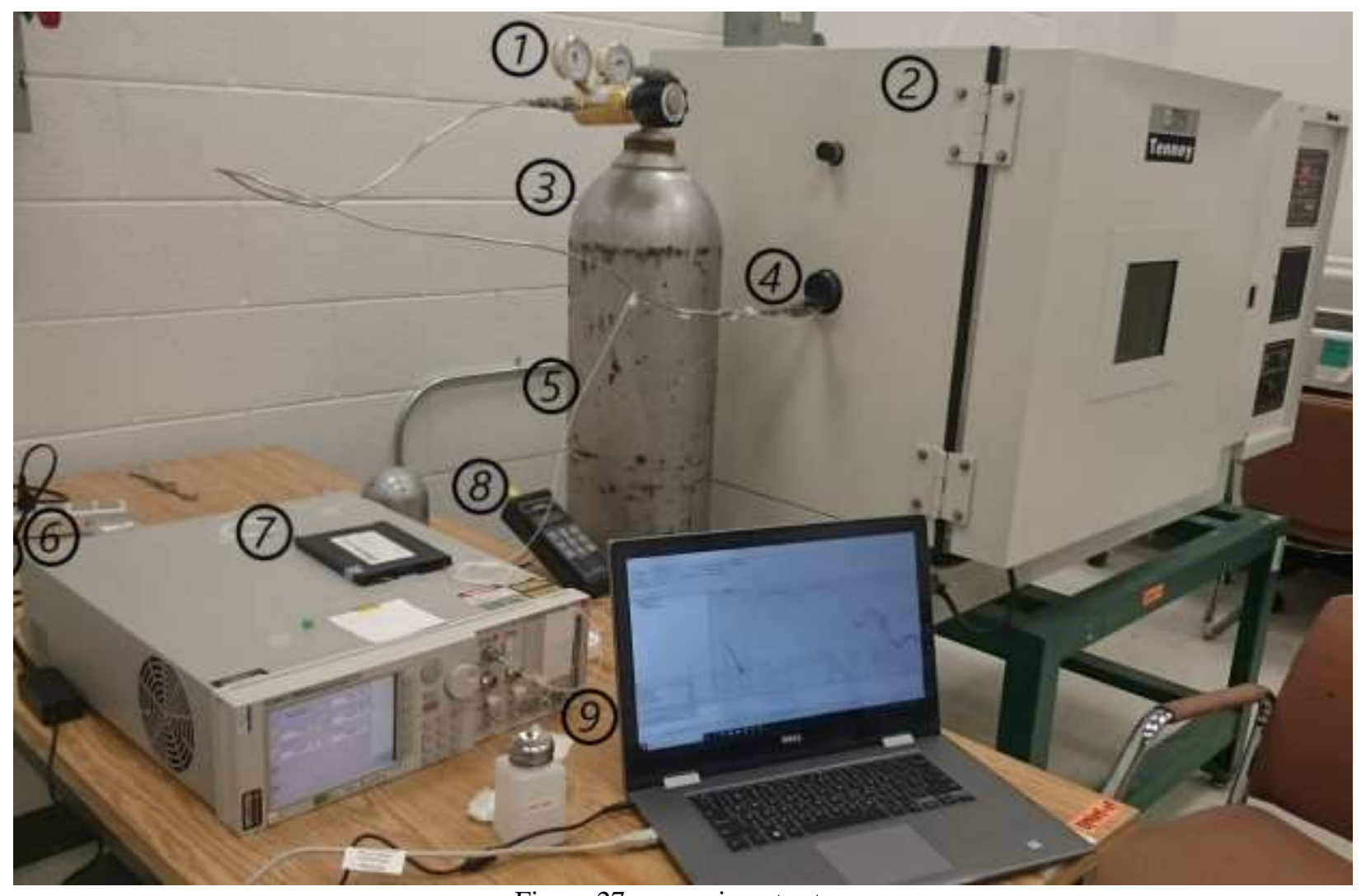

Figure 27 - experiment setup

\subsection{Devices and Instruments}

The main measuring instrument used in my experiment was a tunable laser source (Agilent Model 8164A Lightwave Measurement System). This main frame contains a tunable laser source which can sweep light wavelength range at a resolution of 0.2 picometers. It has a InGaAs power meter which can measure the power of light from $-80 \mathrm{dBm}$ to $+10 \mathrm{dBm}$ with a peak to peak noise less than 20 picowatts. The output spectra of this power meter were saved in Microsoft Excel sheets for further data processing. 
The Tenney temperature chamber was used to set the temperature to a certain level with a resolution of $0.1{ }^{\circ} \mathrm{C}$. Tenny shows its inside chamber mean temperature on a front display; however, I used a thermocouple attached to the tube containing FBG to measure the temperature separately.

\subsection{Configuration of The FBG Pressure Sensor}

I used two types of FBGs in my experiments: a regular FBG writing on a SPF-1550 fiber and $\pi$-phase shifted FBG drawn on both SPF-1550 and large holes fiber. A $\pi$-phase shifted FBG has a passing band in its transmission spectrum caused by a $\pi$-phase shift inscribed in the middle of the grating in the fabrication process. In other word, a $\pi$-phase shifted FBG comprises two identical FBG with a half period (pi) phase deference in their refractive indices modulation. The $\pi$-phase shift induces a narrow bandpass resonance at the middle of its transmission spectra of the FBG. This resonance peak can be as narrow as few picometers which makes the FBG a highly precise wavelength measurement device. Figure 28 illustrates raw spectra of two different FBGs, one is standard and another is $\pi$-phase shifted. As it can be seen, the standard FBG has a significantly higher $3 \mathrm{~dB}$ linewidth than $\pi$-phase shifted type. This narrow linewidth increases the sensitivity of FBG as we will see in experimental results. Figure 29 depicts large holes fiber's $\pi$-phase shifted spectra with a middle notch linewidth of 12.8 picometers. The two separated peaks in large hole fiber FBG's spectra are related to two axes of light source polarization as it mentioned theoretically in previous sections. Total $3 \mathrm{~dB}$ linewidth of this FBG is 237 picometers.

It is explained in section 3.3.2 that the configuration of the FBG sensor has a significant effect on the pressure measurements. In my experiment, Type A configuration was used in which both ends of microstructured FBG are blocked by simple SMF-28 fiber and one end of the sensor is exposed to pressure. In such a configuration, only one end of sensor was accessable for signal (light wave) transfer and the measurement can only be done in reflection mode. For this reflection mode measurement, an optical circulator was used to send light to the sensor and the reflecting light was measured. Transmission spectra, however, can be measured using Type B setup. I also conducted an experiment to study the behaviour of an open ended microstructured FBG sensor, Type C. 


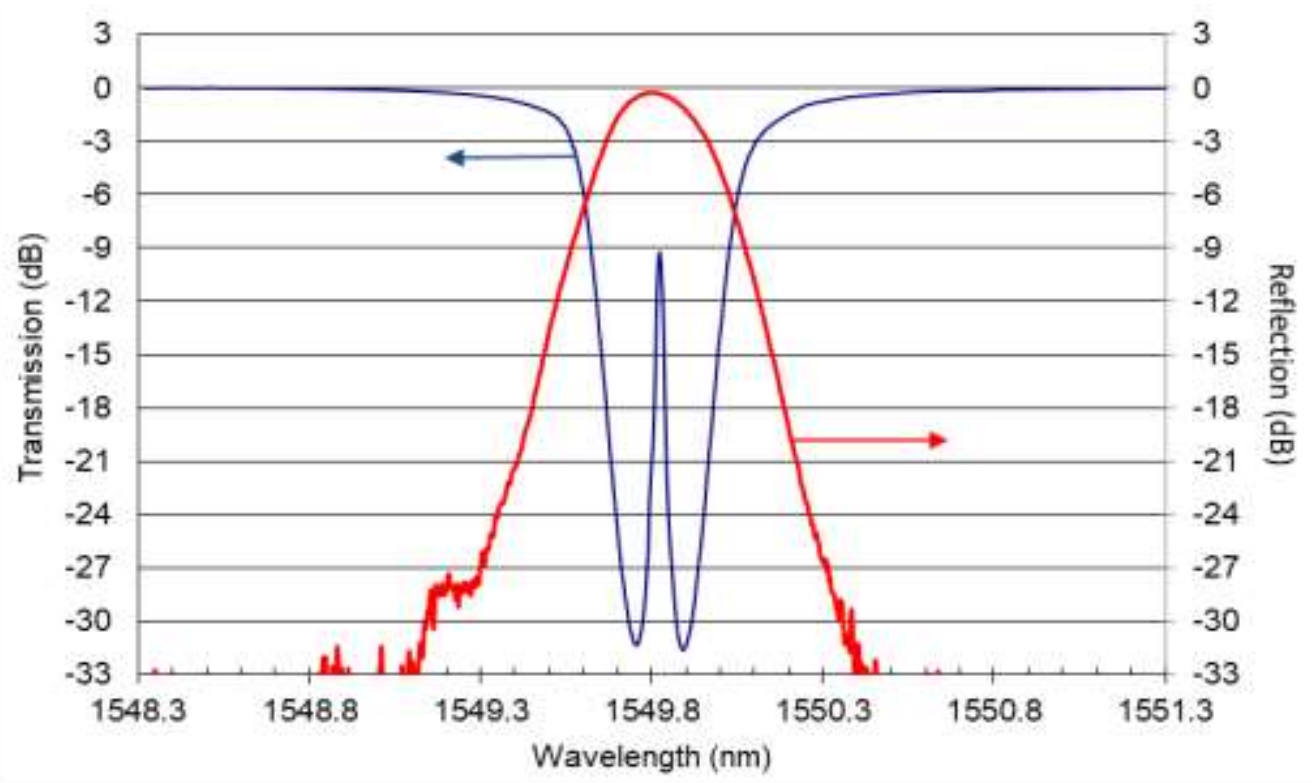

Figure 28 - A reflection spectrum of a typical conventional FBG (red) versus a transmission spectrum of a $\pi$-phase shifted FBG (blue)

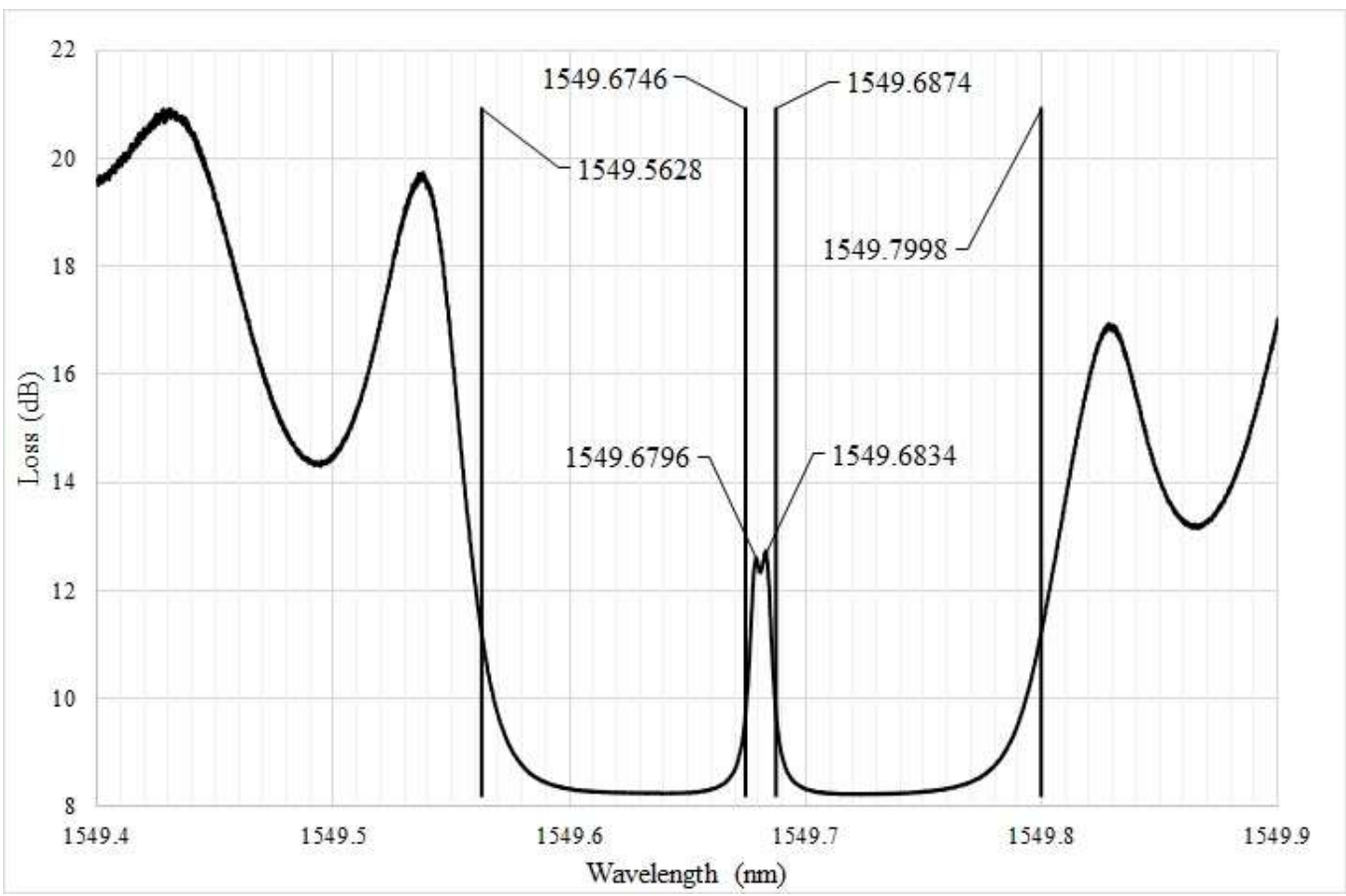

Figure 29 - raw spectra of large hole fiber $\pi$-phase shifted FBG

The total length of my sensor was almost 30 centimetres which included $20 \mathrm{~cm}$ microstructured fiber and 10cm SMF-28 fiber for end capping. Figure 30 shows a schematic diagram of the sensor's connections. 


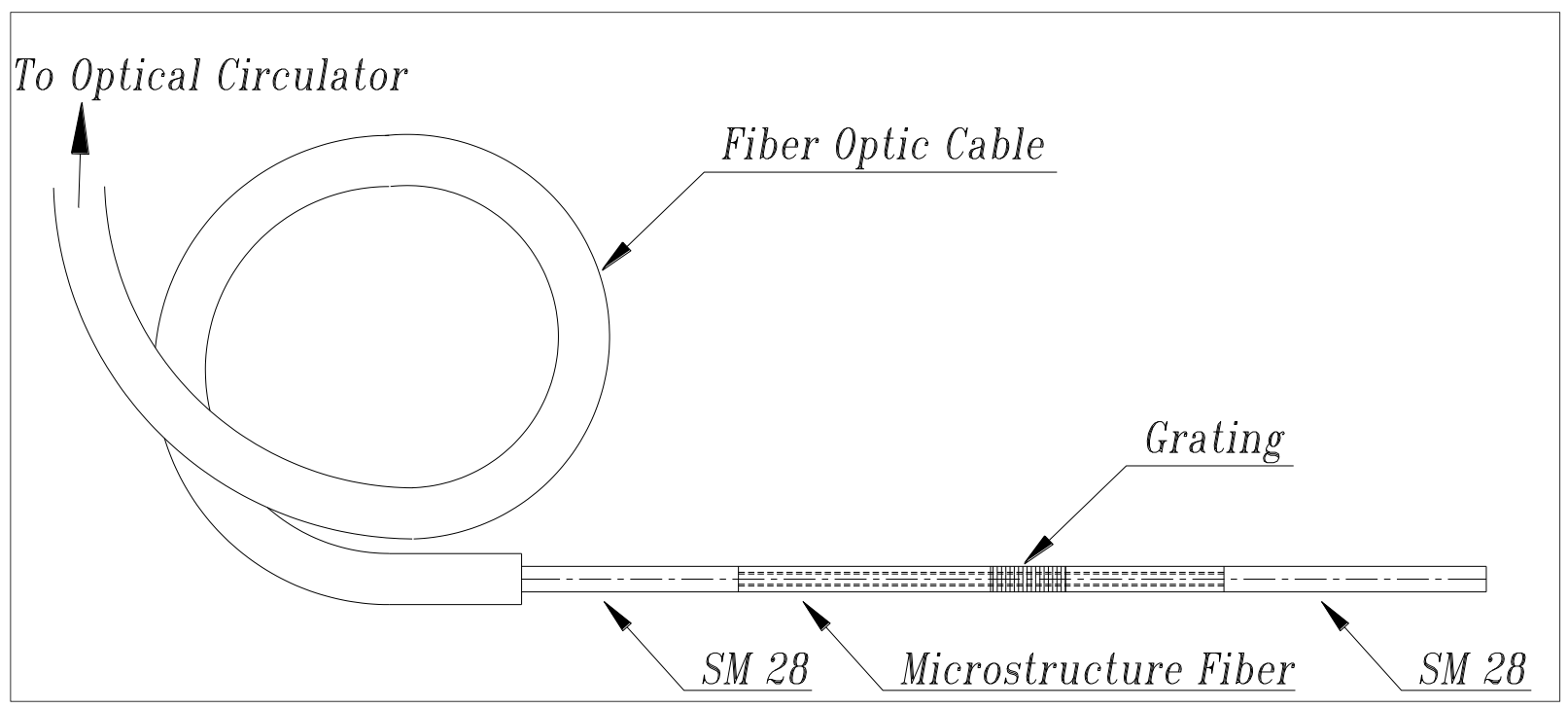

Figure 30 - sensor assembly 


\section{Experimental Results and Relative Simulation Errors}

In this section, I examine all theories explained previously by experiments.

\subsection{Pressure Measurement at Room Temperature}

In this experiment, I measured the response of my three different FBG sensors to the rising pressure at room temperature. The pressure level starts at one atmosphere pressure and increases by the increments of 1 megapascal. Figure 31 shows the spectra shift in a standard and a $\pi$-phase shifted SPF-1550 FBG respectively.
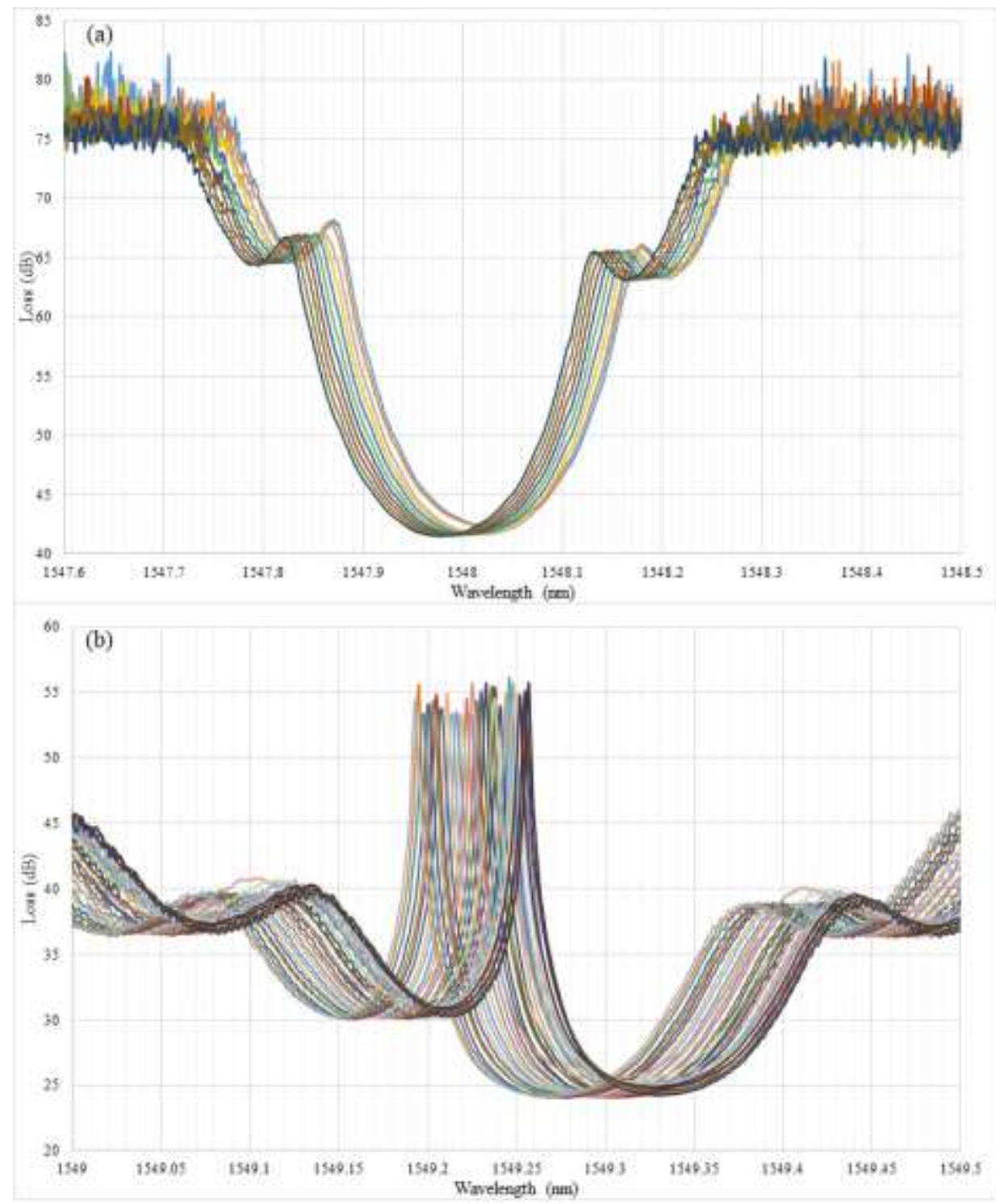

Figure 31 - reflected spectra shift due to rising pressure (a) standard FBG (b) $\pi$-phase shifted FBG 
The result of wavelength shifts versus applied pressure is graphed in Figure 32. As it can be seen, $\pi$-phase shifted FBG shows higher sensitivity to the pressure change. Standard FBG has a sensitivity of $-4.47 \mathrm{pm} / \mathrm{MPa}$ while $\pi$-phase shifted FBG shows $-5.27 \mathrm{pm} / \mathrm{MPa}$. $\pi$-phase shifted FBG also reacts more linearly to pressure change. In fact, more precise measurement with narrow linewidth $\pi$-phase shifted FBG provides more linear pressure measurement. The regression coefficient of determination, $\mathrm{R}^{2}$, for standard and $\pi$-phase shifted FBGs are 0.9966 and 0.9989 respectively. The SPF-1550 FBG spectra contain only one peak even in $\pi$-phase shifted type since this type of fiber is designed to supress one polarization.

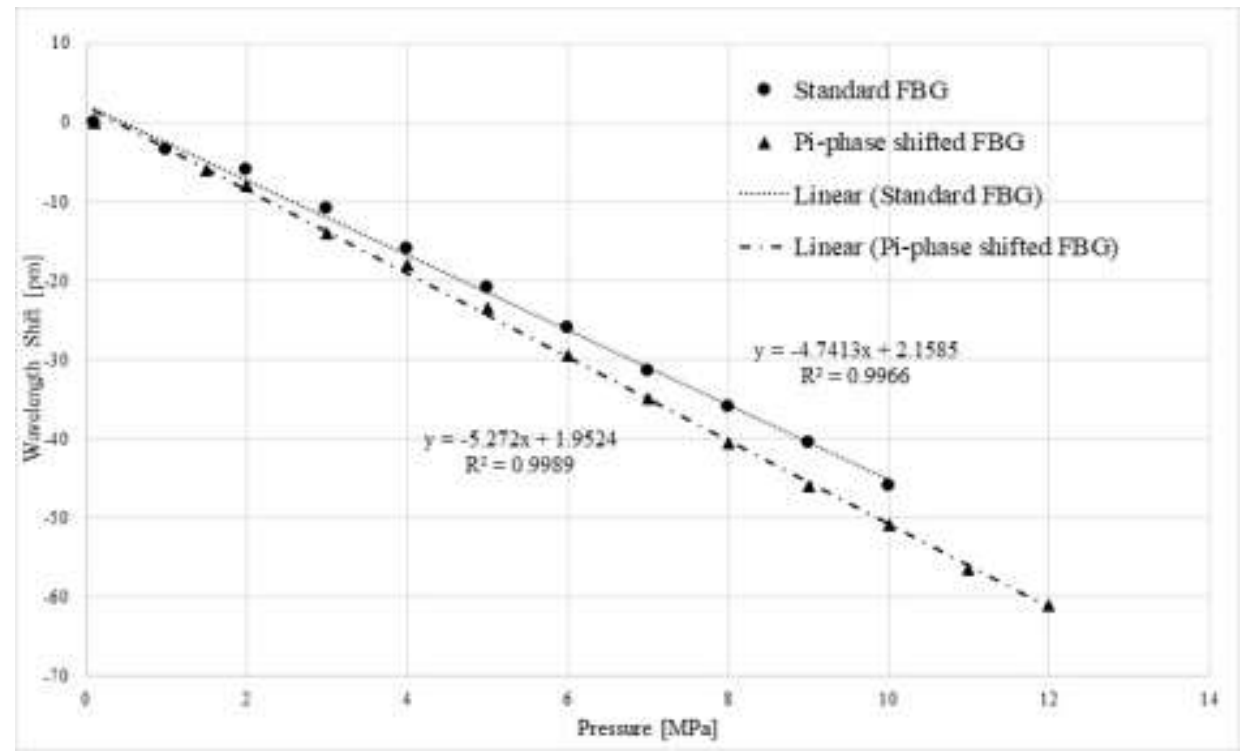

Figure 32 - standard and $\pi$-phase shifted SPF-1550 FBG pressure response

Results of the pressure measurements from a large holes fiber FBG sensor are represented in Figure 33. This FBG has two separated peaks in its spectra and each peak responds to the rising pressure differently. Figure 33(a) shows the spectra change when the pressure is applied and Figure 33(b) represents the pressure responses of two peaks. As it is shown, the fast axis of FBG has a sensitivity of $-6.75 \mathrm{pm} / \mathrm{MPa}$ to pressure change while the slow axes remain at the almost same position during the experiment. This insensitivity to pressure change can also be seen in spectra shifts in Figure 33(a). As the results, the spectral separation between the two peaks can also be used for pressure measurement, which shows a sensitivity of $-6.98 \mathrm{pm} / \mathrm{MPa}$ to pressure change. The regression coefficient of determination for these experimental results was calculated as 0.9964 for both fast axis wavelength shift and peaks difference. 
It should be noted that the reason for the negative wavelength shifts is the mechanical behaviour of the sensor under ambient pressure. This is a Type A configuration according to section 3.3.2, in which the rising pressure causes a shortening in the total length of FBG and consequently a negative wavelength shift reading.
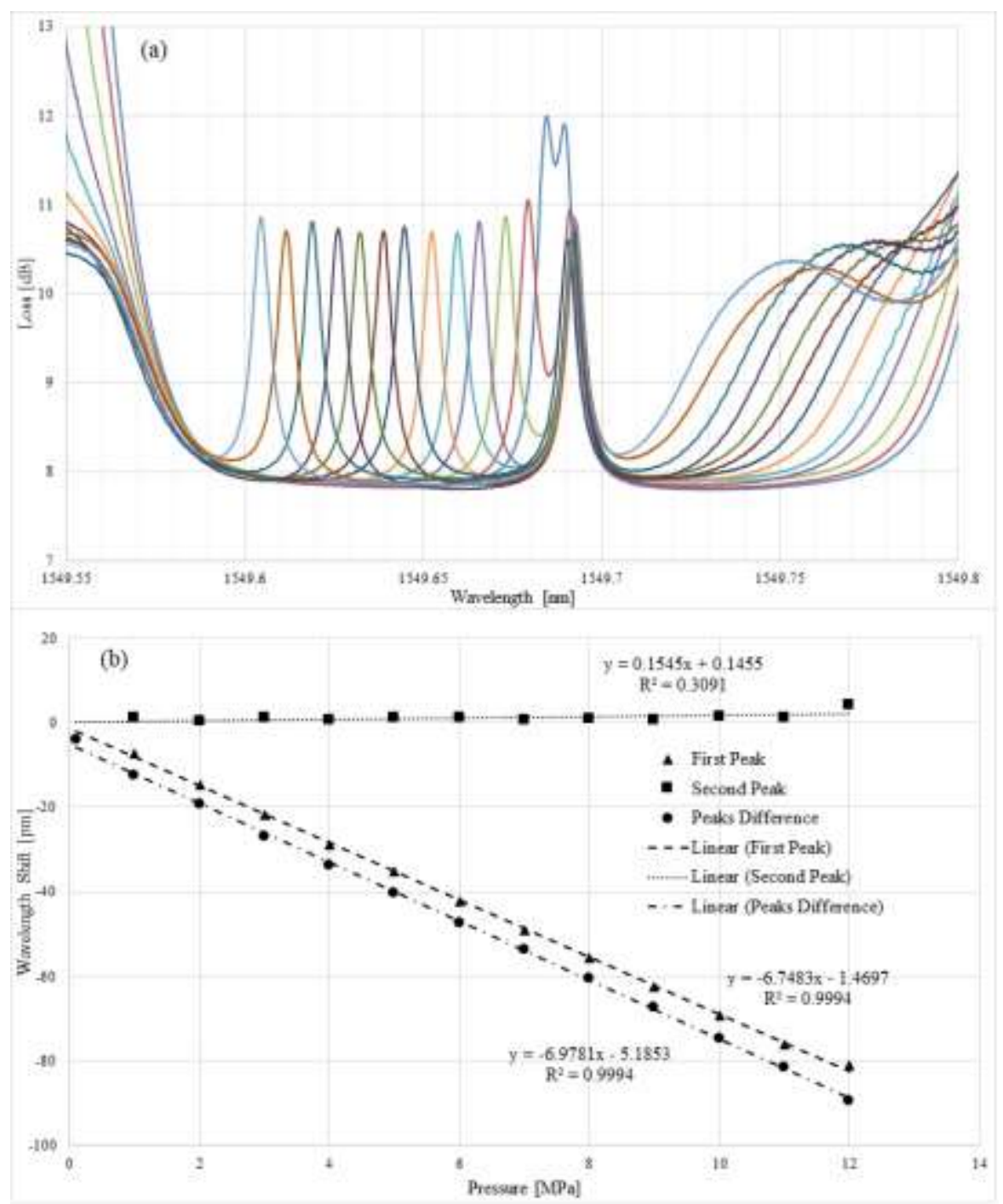

Figure 33 - large hole fiber (a) spectra shift (b) central wavelength shift due to rising pressure

Since the large hole fiber FBG shows more sensitivity to pressure change and both polarization peaks are detectable in the measured spectra, I used this type of FBG for my all other experiments. Also, all the FBGs in remaining experiments are $\pi$-phase shifted, as they are more sensitive to pressure change and return more accurate results. 


\subsection{Temperature Measurement at Room Pressure}

The central wavelength of an FBG shifts when its ambient temperature changes according to equation (8). As it mentioned in section 1.1.3.3, the contribution from refractive index change in this wavelength shift dominates the thermal expansion effect. Therefore, we expect consistent results in this experiment with another type of FBG temperature measurement attempts since stress and strain changes have no significant effect in temperature measurement. Results of my experiment in the atmospheric pressure show that the two peaks move together with the rate of $10.21 \mathrm{pm} /{ }^{\circ} \mathrm{C}$. The experiment is started from zero degree Celsius, and raised to $100^{\circ} \mathrm{C}$ in an intervals of $10^{\circ} \mathrm{C}$. The output spectra for $0,20,40,60,80$, and 100 degrees centigrade are shown in Figure 34(a) and the graph of central wavelength shift due to raising temperature is represented in Figure 34(b). As it can be seen in the graph, the regression coefficient of determination of this experiment outcomes is 0.9993 .
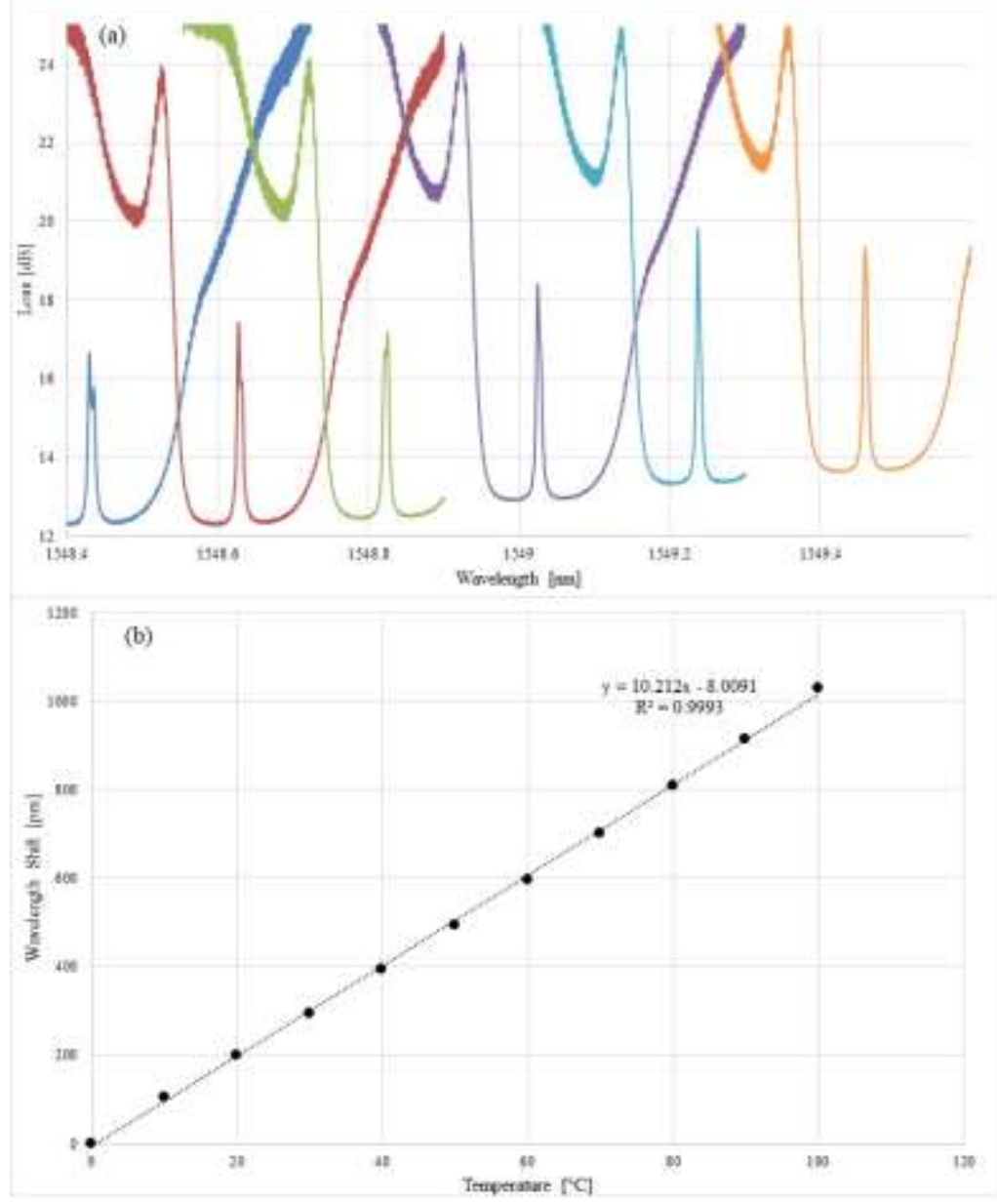

Figure 34 - large hole fiber (a) spectra shift (b) central wavelength shift due to rising temperature 


\subsection{Simultaneous Pressure and Temperature Measurement}

The unique characteristic of microstructured fiber of having two peaks in their reflected (transmitted) spectra with two different rates of sensitivity to pressure and temperature, makes them a precious choice for simultaneous pressure and temperature measurement. For this purpose, the values of the sensitivity matrix, $[\mathrm{K}]$, in equation (14) must be found. To find the values of $[\mathrm{K}]$, pressure measurements in the temperature chamber at the controlled temperatures of $-20^{\circ} \mathrm{C}, 0^{\circ} \mathrm{C}$, and $45^{\circ} \mathrm{C}$ were carried out. Then the wavelength shifts due to temperature change at the controlled pressure of $0.1 \mathrm{MPa}, 5 \mathrm{MPa}, 10 \mathrm{MPa}$, and $12 \mathrm{MPa}$ are plotted out. Figure 35 and Figure 36 show the result of wavelength shift in controlled temperature raising pressure and controlled pressure raising temperature respectively from the two peaks of the microstructured fiber FBG sensor.
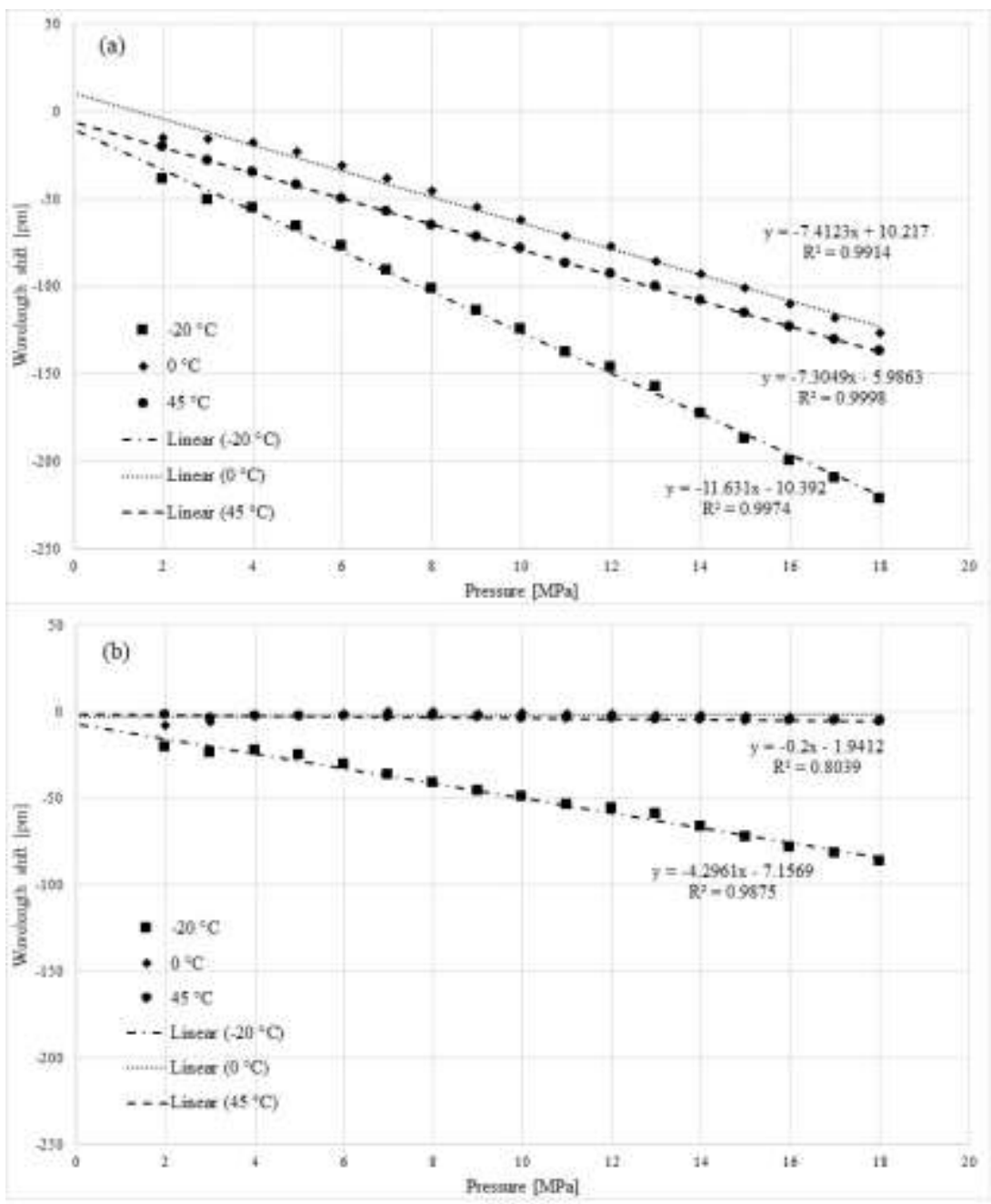

Figure 35 - pressure measurements in 3 different temperature level (a) fast axis (b) slow axis 

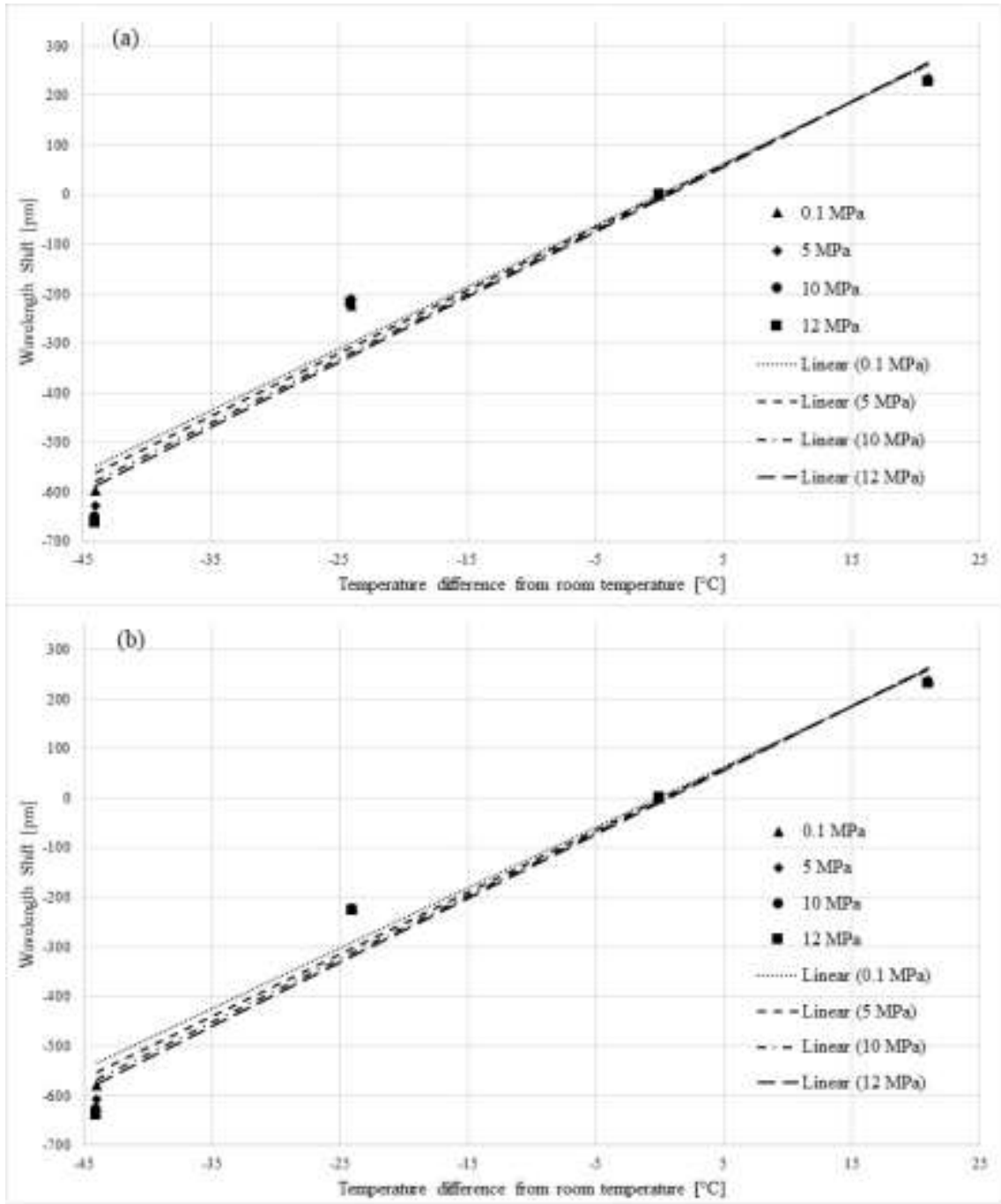

Figure 36 - temperature measurements in 4 different pressure level (a) fast axis (b) slow axis

It should be noted that the values of temperature in the relating graph are the difference between experiment temperature and room temperature. The value of the sensitivity matrix can be extracted from these graphs as follow:

$$
\left[\begin{array}{lll}
\frac{\partial\left(\Delta \lambda_{B}\right)_{x}}{\partial P} & \frac{\partial\left(\Delta \lambda_{B}\right)_{x}}{\partial T} & \frac{\partial^{2}\left(\Delta \lambda_{B}\right)_{x}}{\partial P \partial T} \\
\frac{\partial\left(\Delta \lambda_{B}\right)_{y}}{\partial P} & \frac{\partial\left(\Delta \lambda_{B}\right)_{y}}{\partial T} & \frac{\partial^{2}\left(\Delta \lambda_{B}\right)_{y}}{\partial P \partial T}
\end{array}\right]=\left[\begin{array}{lll}
-7.5508 & 12.4100 & 0.0614 \\
-0.4139 & 12.1550 & 0.0554
\end{array}\right]
$$

where the pressure related wavelength shift is in $\mathrm{pm} / \mathrm{MPa}$, the temperature related wavelength shift is in $\mathrm{pm} /{ }^{\circ} \mathrm{C}$, and the second order wavelength shift is in $\mathrm{pm} / \mathrm{MPa} /{ }^{\circ} \mathrm{C}$. The temperature measurement results are also quite linear with an average regression coefficient of determination, $\mathrm{R}^{2}=0.9684$. 
Having had this sensitivity matrix, we can measure both temperature and pressure at the same time with the microstructured fiber FBG.

\subsection{Time response of Pressure Measurement}

As stated previously, temperature and pressure can be calculated or measured simultaneously when they affect the FBG independently. However, when the pressure or temperature is applied to a fixed volume of gas, the gas temperature and pressure will change according to Gay-Lussac's law, which states that one megapascal pressure increase from room (atmospheric) pressure in a fixed volume of gas can raise the temperature up to 2697 degree Celsius theoretically. Accordingly, fixed volume gas pressure measurement does not satisfy equation (9) separation requirements. Nevertheless, the pressure measurement results become valid when the temperature returns back to the normal value when thermal equilibrium achieved. As the temperature will take time to return to ambient temperature, medium time delay will occur in the gas pressure measurement.

Time response of the pressure measurements was studied in my experiments. It was observed that when the medium under pressure is gas, it takes time for the pressure measurement to reach a stable value. Figure 37 illustrates measurement results at 4 temperature level for the gas medium as well as the measurement when the sensor was subjected to the water pressure. As shown in this figure, the pressure values do not change with time in liquid (water) because the temperature achieves equilibrium very quickly.

The time delay in the pressure measurement has a detrimental effect on FBG pressure sensor in practice and therefore, should be eliminated. By analyzing the data, I discovered that the wavelength shifts of two FBG's polarization peaks delay by the same amount of time,

consequently, the separation of the two polarization peaks responds to the pressure change instantaneously. In addition, the peak separation show excellent linearity at different temperature levels as it is shown in Figure 38. Temperature can be measured by tracking the peak movement in the same direction and therefore realizing the simultaneous measurement of pressure and temperature. 

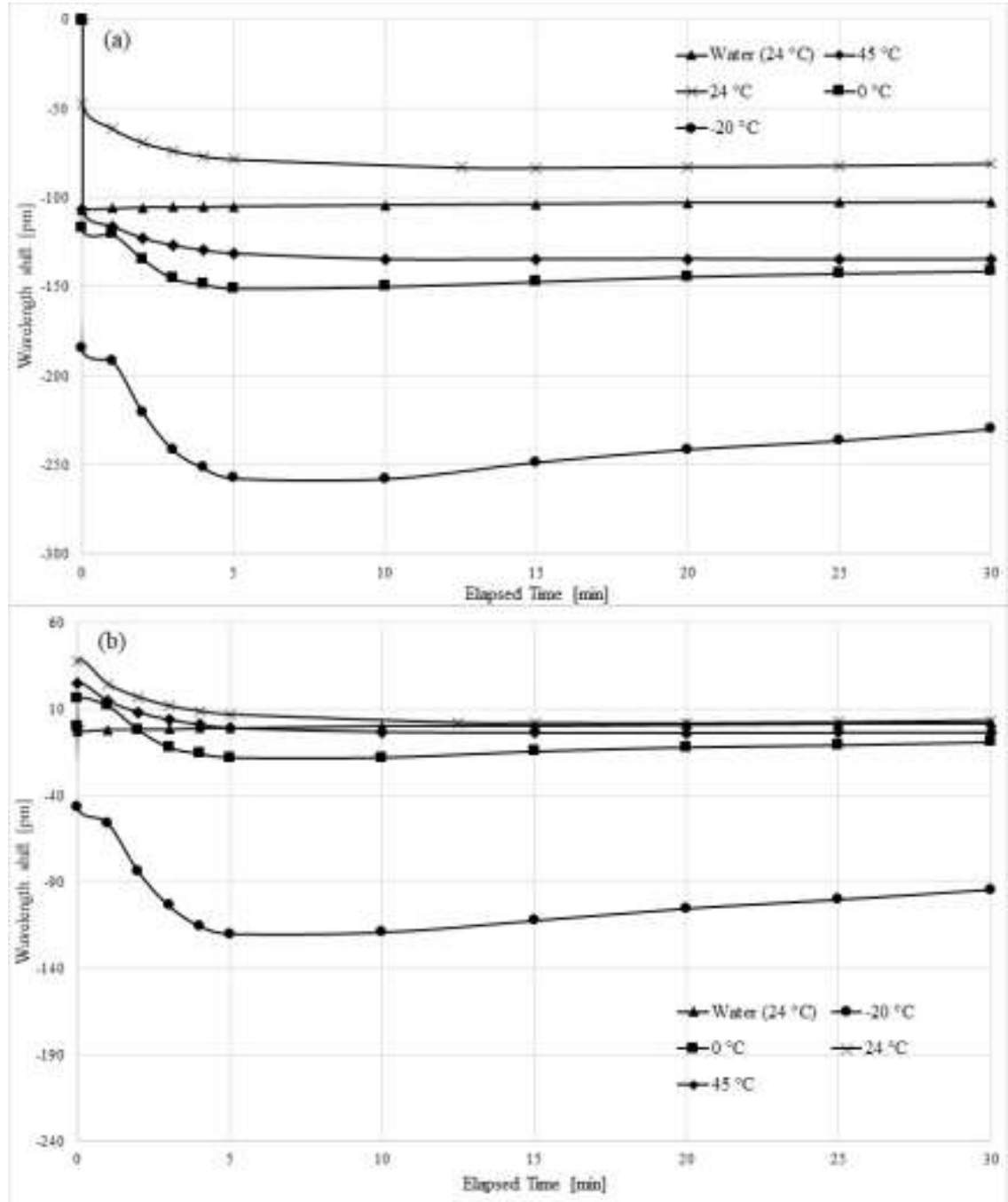

Figure 37 - time dependency of gas and water pressure measurement in different temperatures (a) fast axis (b) slow axis 

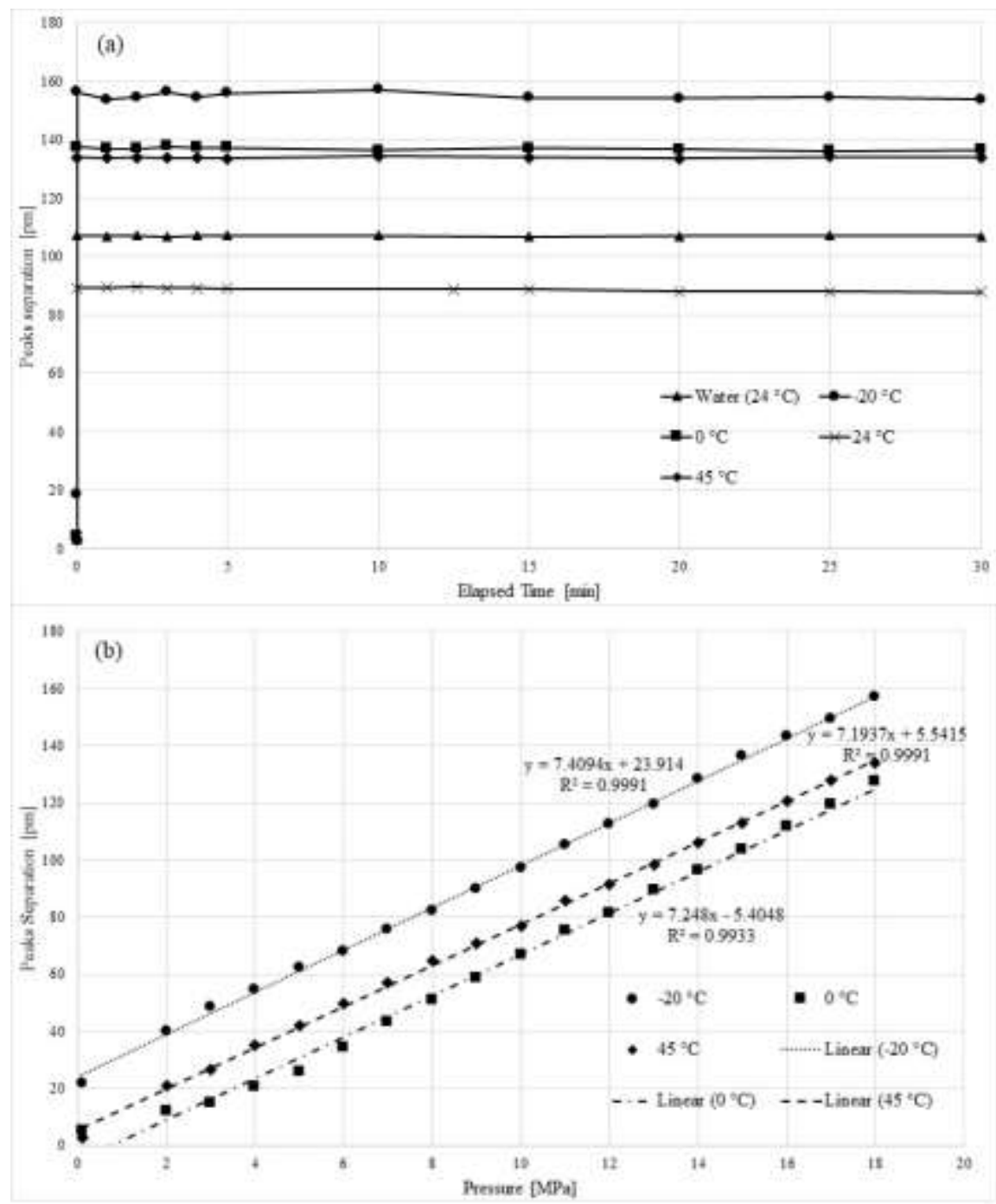

Figure 38 - peaks separation measurement method (a) time dependency (b) pressure measurement

\subsection{Pressure Measurement Versus Simulation}

The wavelength shifts of FBG's two polarization states and simulation result of FBG pressure measurement at room temperature are plotted in Figure 39. Measured values are linear with an $\mathrm{R}^{2}=0.9994$ and a slope of $-6.75 \mathrm{pm} / \mathrm{MPa}$ which is $13 \%$ higher than the calculated value for first (fast axis) peak. The results of the experiment are fairly close to the theoretical values for the second (slow axis) peak. 


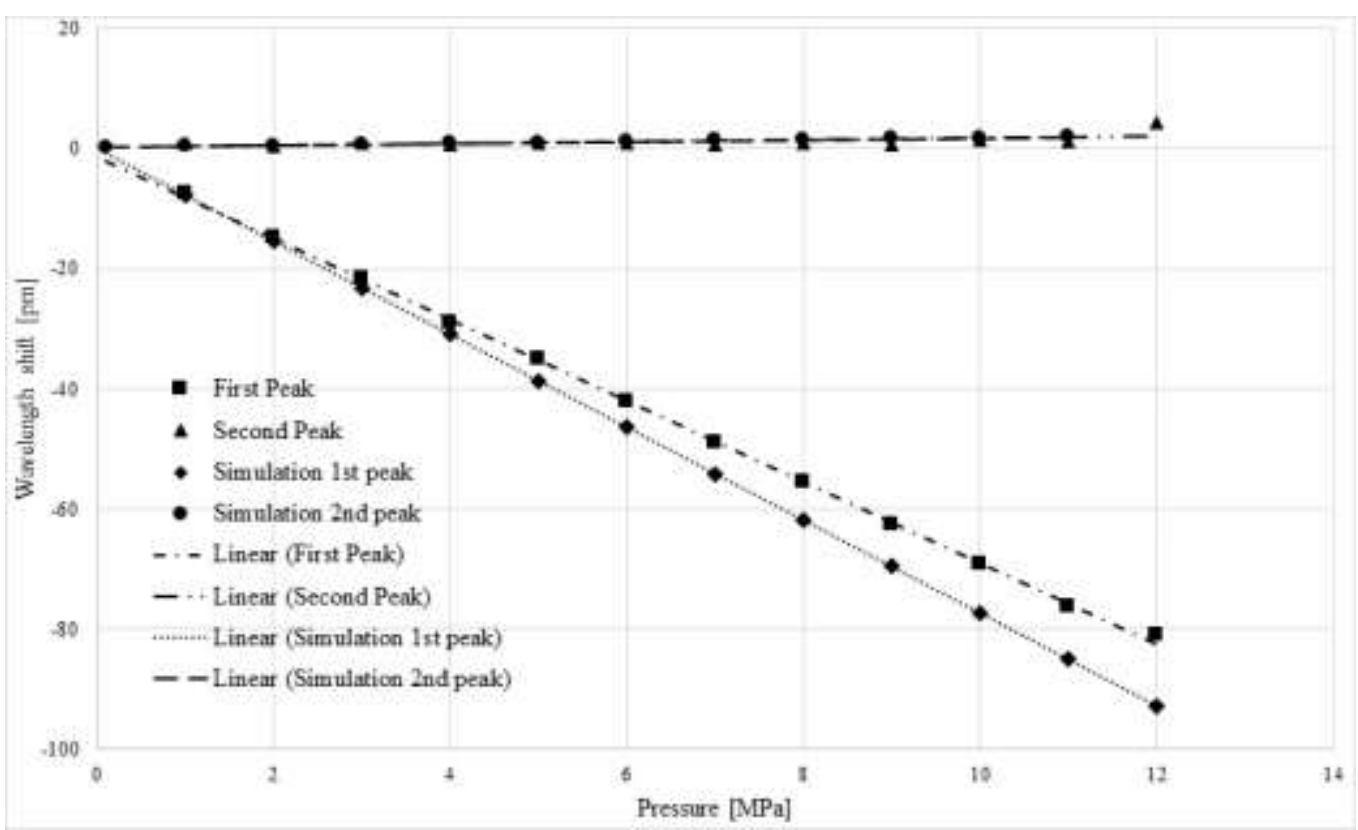

Figure 39 - FBG pressure measurement at room temperature simulation and experimental results

Theoretical simulations are always accompanied by certain errors since it is impossible to derive and solve exact algebraic and differential equations at all the points of a physical and/or time domain as it is discussed in previous sections. The errors come from geometrical simplification, model discretization, physical and mechanical properties inaccuracy, and assumptions in simulation/numerical solution of equations. Some of the sources of the error in the simulation part of this work are discussed here:

A. Physical and mechanical properties: It is difficult to obtain the exact value of mechanical constants such as Young's modulus and Poisson's ratio especially in the case of germanium doped silica as the core material. These parameters require specific experiments and expensive equipment to measure. For this reason, the parameters used in this study are obtained from literature which may be slightly different from their real values.

As another simplification, the effect of air locked inside the void in microstructured FBGs is not considered and the holes are simulated as a vacuum space. The air inside the bounded area of holes acts like air spring and may affect the behaviour of the FBG. However, as the holes are blocked at room pressure in reality, the air pressure inside them is the same as room pressure which is negligible compare with the hydrostatic pressure applied in the simulation. 
Another simplifications in this simulation, and also the most significant one, is the averaging procedure for the refractive index changing on wavelength shift which is broadly explained in previous sections. The refractive index changes due to the stress components in every single point of the FBG core. In addition to this, each node in fiber core has its own sinusoidal refractive index changes resulting from the FBG writing process. Combining these two effects together results in a very complicated mechanical and electromagnetic wave equations which should be solved simultaneously. Although some software like COMSOL can simulate this kind of multi-physics phenomena, an ordinary computer cannot handle the complexity of calculation.

The effect of coating is also neglected for the sake of simplification. Coating of the fiber makes it stiffer in reaction to the applied load and may change the stress distributions and strain values. This coating should be simulated in order to achieve more accurate results. Nevertheless, this requires a more powerful computer and consumes more time.

In addition to all of these, in a more complicated simulation for more precise results, the effect of bonding stress between core and cladding and cladding and coating as well as the flexibility of end support which connects fiber buffer to the pressure chamber can be considered in the modeling procedure.

B. Model discretization: Discretization of a simulated domain has a key effect on the results precision. Mesh elements with appropriate quality have a relatively same dimension in three directions. In fiber optics, the length is extremely higher than cross section dimensions which makes it difficult to create smooth elements. Though the results accuracy can be improved by increasing the number of mesh elements.

C. Geometrical simplification: The exact dimensions of the fiber cross section may be different from assumed size. The variation is a result of fabrication and measurement tolerances. 


\section{Conclusion and Future Works}

\subsection{Conclusion}

Pi-phase shifted FBGs made in microstructured fiber have been demonstrated successfully for measuring temperature and pressure simultaneously. There is no need of transducers for pressure or compensation for temperature measurement in this method which substantially reduces the weight and complexity of the sensor and sensing procedure. This type of sensors can eliminate the effect of temperature fluctuation on pressure measurement which is valuable in many applications. By the mean of holey fiber FBGs, I have achieved a temperature insensitive pressure sensor or a sensor for simultaneous measurement of pressure and temperature with a potential of implementation of monitoring multiple stations in one line using multiplexing technique. The FBG sensor can be installed in both gas media and liquid media and has the capability of eliminating the time delay in gas pressure measurement caused by the Gas law. This fast response attribute makes microstructured FBG pressure sensor appropriate for dynamic pressure measurement. The sensor is also extremely small, only a few $\mathrm{cm}$ in length and $0.3 \mathrm{~mm}$ in diameter that can be embedded in a very small tube, opening or chamber.

Further, the effects of cross sectional dimension such as core aspect ratio, holes diameter and their position in the fiber cross section as well as the configuration and installation method of FBG pressure sensors in different application have been studied theoretically to achieve best performance and higher sensitivity. Using demonstrated method, the optimum dimension and configuration of microstructured FBG can be obtained during a design procedure before the manufacturing and installation of sensor to achieve the highest possible sensitivity for each particular application.

\subsection{Future Works}

The simulation of FBG sensor suggested in this work could be assessed by more experiments with different types of fiber cross sections in order to validate the simulation as a viable solution for the development of specific FBG pressure sensors. In this way, the finite element analysis also should be improved by adding more geometric details in modelling, using more accurate mechanical parameters of material, and by combining the solid mechanic analysis with electromagnetic wave 
calculation inside the core of fiber. An ideal designing approach represents the required dimension of fiber cross section for a specific sensitivity.

Since the pressure can be measured instantaneously with this sensor, dynamic pressure studies can be another future work which could have important applications in jet engine research, oil and gas industry etc. 


\section{References}

[1] Micro-Measurements, "Design Considerations for Diaphragm Pressure Transducers", Strain Gages and Instruments, Tech Note TN-510-1, Nov-2010.

[2] James Karki, "Signal Conditioning Piezoelectric Sensors", Texas Instruments Application Report, SLOA033A - September 2000.

[3] "Introduction to General Piezoelectric Pressure Sensors", OPCB Piezotronics Inc.

[4] V. Sharapov, "Piezoceramic Sensors", Microtechnology and MEMS, DOI 10.1007/978-3-64215311-2 1, @ Springer-Verlag Berlin Heidelberg 2011.

[5] Alan D. Kersey et al., "Fiber Grating Sensors", Journal of lightwave technology, vol. 15, no. 8, August 1997.

[6] Chuang Wu, et al., "Characterization of Pressure Response of Bragg Gratings in Grapefruit Microstructured Fibers", Journal of lightwave technology, vol. 28, no. 9, may 1, 2010.

[7] Kevin P. Chen et al., "Regenerated gratings in air-hole microstructured fibers for hightemperature pressure sensing", Optics letters, vol. 36, no. 18 / september 15, 2011.

[8] Hwa-Yaw Tam et al., "Hydrostatic pressure sensor based on fiber Bragg grating written in single-ring suspended fiber", sixth European Workshop on Optical Fibre Sensors, edited by Elfed Lewis, Proc. of SPIE Vol. 9916, 99161R

[9] Sanne Sulejmani et al., "Control Over the Pressure Sensitivity of Bragg Grating-Based Sensors in Highly Birefringent Microstructured Optical Fibers", IEEE photonics technology letters, vol. 24, no. 6, march 15, 2012.

[10] David V. Hutton, "Fundamentals of Finite Element Analysis", The McGraw Hill Companies, 2004. 
[11] Andrew D. Yablon, "Optical and Mechanical Effects of Frozen-in Stresses and Strains in Optical Fibers", IEEE journal of selected topics in quantum electronics, vol. 10, no. 2, March/April 2004. 